\title{
Chiral Lagrangians for mesons with a single heavy quark
}

\author{
Shao-Zhou Jiang, ${ }^{1, *}$ Yan-Rui Liu, ${ }^{2, \dagger}$ and Qin-He Yang ${ }^{1}$ \\ ${ }^{1}$ Key Laboratory for Relativistic Astrophysics, Department of Physics, Guangxi University, \\ Nanning 530004, People's Republic of China \\ ${ }^{2}$ School of Physics, Shandong University, Jinan 250100, People's Republic of China
}

(Received 30 January 2019; published 16 April 2019)

\begin{abstract}
We construct the relativistic chiral Lagrangians for heavy-light mesons $(Q \bar{q})$ to the $\mathcal{O}\left(p^{4}\right)$ order. From $\mathcal{O}\left(p^{2}\right)$ to $\mathcal{O}\left(p^{4}\right)$, there are 17, 67, and 404 independent terms in the flavor $S U(2)$ case and 20, 84, and 655 independent terms in the flavor $S U(3)$ case. The Lagrangians in the heavy quark limit are also obtained. From $\mathcal{O}\left(p^{2}\right)$ to $\mathcal{O}\left(p^{4}\right)$, there are 7, 25, and 136 independent terms in the flavor $S U(2)$ case and 8, 33, and 212 independent terms in the flavor $S U(3)$ case. The relations between low-energy constants based on the heavy quark symmetry are also given up to the $\mathcal{O}\left(p^{3}\right)$ order.
\end{abstract}

DOI: 10.1103/PhysRevD.99.074018

\section{INTRODUCTION}

The spontaneous breaking of the global chiral symmetry of QCD is an important feature in the low-energy nonperturbative region of strong interactions. It has been widely accepted that the low-lying pseudoscalar mesons are those Goldstone bosons generated from the symmetry breaking. The effective theory based on this symmetry and its breaking is the chiral perturbation theory (ChPT) [1-3], which originally describes only low-energy dynamics of such mesons. Later, the theory was extended to cases involving octet baryons [4], decuplet baryons [5,6], and heavy quark hadrons $[7,8]$. The matter fields involved in the present study are those heavy-light mesons whose quark content is $Q \bar{q}(Q=c, b ; q=u, d, s)$.

Because of the light quark, the low-energy interactions for the heavy-light mesons are governed by the chiral symmetry. In addition, the interactions also obey the spinflavor heavy quark (HQ) symmetry in the limit $M_{Q} \rightarrow \infty$ [8-11]. The heavy quark flavor symmetry means that different heavy flavors have the same dynamics while the heavy quark spin symmetry results in degenerate hadron doublets containing states with different spins. In ChPT, increasing number of low-energy constants (LECs) need to be determined when high order chiral corrections are considered. For the case involving the heavy-light mesons, the heavy quark symmetry may provide relations

\footnotetext{
jjsz@gxu.edu.cn

yrliu@sdu.edu.cn
}

Published by the American Physical Society under the terms of the Creative Commons Attribution 4.0 International license. Further distribution of this work must maintain attribution to the author(s) and the published article's title, journal citation, and DOI. Funded by SCOAP. between LECs [12]. Before we can determine their values with other approaches, these constraints just from symmetry are certainly instructive. Of course, the corrections to such relations due to finite quark mass may also be needed for more detailed investigations.

With the chiral Lagrangians involving heavy-light mesons, a wide range of problems can be studied [13-25], such as properties of heavy-light mesons, mass difference between heavy-light mesons in the same doublet, interactions between the Goldstone bosons and the heavy-light mesons, interactions between heavy-light mesons, properties of new open-flavor particles [26], and so on. Up to now, the chiral Lagrangian in the sector of light pseudoscalar mesons has been constructed up to the $\mathcal{O}\left(p^{8}\right)$ order [27-35]. Recently, there have also been developments in the sector of light baryons [36-42]. However, the existing heavy-light meson chiral Lagrangian is still at low orders. The leading order result was obtained long time ago $[7,8,12]$. For higher order results, only parts of them were constructed for special problems, which can be found in Refs. [13-25]. Some similar works are about the $S U(2)$ pion-kaon chiral Lagrangian. This chiral Lagrangian has the same structures as that for the heavy-light pseudoscalar mesons. It has been constructed to the $\mathcal{O}\left(p^{4}\right)$ order [43-45]. In the present work, we systematically construct the relativistic chiral Lagrangians in the sector of heavylight mesons up to the fourth chiral order. To find some relations of LECs by using the heavy quark symmetry, we also construct directly chiral Lagrangians with the superfield $H$ containing the $J^{P}=0^{-}$and $1^{-} Q \bar{q}$ mesons. By comparing the relativistic Lagrangians with those in the HQ limit, one obtains relations between LECs.

This paper is organized as follows. In Sec. II, we review briefly the building blocks for the construction of chiral Lagrangians. In Sec. III, from the structures of Lagrangians 
to linear relations between various ingredients, we introduce the procedure to construct the heavy-light meson chiral Lagrangians step by step. In Sec. IV, the way to find relations between LECs with the heavy quark symmetry is introduced. In Sec. V, we list our results. The last Sec. VI is a short summary.

\section{DEFINITIONS AND BUILDING BLOCKS}

In this section, we give the building blocks necessary for the construction of Lagrangians. Some simple properties are also shown. These building blocks involve both relativistic and HQ forms. One may find details about them in Refs. [3,7,8,12,27,29,30,32,34,35,46-49].

\section{A. Goldstone bosons and external sources}

The QCD Lagrangian $\mathcal{L}_{\mathrm{QCD}}^{0}$ with $N_{f}$-flavor massless quarks is

$$
\mathcal{L}=\mathcal{L}_{\mathrm{QCD}}^{0}+\bar{q}\left(\not p+\not d \gamma_{5}-s+i p \gamma_{5}\right) q,
$$

where $q$ denotes the light quark field. $s, p, v^{\mu}$, and $a^{\mu}$ are scalar, pseudoscalar, vector, and axial-vector external sources, respectively. The tensor source and the $\theta$ term are ignored in this paper. As usual, both $a^{\mu}$ and $v^{\mu}$ are considered traceless in the flavor $S U(3)$ case, but only $a^{\mu}$ is traceless in the flavor $S U(2)$ case to study the electroweak interactions.

In ChPT, the low-lying pseudoscalar mesons are considered to be Goldstone bosons coming from the spontaneous breaking of the global symmetry $S U\left(N_{f}\right)_{L} \times$ $S U\left(N_{f}\right)_{R}$ into $S U\left(N_{f}\right)_{V}$. The meson field $u$ in matrix form transforms as

$$
u \rightarrow g_{R} u h^{\dagger}=h u g_{L}^{\dagger}
$$

under the chiral rotation, where $g_{L}$ and $g_{R}$ are group elements of $S U\left(N_{f}\right)_{L}$ and $S U\left(N_{f}\right)_{R}$, respectively, and $h$ is a compensator field which is a function of the pion fields.

Usually, the meson fields and external sources are combined to building blocks whose forms are as follows,

$$
\begin{aligned}
u^{\mu} & =i\left\{u^{\dagger}\left(\partial^{\mu}-i r^{\mu}\right) u-u\left(\partial^{\mu}-i l^{\mu}\right) u^{\dagger}\right\}, \\
\chi_{ \pm} & =u^{\dagger} \chi u^{\dagger} \pm u \chi^{\dagger} u, \\
h^{\mu \nu} & =\nabla^{\mu} u^{\nu}+\nabla^{\nu} u^{\mu}, \\
f_{+}^{\mu \nu} & =u F_{L}^{\mu \nu} u^{\dagger}+u^{\dagger} F_{R}^{\mu \nu} u, \\
f_{-}^{\mu \nu} & =u F_{L}^{\mu \nu} u^{\dagger}-u^{\dagger} F_{R}^{\mu \nu} u=-\nabla^{\mu} u^{\nu}+\nabla^{\nu} u^{\mu},
\end{aligned}
$$

where $r^{\mu}=v^{\mu}+a^{\mu}, \quad l^{\mu}=v^{\mu}-a^{\mu}, \quad \chi=2 B_{0}(s+i p)$, $F_{R}^{\mu \nu}=\partial^{\mu} r^{\nu}-\partial^{\nu} r^{\mu}-i\left[r^{\mu}, r^{\nu}\right], F_{L}^{\mu \nu}=\partial^{\mu} l^{\nu}-\partial^{\nu} l^{\mu}-i\left[l^{\mu}, l^{\nu}\right]$, and $B_{0}$ is a constant related to the quark condensate. The covariant derivative for any building block $X$ is defined through

$$
\begin{gathered}
\nabla^{\mu} X=\partial^{\mu} X+\left[\Gamma^{\mu}, X\right] \\
\Gamma^{\mu}=\frac{1}{2}\left\{u^{\dagger}\left(\partial^{\mu}-i r^{\mu}\right) u+u\left(\partial^{\mu}-i l^{\mu}\right) u^{\dagger}\right\} .
\end{gathered}
$$

The advantage of using these building blocks is that all of them transform under the chiral rotation $(R)$ in the same way,

$$
X \stackrel{R}{\longrightarrow} X^{\prime}=h X h^{\dagger} .
$$

\section{B. Heavy-light mesons}

A heavy-light meson contains one heavy quark $Q$ ( $c$ or $b)$ and one light antiquark $\bar{q}(\bar{u}, \bar{d}$, or $\bar{s})$. The lowest lying heavy-light mesons are the pseudoscalar $P$ with $J^{P}=0^{-}$ and vector $P^{*}$ with $J^{P}=1^{-}$. In the flavor $S U(3)$ case ( $Q$ as a flavor singlet), they are represented as row vectors,

$P=\left\{\begin{array}{l}\left(D^{0}, D^{+}, D_{s}^{+}\right), \\ \left(\bar{B}^{-}, \bar{B}^{0}, \bar{B}_{s}^{0}\right),\end{array} \quad P^{*}=\left\{\begin{array}{l}\left(D^{* 0}, D^{*+}, D_{s}^{*+}\right), \\ \left(\bar{B}^{*-}, \bar{B}^{* 0}, \bar{B}_{s}^{* 0}\right) .\end{array}\right.\right.$

In the flavor $S U(2)$ case, the third $Q \bar{s}$ mesons need to be removed. The Lagrangians in these two cases have different independent chiral-invariant terms and we consider results in both cases. The covariant derivative for $\tilde{P}\left(P\right.$ or $\left.P^{* \mu}\right)$ is

$$
\begin{aligned}
D^{\mu} \tilde{P}^{\dagger} & =\left(\partial^{\mu}+\Gamma^{\mu}\right) \tilde{P}^{\dagger}, \\
D^{\mu} \tilde{P} & =\tilde{P}\left(\overleftarrow{\partial}^{\mu}+\Gamma^{\mu \dagger}\right),
\end{aligned}
$$

$D^{\mu \nu \cdots \rho} \equiv \frac{1}{n !}(\underbrace{D^{\mu} D^{\nu} \cdots D^{\rho}}_{n}+$ full permutation of $D^{\prime} \mathrm{s})$.

Equation (10) defines a totally symmetrical covariant derivative like the $\pi N$ case [36]. The reason for this definition is that permutations of derivatives acting on a building block do not change the chiral dimension (as Eq. (23) below). The defined symmetrical derivative will simplify some calculations (see Sec. IV). The chiral transformations for heavy-light meson fields read

$$
\tilde{P} \stackrel{R}{\longrightarrow} \tilde{P}^{\prime}=\tilde{P} h^{\dagger}, \quad \tilde{P}^{\dagger} \stackrel{R}{\longrightarrow} \tilde{P}^{\prime \dagger}=h \tilde{P}^{\dagger} .
$$

To adopt the heavy quark symmetry, one collects the heavy-light mesons in a superfield as usual [50],

$H=\sqrt{M} \frac{1+\not p}{2}\left(P_{Q, \mu}^{*} \gamma^{\mu}+\delta P_{Q} \gamma_{5}\right), \quad \bar{H} \equiv \gamma^{0} H^{\dagger} \gamma^{0}$,

where $M \equiv M_{P}=M_{P^{*}}$ is the heavy-light meson masses in the HQ limit and $v^{\mu}$ with $v^{2}=1$ is the velocity of heavylight mesons, $P_{Q, \mu}^{*}$ and $P_{Q}$ only contain the annihilation operator. Now, $H$ contains only annihilation operators for 
$Q \bar{q}$ mesons and its mass dimension is $3 / 2$. We here use $\delta$ to denote the arbitrary relative phase between the mesons $P_{Q}$ and $P_{Q}^{*}$. Different conventions exist in the literature, e.g., $\delta=1$ in [7], $\delta=-1$ in [47], and $\delta=i$ in [51]. This phase does not have physical effects and its choice does not impact on the form of Lagrangians, either. Scaling the superfield by $e^{-i M v \cdot x}$ will modify the energy measure from $M_{Q}$ to $M$ and the covariant derivative on matter fields becomes $D^{\mu} H(x)=-i M v^{\mu} H(x)$. Obviously, the chiral transformations for $H$ and $\bar{H}$ are the same as those for $\tilde{P}$ and $\tilde{P}^{\dagger}$, respectively.

\section{LAGRANGIAN CONSTRUCTION}

This section shows the basic steps to construct the Lagrangian for heavy-light mesons. First, one analyzes the structures of chiral Lagrangians because they have effects on some properties of building blocks. Second, one establishes the $P$-parity, $C$-parity, and Hermitian properties of all the building blocks. Third, one finds out available linear relations in order to reduce linearly dependent terms. Finally, one constructs all possible structures of the chiral Lagrangian and gets independent terms by using the linear relations.

\section{A. Structures of chiral Lagrangians}

The relativistic heavy-light meson chiral Lagrangian can be written as

$$
\begin{gathered}
\mathcal{L}=\mathcal{L}_{P P}+\mathcal{L}_{P^{*} P^{*}}+\mathcal{L}_{P P^{*}} \\
=\sum_{n} C_{n} P \cdots P^{\dagger}+\sum_{m} C_{m} P^{*} \cdots P^{* \dagger} \\
+\sum_{p} C_{p}\left(P \cdots P^{* \dagger}+P^{*} \cdots P^{\dagger}\right),
\end{gathered}
$$

where $\mathcal{L}_{P P}, \mathcal{L}_{P^{*} P^{*}}$, and $\mathcal{L}_{P P^{*}}$ represent the interaction terms involving only heavy pseudoscalar mesons, only heavy vector mesons, and both heavy pseudoscalar and heavy vector mesons, respectively. The symbol "..." includes allowed combinations of building blocks given in Sec. II A and appropriate coefficients $( \pm 1$ or $\pm i$ ) to keep the symmetry of $\mathcal{L}$. For convenience, the LECs $\left(C_{n}, C_{m}\right.$, and $C_{p}$ ) are all assumed to be real constants and we use the convention that all the possible covariant derivatives in "..." act on the right side heavy-light meson fields.

To find out relations between LECs in the HQ limit, we also construct chiral Lagrangians involving the superfield $H$ directly. The Lagrangian in this formalism looks like

$$
\mathcal{L}=\sum_{n} D_{n}\langle H \cdots \Gamma \bar{H}\rangle,
$$

where $D_{n}$ 's represent LECs in this case, $\Gamma$ is an element of the Clifford algebra, and $\langle\cdots\rangle$ means trace in the spin
TABLE I. Chiral dimension (Dim), parity $(P)$, charge conjugation $(C)$, and Hermiticity (H.c.) of the building blocks, the matter fields, and the Levi-Civita tensor.

\begin{tabular}{lcccc}
\hline \hline & Dim & $P$ & $C$ & H.c. \\
\hline$u^{\mu}$ & 1 & $-u_{\mu}$ & $\left(u^{\mu}\right)^{T}$ & $u^{\mu}$ \\
$h^{\mu \nu}$ & 2 & $-h_{\mu \nu}$ & $\left(h^{\mu \nu}\right)^{T}$ & $h^{\mu \nu}$ \\
$\chi_{ \pm}$ & 2 & $\pm \chi_{ \pm}$ & $\left(\chi_{ \pm}\right)^{T}$ & $\pm \chi_{ \pm}$ \\
$f_{ \pm}^{\mu \nu}$ & 2 & $\pm f_{ \pm \mu \nu}$ & $\mp\left(f_{ \pm}^{\mu \nu}\right)^{T}$ & $f_{ \pm}^{\mu \nu}$ \\
$P$ & 0 & $-P$ & $\left(P^{\dagger}\right)^{T}$ & $P^{\dagger}$ \\
$P^{* \mu}$ & 0 & $P_{\mu}^{*}$ & $\left(P^{* \mu^{\dagger}}\right)^{T}$ & $P^{* \mu^{\dagger}}$ \\
$D^{\mu} P$ & 0 & $-D_{\mu} P$ & $\left(D^{\mu} P^{\dagger}\right)^{T}$ & $\left(D^{\mu} P\right)^{\dagger}$ \\
$D^{\mu} P^{* \nu}$ & 0 & $D_{\mu} P_{\nu}^{*}$ & $\left(D^{\mu} P^{* \nu \dagger}\right)^{T}$ & $\left(D^{\mu} P^{* \nu}\right)^{\dagger}$ \\
$\varepsilon^{\mu \nu \lambda \rho}$ & 0 & $-\varepsilon_{\mu \nu \lambda \rho}$ & $\varepsilon^{\mu \nu \lambda \rho}$ & $\varepsilon^{\mu \nu \lambda \rho}$ \\
\hline \hline
\end{tabular}

space. If flavor traces for building blocks are needed in “...", we also use this symbol $\langle\cdots\rangle$. The heavy quark symmetry requires that the position of $\Gamma$ should be after $H$ but before $\bar{H}$.

\section{B. Properties of building blocks}

The properties of the building blocks have been discussed in a lot of references. Here we only collect relevant results. One may find details about them in Refs. [2,3,7,27,29-31,34-36,47,51].

Table I lists the chiral dimensions, parity transformations $(P)$, charge conjugation transformations $(C)$, and Hermitian transformations (H.c.) of the building blocks, the matter fields, and the Levi-Civita tensor. Since the heavy-light mesons are not purely neutral states, the phases for the charge conjugation transformation of them are uncertain. Choosing " + " for $P$ is natural since $J^{P C}=0^{--}$are exotic quantum numbers. For $P^{*}$, we use the convention " + " and will discuss another one.

Table II lists the corresponding properties of the Clifford algebra and the velocity of heavy-light mesons, which are considered between $H$ and $\bar{H}$ as (15). $H, \bar{H}$, and $v^{\mu}$ are chiral dimensionless and their properties are considered together with Clifford algebra, like the $\pi N$ case in Ref. [36]. Table II only displays the extra signs. We do not show anything about $\gamma_{5}$ because $\left\langle H \gamma_{5} \bar{H}\right\rangle=0$ gives no contributions. $H$ only contains the $Q \bar{q}$ fields, but not $\bar{Q} q$ fields. Hence the Lagrangian in heavy quark symmetry does not

TABLE II. Chiral dimension (Dim), parity $(P)$, charge conjugation $(C)$, and Hermiticity (H.c.) of the Clifford algebra elements and the velocity of heavy-light mesons.

\begin{tabular}{lcccc}
\hline \hline & Dim & $P$ & $C$ & H.c. \\
\hline 1 & 0 & + & + & + \\
$\gamma^{\mu}$ & 0 & + & - & + \\
$\gamma_{5} \gamma^{\mu}$ & 0 & - & + & + \\
$\sigma^{\mu \nu}$ & 0 & + & - & + \\
$v^{\mu}$ & 0 & + & - & + \\
\hline \hline
\end{tabular}


have to be $C$-invariant. The meaning of the charge conjugation in Table II will be discussed in Sec. IV.

\section{Linear relations}

Linear relations exist which are essential in reducing the chiral-invariant terms to a minimal set. For details about them, one may consult Refs. [27,29,30,34,35].

(i) Partial integration.

Ignoring higher order terms, one has

$$
0 \doteq \tilde{P} \check{L}^{\mu} X \tilde{P}^{\dagger}+\tilde{P} X D^{\mu} \tilde{P}^{\dagger}
$$

where $X$ is any building block or their products and ".." means that both sides are approximately equal with their difference appearing at the order $\mathcal{O}\left(p^{1}\right)$. With this relation, we can move the covariant derivatives to the right position so that they act only on the field $\tilde{P}^{\dagger}$.

(ii) Schouten identity.

This is a relation about the Levi-Civita tensor,

$$
\begin{gathered}
\epsilon^{\mu \nu \lambda \rho} A^{\sigma}-\epsilon^{\sigma \nu \lambda \rho} A^{\mu}-\epsilon^{\mu \sigma \lambda \rho} A^{\nu}-\epsilon^{\mu \nu \sigma \rho} A^{\lambda} \\
-\epsilon^{\mu \nu \lambda \sigma} A^{\rho}=0,
\end{gathered}
$$

where $A$ is anything having Lorentz index (indices). The five indices in the left-hand side are totally antisymmetric.

(iii) Equations of motions (EOMs).

The EOMs and subsidiary condition for light pseudoscalar and heavy-light mesons are

$$
\begin{gathered}
\nabla_{\mu} u^{\mu} \doteq \frac{i}{2}\left(\chi_{-}-\frac{1}{N_{f}}\left\langle\chi_{-}\right\rangle\right), \\
\left(D^{2}+M_{P}^{2}\right) P^{\dagger} \doteq 0 \\
\left(D^{2}+M_{P^{*}}^{2}\right) P_{\mu}^{* \dagger} \doteq 0 \\
D^{\mu} P_{\mu}^{* \dagger} \doteq 0 \\
v^{\mu} P_{Q, \mu}^{* \dagger}=0
\end{gathered}
$$

where $N_{f}$ is the number of light quark flavors and the conjugations of these equations are omitted. Equation (22) only works in the heavy quark limit. The right-hand sides of Eqs. (19)-(21) are at least at the order $\mathcal{O}\left(p^{1}\right)$. At the $\mathcal{O}\left(p^{1}\right)$ order, they contain one $u^{\mu}$. Hence, $D^{2} \tilde{P}$ can be changed to $-M_{\tilde{p}}^{2} \tilde{P}$ and does not happen in the Lagrangian. $D^{\mu} P_{\mu}^{*}$ is at the order $\mathcal{O}\left(p^{1}\right)$. It removes the redundant degree of freedom of $P_{\mu}^{*}$ field, and $D^{\mu} P_{\mu}^{*}$ does not appear in the Lagrangian, either. (iv) Covariant derivatives and Bianchi identity.

The commutative relation for the covariant derivatives acting on any building block $X$ is

$$
\begin{gathered}
{\left[\nabla^{\mu}, \nabla^{\nu}\right] X=\left[\Gamma^{\mu \nu}, X\right],} \\
\Gamma^{\mu \nu}=\frac{1}{4}\left[u^{\mu}, u^{\nu}\right]-\frac{i}{2} f_{+}^{\mu \nu} .
\end{gathered}
$$

Rewriting it explicitly, one has

$$
\begin{gathered}
\nabla^{\mu} \nabla^{\nu} X-\nabla^{\nu} \nabla^{\mu} X-\frac{1}{4} u^{\mu} u^{\nu} X+\frac{1}{4} u^{\nu} u^{\mu} X+\frac{i}{2} f_{+}^{\mu \nu} X \\
+\frac{1}{4} X u^{\mu} u^{\nu}-\frac{1}{4} X u^{\nu} u^{\mu}-\frac{i}{2} X f_{+}^{\mu \nu}=0 .
\end{gathered}
$$

Another relation about covariant derivatives is Bianchi identity

$$
\nabla^{\mu} \Gamma^{\nu \lambda}+\nabla^{\nu} \Gamma^{\lambda \mu}+\nabla^{\lambda} \Gamma^{\mu \nu}=0 .
$$

Its explicit form is

$$
\begin{gathered}
\nabla^{\mu} f_{+}^{\nu \lambda}+\nabla^{\nu} f_{+}^{\lambda \mu}+\nabla^{\lambda} f_{+}^{\mu \nu}+\frac{i}{2}\left[u^{\mu}, f_{-}^{\nu \lambda}\right] \\
+\frac{i}{2}\left[u^{\nu}, f_{-}^{\lambda \mu}\right]+\frac{i}{2}\left[u^{\lambda}, f_{-}^{\mu \nu}\right]=0 .
\end{gathered}
$$

These two explicit relations are for determining the strict relations of LECs which will be discussed in Sec. IV.

(v) Cayley-Hamilton relations.

Any $2 \times 2$ matrices $A$ and $B$ have the relation

$$
A B+B A-A\langle B\rangle-B\langle A\rangle-\langle A B\rangle+\langle A\rangle\langle B\rangle=0 .
$$

Any $3 \times 3$ matrices $A, B$, and $C$ have the relation

$$
\begin{aligned}
0= & A B C+A C B+B A C+B C A+C A B+C B A \\
& -A B\langle C\rangle-A C\langle B\rangle-B A\langle C\rangle-B C\langle A\rangle-C A\langle B\rangle \\
& -C B\langle A\rangle-A\langle B C\rangle-B\langle A C\rangle-C\langle A B\rangle-\langle A B C\rangle \\
& -\langle A C B\rangle+A\langle B\rangle\langle C\rangle+B\langle A\rangle\langle C\rangle+C\langle A\rangle\langle B\rangle \\
& +\langle A\rangle\langle B C\rangle+\langle B\rangle\langle A C\rangle+\langle C\rangle\langle A B\rangle-\langle A\rangle\langle B\rangle\langle C\rangle .
\end{aligned}
$$

(vi) Contact terms.

The contact terms need to be picked up independently. Such terms appear only at the $\mathcal{O}\left(p^{4}\right)$ order. To show their irrelevance with pion fields, we change the building blocks from $u^{\mu}, h^{\mu \nu}, f_{ \pm}^{\mu \nu}$, and $\chi_{ \pm}$ to $F_{R, L}^{\mu \nu}, \chi$, and $\chi^{\dagger}$. The relevant relations are 
TABLE III. Chiral rotations (R), parity $(P)$, charge conjugation $(C)$, and Hermiticity (H.c.) of the LR-basis.

\begin{tabular}{lcccc}
\hline \hline & $R$ & $P$ & $C$ & H.c. \\
\hline$\chi$ & $g_{R} \chi g_{L}^{\dagger}$ & $\chi^{\dagger}$ & $\chi^{T}$ & $\chi^{\dagger}$ \\
$\chi^{\dagger}$ & $g_{L} \chi^{\dagger} g_{R}^{\dagger}$ & $\chi$ & $\chi^{\dagger T}$ & $\chi$ \\
$F_{L}^{\mu \nu}$ & $g_{L} F_{L}^{\mu \nu} g_{L}^{\dagger}$ & $F_{R}^{\mu \nu}$ & $-\left(F_{R}^{\mu \nu}\right)^{T}$ & $F_{L}^{\mu \nu}$ \\
$F_{R}^{\mu \nu}$ & $g_{R} F_{R}^{\mu \nu} g_{R}^{\dagger}$ & $F_{L}^{\mu \nu}$ & $-\left(F_{L}^{\mu \nu}\right)^{T}$ & $F_{R}^{\mu \nu}$ \\
\hline \hline
\end{tabular}

$$
\begin{aligned}
F_{L}^{\mu \nu} & =\frac{1}{2} u^{\dagger}\left(f_{+}^{\mu \nu}+f_{-}^{\mu \nu}\right) u, \\
F_{R}^{\mu \nu} & =\frac{1}{2} u\left(f_{+}^{\mu \nu}-f_{-}^{\mu \nu}\right) u^{\dagger}, \\
\chi & =\frac{1}{2} u\left(\chi_{+}+\chi_{-}\right) u, \\
\chi^{\dagger} & =\frac{1}{2} u^{\dagger}\left(\chi_{+}-\chi_{-}\right) u^{\dagger} .
\end{aligned}
$$

We show the properties of these building blocks (LR-basis) [27,34] in Table III. The number of resultant contact terms is found small. They are listed at the end of each part for $\mathcal{L}_{P P}, \mathcal{L}_{P^{*} P^{*}}$, and $\mathcal{L}_{P P^{*}}$ in Table IX and such terms in the HQ limit are given at the end of Table $\mathrm{X}$.

The process to pick up independent terms is very boring and is done by computer. The details about the operation have been presented in Refs. [34,39,40].

\section{LEC RELATIONS IN THE HEAVY QUARK LIMIT}

According to the heavy quark symmetry, relations exist among LECs for $P P^{\dagger}$ terms, those for $P^{*} P^{* \dagger}$ terms, and those for $P P^{* \dagger}$ terms. In order to find them, we firstly redefine the independent terms and their corresponding LECs in Eq. (14) to be

$$
\tilde{O}_{n}=O_{n} / M^{r}, \quad \tilde{C}_{n}=C_{n} M^{r},
$$

where $r$ is the number of covariant derivative acting on the heavy-light meson fields. Now, all $\tilde{C}_{n}$ 's at a given order have the same mass dimension.

At least two methods can be used to get the LEC relations. The first one is to change the relativistic Lagrangians to the HQ form. With Eq. (12), one obtains

$$
\begin{array}{rlrl}
\sqrt{M} P_{Q} & =\frac{1}{2} \delta^{*}\left\langle H \gamma_{5}\right\rangle, & \sqrt{M} P_{Q, \mu}^{*}=\frac{1}{2}\left\langle H \gamma_{\mu}\right\rangle, \\
\sqrt{M} P_{Q}^{\dagger}=-\frac{1}{2} \delta\left\langle\bar{H} \gamma_{5}\right\rangle, & \sqrt{M} P_{Q, \mu}^{* \dagger}=\frac{1}{2}\left\langle\bar{H} \gamma_{\mu}\right\rangle .
\end{array}
$$

These fields contain only operators to annihilate or generate $Q \bar{q}$ mesons, but no operators for $\bar{Q} q$ mesons. If we assume that the fields in Eq. (14) also describe only $Q \bar{q}$ mesons, the Lagrangian there can be changed to that in Eq. (15) by using the Fierz identity. Retaining only terms satisfying the heavy quark symmetry and comparing independent terms, one can obtain relations between $C_{n}$ 's and $D_{n}$ 's.

The second method is opposite by changing the form of Eq. (15) to that of Eq. (14),

$$
\begin{gathered}
\langle H \bar{H}\rangle \rightarrow M\left(-2 P_{Q} P_{Q}^{\dagger}+2 P_{Q}^{* \mu} P_{Q, \mu}^{* \dagger}\right), \\
\left\langle H \gamma_{5} \bar{H}\right\rangle \rightarrow 0, \\
\left\langle H \gamma^{\mu} \bar{H}\right\rangle \rightarrow M\left(-\left\langle H v^{\mu} \bar{H}\right\rangle\right)=\sqrt{M}\left(-2 i P_{Q} D^{\mu} P_{Q}^{\dagger} / M\right. \\
\left.+2 i P_{Q}^{* \nu} D^{\mu} P_{Q, \nu}^{* \dagger} / M\right), \\
\left\langle H \gamma_{5} \gamma^{\mu} \bar{H}\right\rangle \rightarrow M\left(-2 \varepsilon^{\mu \nu \lambda \rho} P_{Q, \lambda}^{*} D_{\nu} P_{Q, \rho}^{* \dagger} / M+2 \delta P_{Q} P_{Q}^{* \dagger \mu}\right. \\
\left.+2 \delta^{*} P_{Q}^{* \mu} P_{Q}^{\dagger}\right), \\
\left\langle H \sigma^{\mu \nu} \bar{H}\right\rangle \rightarrow M\left(2 i P_{Q}^{* \mu} P_{Q}^{* \dagger \nu}-2 i P_{Q}^{* \nu} P_{Q}^{* \dagger \mu}\right. \\
+2 i \varepsilon^{\mu \nu \lambda \rho} \delta P_{Q} D_{\lambda} P_{Q, \rho}^{* \dagger} / M \\
\left.+2 i \varepsilon^{\mu \nu \lambda \rho} \delta^{*} P_{Q, \rho}^{*} D_{\lambda} P_{Q}^{\dagger} / M\right),
\end{gathered}
$$

where we have used the definition $\left\langle\gamma^{\mu} \gamma^{\nu} \gamma^{\lambda} \gamma^{\rho} \gamma_{5}\right\rangle=-4 i \varepsilon^{\mu \nu \lambda \rho}$. The factor $M$ comes from the definition in Eq. (12). From the above equations, one finds that only structures $\langle H \bar{H}\rangle$, $\left\langle H \gamma_{5} \gamma^{\mu} \bar{H}\right\rangle$, and $\left\langle H \sigma^{\mu \nu} \bar{H}\right\rangle$ exist in the final results, a feature consistent with the pion-nucleon case [36]. In order to obtain the relativistic Lagrangian, the right-hand sides of the above equations also need $C$ invariant. If one substitutes $\tilde{P}_{Q} \rightarrow \tilde{P}$ and chooses the " $C$-parity" of the Clifford algebra and the velocity as those in Table II, these terms automatically contain the $C$-invariant.

To get the exact relations between $\tilde{C}_{k}$ and $D_{l}$, the strict linear relations are needed. In Sec. III C, we have found them in the relativistic case. Hence, we choose the second method to do the calculation. This method also avoids complex calculation from the Fierz identity. The relations are

$$
\tilde{C}_{k}=M \sum_{l} D_{l} A_{l k}
$$

where $M$ is a usual normalization factor coming from Eq. (12). All elements in matrix $A_{l k}$ are dimensionless. Since the number of $D_{l}$ is much less than the number of $\tilde{C}_{k}$ (see the results in Sec. V), $D_{l}$ may be obtained more easily in other ways. If all $D_{l}$ are known, Eq. (41) gives a rough estimation of $\tilde{C}_{k}$. It also gives some constraint conditions of $\tilde{C}_{k}$ in the heavy quark limit.

To calculate Eq. (41), we avoid the approximate relations (marked by "=") in Sec. III C as far as possible. Higher order contribution of the EOM for pseudoscalar mesons 
[Eq. (18)] does not work to the $\mathcal{O}\left(p^{2}\right)$ order, and higher order contribution of the EOMs for heavy-light mesons does not work to the $\mathcal{O}\left(p^{3}\right)$ order. Hence, all relations in Sec. III $C$ are strict ones to the $\mathcal{O}\left(p^{3}\right)$ order.

\section{RESULTS}

Following the above steps, we get the final results expressed as

$$
\begin{gathered}
\mathcal{L}^{(m)}=\sum_{n} C_{n}^{(m)} O_{n}^{(m)}=\sum_{n} \tilde{C}_{n}^{(m)} \tilde{O}_{n}^{(m)}, \quad N_{f}=3 \\
\mathcal{L}^{(m)}=\sum_{n} c_{n}^{(m)} o_{n}^{(m)}=\sum_{n} \tilde{c}_{n}^{(m)} \tilde{o}_{n}^{(m)}, \quad N_{f}=2 \\
\mathcal{L}_{\mathrm{H} Q}^{(m)}=\sum_{n} D_{n}^{(m)} P_{n}^{(m)}, \quad N_{f}=3 \\
\mathcal{L}_{\mathrm{H} Q}^{(m)}=\sum_{n} d_{n}^{(m)} p_{n}^{(m)}, \quad N_{f}=2 .
\end{gathered}
$$

where $m$ is the chiral dimension.

\section{A. Results at the $\mathcal{O}\left(p^{1}\right)$ and $\mathcal{O}\left(p^{2}\right)$ orders}

The obtained relativistic result at the leading order,

$$
\begin{aligned}
\mathcal{L}^{(1)}= & D_{\mu} P D^{\mu} P^{\dagger}-M_{P}^{2} P P^{\dagger} \\
& -\frac{1}{2}\left(D^{\mu} P^{* \nu}-D^{\nu} P^{* \mu}\right)\left(D_{\mu} P_{\nu}^{* \dagger}-D_{\nu} P_{\mu}^{* \dagger}\right)+M_{P^{*}}^{2} P^{* \mu} P_{\mu}^{* \dagger} \\
& +\frac{1}{2} f_{Q}\left(P u^{\mu} P^{* \dagger}{ }_{\mu}+\text { H.c. }\right) \\
& +\frac{1}{4} g_{Q} \varepsilon^{\mu \nu \lambda \rho}\left(P_{\rho}^{*} u_{\lambda}\left(D_{\mu} P_{\nu}^{* \dagger}-D_{\nu} P_{\mu}^{* \dagger}\right)+\text { H.c. }\right)
\end{aligned}
$$

is the same as that in Ref. [7]. The form obeying the heavy quark symmetry is [8]

$$
\mathcal{L}_{\mathrm{H} Q}^{(1)}=\left\langle H i v^{\mu} D_{\mu} \bar{H}\right\rangle-\frac{1}{2} g\left\langle H u_{\lambda} \gamma_{5} \gamma^{\lambda} \bar{H}\right\rangle .
$$

\begin{tabular}{|c|c|c|c|c|c|c|}
\hline$O_{n}$ & $S U(2)$ & $\tilde{c}_{n}^{(2)}$ & $\tilde{c}_{n}^{(2)}$ & $S U(3)$ & $\tilde{C}_{n}^{(2)}$ & $\tilde{C}_{n}^{(2)}$ \\
\hline$P u^{\mu} u_{\mu} P^{\dagger}$ & 1 & $-2 d_{1}^{(2)}$ & I & 1 & $-2 D_{1}^{(2)}$ & I \\
\hline$P u^{\mu} u^{\nu} D_{\mu \nu} P^{\dagger}$ & 2 & $2 d_{2}^{(2)}$ & I & 2 & $2 D_{2}^{(2)}$ & I \\
\hline$P\left\langle u^{\mu} u_{\mu}\right\rangle P^{\dagger}$ & & & & 3 & $-2 D_{4}^{(2)}$ & I \\
\hline$P\left\langle u^{\mu} u^{\nu}\right\rangle D_{\mu \nu} P^{\dagger}$ & & & & 4 & $2 D_{5}^{(2)}$ & I \\
\hline$P \chi_{+} P^{\dagger}$ & 3 & $-2 d_{6}^{(2)}$ & I & 5 & $-2 D_{7}^{(2)}$ & I \\
\hline$P\left\langle\chi_{+}\right\rangle P^{\dagger}$ & 4 & $-2 d_{7}^{(2)}$ & I & 6 & $-2 D_{8}^{(2)}$ & I \\
\hline$P^{* \mu} u_{\mu} u^{\nu} P^{* \dagger}{ }_{\nu}$ & 5 & $-2 d_{3}^{(2)}$ & I & 7 & $-2 D_{3}^{(2)}$ & I \\
\hline$P^{* \mu} u^{\nu} u_{\mu} P^{* \dagger}{ }_{\nu}$ & 6 & $2 d_{3}^{(2)}$ & $-\tilde{c}_{5}^{(2)}$ & 8 & $2 D_{3}^{(2)}$ & $-\tilde{C}_{7}^{(2)}$ \\
\hline$P^{* \mu} u^{\nu} u_{\nu} P_{\mu}^{* \dagger}{ }_{\mu}$ & 7 & $2 d_{1}^{(2)}$ & $-\tilde{c}_{1}^{(2)}$ & 9 & $2 D_{1}^{(2)}$ & $-\tilde{C}_{1}^{(2)}$ \\
\hline$P^{* \mu} u^{\nu} u^{\lambda} D_{\nu \lambda} P^{* \dagger}{ }_{\mu}$ & 8 & $-2 d_{2}^{(2)}$ & $-\tilde{c}_{2}^{(2)}$ & 10 & $-2 D_{2}^{(2)}$ & $-\tilde{C}_{2}^{(2}$ \\
\hline$P^{* \mu}\left\langle u_{\mu} u^{\nu}\right\rangle P^{* \dagger}{ }_{\nu}$ & & & & 11 & 0 & 0 \\
\hline$P^{* \mu}\left\langle u^{\nu} u_{\nu}\right\rangle P_{\mu}^{* \dagger}$ & & & & 12 & $2 D_{4}^{(2)}$ & $-\tilde{C}_{3}^{(2)}$ \\
\hline$P^{* \mu}\left\langle u^{\nu} u^{\lambda}\right\rangle D_{\nu \lambda} P^{* \dagger}{ }_{\mu}$ & & & & 13 & $-2 D_{5}^{(2)}$ & $-\tilde{C}_{4}^{(2)}$ \\
\hline$i P^{* \mu} f_{+\mu}{ }^{\nu} P^{* \dagger}{ }_{\nu}$ & 9 & $4 d_{4}^{(2)}$ & I & 14 & $4 D_{6}^{(2)}$ & I \\
\hline$i P^{* \mu}\left\langle f_{+\mu}{ }^{\nu}\right\rangle P^{* \dagger}{ }_{\nu}$ & 10 & $4 d_{5}^{(2)}$ & I & & & \\
\hline$P^{* \mu} \chi_{+} P_{\mu}^{* \dagger}{ }_{\mu}$ & 11 & $2 d_{6}^{(2)}$ & $-\tilde{c}_{3}^{(2)}$ & 15 & $2 D_{7}^{(2)}$ & $-\tilde{C}_{5}^{(2)}$ \\
\hline$P^{* \mu}\left\langle\chi_{+}\right\rangle P^{* \dagger \dagger}{ }_{\mu}$ & 12 & $2 d_{7}^{(2)}$ & $-\tilde{c}_{4}^{(2)}$ & 16 & $2 D_{8}^{(2)}$ & $-\tilde{C}_{6}^{(2)}$ \\
\hline$\varepsilon^{\mu \nu \lambda \rho} P u_{\mu} u_{\nu} D_{\lambda} P^{* \dagger} \rho+$ H.c. & 13 & $-2 d_{3}^{(2)}$ & $\tilde{c}_{5}^{(2)}$ & 17 & $-2 D_{3}^{(2)}$ & $\tilde{C}_{7}^{(2)}$ \\
\hline$P f_{-}{ }^{\mu \nu} D_{\mu} P^{* \dagger}{ }_{\nu}+$ H.c. & 14 & 0 & 0 & 18 & $0^{3}$ & 0 \\
\hline$P h^{\mu \nu} D_{\mu} P_{\nu}^{* \dagger}+$ H.c. & 15 & 0 & 0 & 19 & 0 & 0 \\
\hline$i \varepsilon^{\mu \nu \lambda \rho} P f_{+\mu \nu} D_{\lambda} P_{\rho}^{* \dagger}+$ H.c. & 16 & $2 d_{4}^{(2)}$ & $\frac{1}{2} \tilde{c}_{9}^{(2)}$ & 20 & $2 D_{6}^{(2)}$ & $\frac{1}{2} \tilde{C}_{14}^{(2)}$ \\
\hline$i \varepsilon^{\mu \nu \lambda \rho} P\left\langle f_{+\mu \nu}\right\rangle D_{\lambda} P^{* \dagger} \rho+$ H.c. & 17 & $2 d_{5}^{(2)}$ & $\frac{1}{2} \tilde{c}_{10}^{(2)}$ & & & \\
\hline
\end{tabular}

The relations between $f_{Q}, g_{Q}$, and $g$ are found to be

$$
f_{Q}=2 g_{Q} M=-2 g M .
$$

TABLE IV. The $\mathcal{O}\left(p^{2}\right)$ order relativistic results. The columns 2,3 , and $4(5,6$, and 7$)$ are for the flavor $S U(2)$ [SU(3)] case. When a term $O_{n}$ is not given a label in the 2nd (5th) column, it is not independent and can be expressed with terms having a label in the 2 nd ( 5 th) column. "I" means that the structures of those terms are chosen as independent ones in the HQ limit. 
TABLE V. The $\mathcal{O}\left(p^{2}\right)$ order results in the HQ limit. When a term $P_{n}$ is not given a label in the 2 nd ( $3 \mathrm{rd}$ ) column, it is not independent and can be expressed with terms having a label in the 2nd (3rd) column.

\begin{tabular}{lcc}
\hline \hline$P_{n}$ & $S U(2)$ & $S U(3)$ \\
\hline$\left\langle H u^{\mu} u_{\mu} \bar{H}\right\rangle$ & 1 & 1 \\
$\left\langle H u^{\mu} u^{\nu} v_{\mu} v_{\nu} \bar{H}\right\rangle$ & 2 & 2 \\
$i\left\langle H u^{\mu} u^{\nu} \sigma_{\mu \nu} \bar{H}\right\rangle$ & 3 & 3 \\
$\left\langle H\left\langle u^{\mu} u_{\mu}\right\rangle \bar{H}\right\rangle$ & & 4 \\
$\left\langle H\left\langle u^{\mu} u^{\nu}\right\rangle v_{\mu} v_{\nu} \bar{H}\right\rangle$ & & 5 \\
$\left\langle H f_{+}{ }^{\mu \nu} \sigma_{\mu \nu} \bar{H}\right\rangle$ & 4 & 6 \\
$\left\langle H\left\langle f_{+}{ }^{\mu \nu}\right\rangle \sigma_{\mu \nu} \bar{H}\right\rangle$ & 5 & \\
$\left\langle H \chi_{+} \bar{H}\right\rangle$ & 6 & 7 \\
$\left\langle H\left\langle\chi_{+}\right\rangle \bar{H}\right\rangle$ & 7 & 8 \\
\hline \hline
\end{tabular}

The results at this order are applicable for both two- and three- flavor cases.

We show the $\mathcal{O}\left(p^{2}\right)$ Lagrangian in the relativistic form and HQ form in Tables IV and V, respectively. The 2nd and 5th columns of Table IV (2nd and 3rd columns of Table V) give the labels for each term in the flavor $S U(2)$ case and $S U(3)$ case, respectively. The 3rd and 6th columns of Table IV list the corresponding LECs in the HQ limit. The 4th and 7th columns of Table IV display the LEC relations between the relativistic terms according to the heavy quark symmetry, where "I" means that corresponding terms can be treated as the independent ones. Some monomials only happen in the either $S U(2)$ or $S U(3)$ case because of the Cayley-Hamilton relations and the convention of the trace of the vector source $v^{\mu}$. Hence, the other column is not labeled. Only a few analogous results in the references are found. The $\mathcal{O}\left(p^{2}\right)$ order $\mathcal{L}_{P P}$ is the same as that in Ref. [20].

\section{B. Results at the $\mathcal{O}\left(p^{3}\right)$ and $\mathcal{O}\left(p^{4}\right)$ orders}

The $\mathcal{O}\left(p^{3}\right)$ and $\mathcal{O}\left(p^{4}\right)$ order results are too long and we give them in Appendix A. The relativistic results are collected in Tables VII and IX while those in the HQ limit are listed in Tables VIII and X. The 3rd and 6th columns of Table VII show the corresponding LECs in the HQ limit while the 4th and 7th columns of the same table display the LEC relations between the relativistic terms obtained from the heavy quark symmetry. "I" in Tables VII and IX again means that the relevant terms are considered independent in the HQ limit. Some long expressions marked with "*" in Table VII are given explicitly below the table.

At present, we are not able to give the strict LEC relations for terms at the $\mathcal{O}\left(p^{4}\right)$ order. The $\mathcal{O}\left(p^{1}\right)$ order EOMs will appear because of the Schouten identity. Schouten identity can change the positions of some indexes and will give the factors as $D^{\mu} P^{* \dagger}{ }_{\mu}$ or $D^{2} \tilde{P}$. Hence the LECs at the $\mathcal{O}\left(p^{1}\right)$ order will appear in these relations. The exact relations need the determination of the inverse of a large symbolic matrix. Hence, we only mark the independent terms in the HQ limit in Table IX. In the table, the $52-57,241-253$, and 402-404 terms in the two-flavor case (97-99, 413-418, and 655 terms in the three-flavor case) are contact terms. In Table X, the 128-136 terms in the twoflavor case (209-212 terms in the three-flavor case) are contact terms.

\section{Discussions}

We have chosen the convention $\delta=1$ in presenting our final results. If one wants to use another convention, all the results need not be changed. For the $C$-parity transformation of $P^{*}$, we also use the "+" convention. Another convention only has an impact on $\mathcal{L}_{P P^{*}}$. Let us consider any $C$-, $P$ - and H.c.-invariant $\mathcal{L}_{P P^{*}}$ term

$$
\left(P O_{\mu} P^{* \dagger \mu}+\delta_{C} P^{* \mu} O_{C, \mu} P^{\dagger}\right)
$$

where $O_{\mu}$ is any possible structure, $O_{C, \mu}$ is an appropriate structure keeping the symmetry, and $\delta_{C}$ is the $C$-parity transformation factor of $P^{*}$. If one chooses an opposite sign of $\delta_{C}$, an extra $i$ factor is needed to keep Hermiticity.

\section{SUMMARY}

In the present paper, we extend our previous studies and construct the relativistic chiral Lagrangians for mesons with a heavy quark to one loop, both for the flavor $S U(3)$ case and for the flavor $S U(2)$ case. The chiral Lagrangians in the heavy quark limit are also obtained. The number of independent terms in the heavy quark limit is much less than that in the relativistic case, which is illustrated in Table VI. By comparing independent terms in the relativistic form and those in the HQ limit, one finds LEC relations at each order which result from the heavy quark symmetry. These relations would get corrections once the breaking of heavy quark symmetry is considered.

TABLE VI. Number of independent terms at each chiral order.

\begin{tabular}{|c|c|c|c|c|c|c|c|c|}
\hline \multirow[b]{2}{*}{ Chiral order } & \multicolumn{4}{|c|}{ Relativistic } & \multicolumn{4}{|c|}{ HQ limit } \\
\hline & $\mathcal{O}\left(p^{1}\right)$ & $\mathcal{O}\left(p^{2}\right)$ & $\mathcal{O}\left(p^{3}\right)$ & $\mathcal{O}\left(p^{4}\right)$ & $\mathcal{O}\left(p^{1}\right)$ & $\mathcal{O}\left(p^{2}\right)$ & $\mathcal{O}\left(p^{3}\right)$ & $\mathcal{O}\left(p^{4}\right)$ \\
\hline$S U(2)$ & 1 & 17 & 67 & 404 & 1 & 7 & 25 & 136 \\
\hline$S U(3)$ & 1 & 20 & 84 & 655 & 1 & 8 & 33 & 212 \\
\hline
\end{tabular}




\section{ACKNOWLEDGMENTS}

This work was supported by the National Science Foundation of China (NSFC) under Grants No. 11565004 and No. 11775132, Guangxi Science Foundation under Grants No. 2018GXNSFAA281180 and No. 2017AD22006, the special funding for Guangxi distinguished professors (Bagui Yingcai and Bagui Xuezhe) and High Level Innovation Team and Outstanding Scholar Program in Guangxi Colleges.

\section{APPENDIX: $\mathcal{O}\left(p^{3}\right)$ AND $\mathcal{O}\left(p^{4}\right)$ ORDER RESULTS}

The long relations in the fourth column of Table VII are

$$
\begin{aligned}
& \tilde{c}_{44}^{(3)}=\left(\tilde{c}_{10}^{(3)}+2 \tilde{c}_{11}^{(3)}-\tilde{c}_{26}^{(3)}\right)-2 \tilde{c}_{14}^{(3)}, \\
& \tilde{c}_{45}^{(3)}=-\left(\tilde{c}_{10}^{(3)}+2 \tilde{c}_{11}^{(3)}-\tilde{c}_{26}^{(3)}\right), \\
& \tilde{c}_{53}^{(3)}=\tilde{c}_{24}^{(3)}+\tilde{c}_{25}^{(3)}-\tilde{c}_{26}^{(3)} .
\end{aligned}
$$

TABLE VII. The $\mathcal{O}\left(p^{3}\right)$ order relativistic results. The columns 2,3 , and $4(5,6$, and 7$)$ are for the flavor $S U(2)$ [SU(3)] case. When a term $O_{n}$ is not given a label in the $2 \mathrm{nd}$ (5th) column, it is not independent and can be expressed with terms having a label in the $2 \mathrm{nd}(5 \mathrm{th})$

\begin{tabular}{|c|c|c|c|c|c|c|}
\hline$O_{n}$ & $S U(2)$ & $\tilde{c}_{n}^{(3)}$ & $\tilde{c}_{n}^{(3)}$ & $S U(3)$ & $\tilde{C}_{n}^{(3)}$ & $\tilde{C}_{n}^{(3)}$ \\
\hline$\varepsilon^{\mu \nu \lambda \rho} P u_{\mu} u_{\nu} u_{\lambda} D_{\rho} P^{\dagger}$ & 1 & $-2 d_{5}^{(3)}$ & I & 1 & $-2 D_{9}^{(3)}$ & I \\
\hline$\varepsilon^{\mu \nu \lambda \rho} P\left\langle u_{\mu} u_{\nu} u_{\lambda}\right\rangle D_{\rho} P^{\dagger}$ & & & & 2 & $-2 D_{10}^{(3)}$ & I \\
\hline$P u^{\mu} f_{-\mu}^{\nu} D_{\nu} P^{\dagger}+$ H.c. & 2 & $-2 d_{17}^{(3)}$ & I & 3 & $-2 D_{24}^{(3)}$ & I \\
\hline$P u^{\mu} h_{\mu}{ }^{\nu} D_{\nu} P^{\dagger}+$ H.c. & 3 & $-2 d_{20}^{(3)}$ & I & 4 & $-2 D_{27}^{(3)}$ & I \\
\hline$P u^{\mu} h^{\nu \lambda} D_{\mu \nu \lambda} P^{\dagger}+$ H.c. & 4 & $2 d_{21}^{(3)}$ & I & 5 & $2 D_{28}^{(3)}$ & I \\
\hline$i \varepsilon^{\mu \nu \lambda \rho} P f_{+\mu \nu} u_{\lambda} D_{\rho} P^{\dagger}+$ H.c. & 5 & $2 d_{12}^{(3)}$ & I & 6 & $2 D_{18}^{(3)}$ & I \\
\hline$i \varepsilon^{\mu \nu \lambda \rho} P\left\langle f_{+\mu \nu}\right\rangle u_{\lambda} D_{\rho} P^{\dagger}$ & 6 & $2 d_{13}^{(3)}$ & I & & & \\
\hline$i \varepsilon^{\mu \nu \lambda \rho} P\left\langle f_{+\mu \nu} u_{\lambda}\right\rangle D_{\rho} P^{\dagger}$ & & & & 7 & $2 D_{19}^{(3)}$ & I \\
\hline$i P \nabla^{\mu} f_{+\mu}{ }^{\nu} D_{\nu} P^{\dagger}$ & 7 & $2 d_{23}^{(3)}$ & I & 8 & $2 D_{33}^{(3)}$ & I \\
\hline$i P\left\langle\nabla^{\mu} f_{+\mu}{ }^{\nu}\right\rangle D_{\nu} P^{\dagger}$ & 8 & $2 d_{24}^{(3)}$ & I & & & \\
\hline$i P u^{\mu} \chi_{-} D_{\mu} P^{\dagger}+$ H.c. & 9 & $2 d_{16}^{(3)}$ & I & 9 & $2 D_{23}^{(3)}$ & I \\
\hline$\varepsilon^{\mu \nu \lambda \rho} P_{\mu}^{*} u_{\nu} u_{\lambda} u^{\sigma} D_{\rho} P^{* \dagger}{ }_{\sigma}+$ H.c. & 10 & $-2 d_{2}^{(3)}$ & I & 10 & $*$ & I \\
\hline$\varepsilon^{\mu \nu \lambda \rho} P_{\mu}^{*} u_{\nu} u_{\lambda} u^{\sigma} D_{\sigma} P^{* \dagger}{ }_{\rho}+$ H.c. & 11 & $d_{2}^{(3)}+d_{4}^{(3)}$ & I & 11 & $*$ & I \\
\hline$\varepsilon^{\mu \nu \lambda \rho} P_{\mu}^{*} u_{\nu} u^{\sigma} u_{\lambda} D_{\rho} P^{* \dagger}{ }_{\sigma}+$ H.c. & 12 & $-2 d_{2}^{(3)}-3 d_{5}^{(3)}$ & $\frac{3}{2} \tilde{c}_{1}^{(3)}+\tilde{c}_{10}^{(3)}$ & 12 & $-D_{9}^{(3)}$ & $\frac{1}{2} \tilde{C}_{1}^{(3)}$ \\
\hline$\varepsilon^{\mu \nu \lambda \rho} P^{*}{ }_{\mu} u_{\nu} u^{\sigma} u_{\sigma} D_{\lambda} P^{* \dagger}{ }_{\rho}+$ H.c. & 13 & $-2 d_{1}^{(3)}-d_{2}^{(3)}$ & I & 13 & $*$ & I \\
\hline$\varepsilon^{\mu \nu \lambda \rho} P^{*}{ }_{\mu} u^{\sigma} u_{\nu} u_{\lambda} D_{\rho} P^{* \dagger}{ }_{\sigma}+$ H.c. & & & & 14 & * & $-\tilde{C}_{1}^{(3)}-\tilde{C}_{10}^{(3)}$ \\
\hline$\varepsilon^{\mu \nu \lambda \rho} P^{*}{ }_{\mu} u_{\nu} u^{\sigma} u^{\delta} D_{\lambda \sigma \delta} P^{* \dagger}{ }_{\rho}+$ H.c. & 14 & $2 d_{3}^{(3)}+d_{4}^{(3)}$ & I & 15 & $*$ & I \\
\hline$\varepsilon^{\mu \nu \lambda \rho} P^{*}{ }_{\mu}\left\langle u_{\nu} u_{\lambda} u^{\sigma}\right\rangle D_{\rho} P^{* \dagger}{ }_{\sigma}+$ H.c. & & & & 16 & $3 D_{10}^{(3)}$ & $-\frac{3}{2} \tilde{C}_{2}^{(3)}$ \\
\hline$\varepsilon^{\mu \nu \lambda \rho} P^{*}{ }_{\mu}\left\langle u_{\nu} u^{\sigma}\right\rangle u_{\lambda} D_{\rho} P^{* \dagger}{ }_{\sigma}+$ H.c. & & & & 17 & $-2 D_{5}^{(3)}+D_{6}^{(3)}$ & $\mathrm{I}$ \\
\hline$\varepsilon^{\mu \nu \lambda \rho} P_{\mu}^{*}\left\langle u_{\nu} u^{\sigma}\right\rangle u_{\lambda} D_{\sigma} P^{* \dagger} \rho$ & & & & 18 & $*$ & I \\
\hline$\varepsilon^{\mu \nu \lambda \rho} P^{*}{ }_{\mu}\left\langle u_{\nu} u^{\sigma}\right\rangle u_{\sigma} D_{\lambda} P^{* \dagger} \rho$ & & & & 19 & $-2 D_{5}^{(3)}+3 D_{6}^{(3)}$ & I \\
\hline$\varepsilon^{\mu \nu \lambda \rho} P^{*}{ }_{\mu}\left\langle u_{\nu} u^{\sigma}\right\rangle u^{\delta} D_{\lambda \sigma \delta} P^{* \dagger} \rho$ & & & & 20 & $2 D_{7}^{(3)}-3 D_{8}^{(3)}$ & I \\
\hline$P^{* \mu} u_{\mu} f_{-}{ }^{\nu \lambda} D_{\nu} P^{* \dagger} \lambda+$ H.c. & 15 & $-2 d_{18}^{(3)}$ & I & 21 & $-2 D_{25}^{(3)}$ & I \\
\hline$P^{* \mu} u^{\nu} f_{-\mu}^{\lambda} D_{\nu} P^{* \dagger} \lambda+$ H.c. & 16 & $4 d_{19}^{(3)}$ & I & 22 & $4 D_{26}^{(3)}$ & I \\
\hline$P^{* \mu} u^{\nu} f_{-\mu}^{\lambda} D_{\lambda} P^{* \dagger}{ }_{\nu}+$ H.c. & 17 & $-2 d_{18}^{(3)}$ & $\tilde{c}_{15}^{(3)}$ & 23 & $-2 D_{25}^{(3)}$ & $\tilde{C}_{21}^{(3)}$ \\
\hline$P^{* \mu} u^{\nu} f_{-\nu}{ }^{\lambda} D_{\lambda} P^{* \dagger}{ }_{\mu}+$ H.c. & 18 & $2 d_{17}^{(3)}$ & $-\tilde{c}_{2}^{(3)}$ & 24 & $2 D_{24}^{(3)}$ & $-\tilde{C}_{3}^{(3)}$ \\
\hline$P^{* \mu} u_{\mu} h^{\nu \lambda} D_{\nu} P_{\lambda}^{* \dagger}+$ H.c. & 19 & $2 d_{22}^{(3)}$ & I & 25 & $2 D_{29}^{(3)}$ & I \\
\hline$P^{* \mu} u^{\nu} h_{\mu}{ }^{\lambda} D_{\nu} P^{* \dagger}{ }_{\lambda}+$ H.c. & 20 & 0 & 0 & 26 & 0 & 0 \\
\hline$P^{* \mu} u^{\nu} h_{\mu}{ }^{\lambda} D_{\lambda} P^{* \dagger}{ }_{\nu}+$ H.c. & 21 & $-2 d_{22}^{(3)}$ & $-\tilde{c}_{19}^{(3)}$ & 27 & $-2 D_{29}^{(3)}$ & $-\tilde{C}_{25}^{(3)}$ \\
\hline$P^{* \mu} u^{\nu} h_{\nu}{ }^{\lambda} D_{\lambda} P^{* \dagger}{ }_{\mu}+$ H.c. & 22 & $2 d_{20}^{(3)}$ & $-\tilde{c}_{3}^{(3)}$ & 28 & $2 D_{27}^{(3)}$ & $-\tilde{C}_{4}^{(3)}$ \\
\hline
\end{tabular}
column. "I" means that the structures of those terms are chosen as independent ones in the HQ limit. Relations marked with "**" are given below this table. 


\begin{tabular}{|c|c|c|c|c|c|c|}
\hline$O_{n}$ & $S U(2)$ & $\tilde{c}_{n}^{(3)}$ & $\tilde{c}_{n}^{(3)}$ & $S U(3)$ & $\tilde{C}_{n}^{(3)}$ & $\tilde{C}_{n}^{(3)}$ \\
\hline$P^{* \mu} u^{\nu} h^{\lambda \rho} D_{\nu \lambda \rho} P_{\mu}^{* \dagger}+$ H.c. & 23 & $-2 d_{21}^{(3)}$ & $-\tilde{c}_{4}^{(3)}$ & 29 & $-2 D_{28}^{(3)}$ & $-\tilde{C}_{5}^{(3)}$ \\
\hline$P^{* \mu}\left\langle u_{\mu} f_{-}^{\nu \lambda}\right\rangle D_{\nu} P_{\lambda}^{* \dagger}+$ H.c. & & & & 30 & $-2 D_{30}^{(3)}$ & I \\
\hline$P^{* \mu}\left\langle u^{\nu} f_{-\mu}^{\lambda}\right\rangle D_{\nu} P_{\lambda}^{* \dagger}$ & & & & 31 & $4 D_{31}^{(3)}$ & I \\
\hline$P^{* \mu}\left\langle u_{\mu} h^{\nu \lambda}\right\rangle D_{\nu} P_{\lambda}^{* \dagger}+$ H.c. & & & & 32 & $2 D_{32}^{(3)}$ & I \\
\hline$\varepsilon^{\mu \nu \lambda \rho} P_{\mu}^{*} \nabla_{\nu} f_{-\lambda}{ }^{\sigma} D_{\rho} P^{* \dagger}{ }_{\sigma}+$ H.c. & 24 & $2 d_{9}^{(3)}$ & I & 33 & $2 D_{15}^{(3)}$ & I \\
\hline$\varepsilon^{\mu \nu \lambda \rho} P_{\mu}^{*} \nabla_{\nu} f_{-\lambda}{ }^{\sigma} D_{\sigma} P_{\rho}^{* \dagger} \rho$ & 25 & $-2 d_{9}^{(3)}+2 d_{10}^{(3)}$ & I & 34 & $-2 D_{15}^{(3)}+2 D_{16}^{(3)}$ & I \\
\hline$\varepsilon^{\mu \nu \lambda \rho} P_{\mu}^{*} \nabla_{\nu} h^{\sigma \delta} D_{\lambda \sigma \delta} P_{\rho}^{* \dagger}$ & 26 & $2 d_{11}^{(3)}$ & I & 35 & $2 D_{17}^{(3)}$ & I \\
\hline$i \varepsilon^{\mu \nu \lambda \rho} P_{\mu}^{*} f_{+\nu \lambda} u^{\sigma} D_{\rho} P_{\sigma}^{* \dagger}+$ H.c. & 27 & $-2 d_{12}^{(3)}$ & $-\tilde{c}_{5}^{(3)}$ & 36 & $-2 D_{18}^{(3)}$ & $-\tilde{C}_{6}^{(3)}$ \\
\hline$i \varepsilon^{\mu \nu \lambda \rho} P_{\mu}^{*} f_{+\nu \lambda} u^{\sigma} D_{\sigma} P_{\rho}^{* \dagger}+$ H.c. & 28 & $-d_{7}^{(3)}$ & I & 37 & $-D_{12}^{(3)}$ & $\mathrm{I}^{\mathrm{O}}$ \\
\hline$i \varepsilon^{\mu \nu \lambda \rho} P^{*}{ }_{\mu} f_{+\nu}{ }^{\sigma} u_{\lambda} D_{\rho} P^{* \dagger}{ }_{\sigma}+$ H.c. & 29 & $4 d_{12}^{(3)}$ & $2 \tilde{c}_{5}^{(3)}$ & 38 & $4 D_{18}^{(3)}$ & $2 \tilde{C}_{6}^{(3)}$ \\
\hline$i \varepsilon^{\mu \nu \lambda \rho} P_{\mu}^{*} f_{+\nu}{ }^{\sigma} u_{\sigma} D_{\lambda} P_{\rho}^{* \dagger}+$ H.c. & 30 & $2 d_{6}^{(3)}$ & I & 39 & $2 D_{11}^{(3)}$ & I \\
\hline$i \varepsilon^{\mu \nu \lambda \rho} P_{\mu}^{*}\left\langle f_{+\nu \lambda}\right\rangle u^{\sigma} D_{\rho} P^{* \dagger}{ }_{\sigma}+$ H.c. & 31 & $-d_{13}^{(3)}$ & $-\frac{1}{2} \tilde{c}_{6}^{(3)}$ & & & \\
\hline$i \varepsilon^{\mu \nu \lambda \rho} P_{\mu}^{*}\left\langle f_{+\nu}{ }^{\sigma}\right\rangle u_{\lambda} D_{\rho} P^{* \dagger}{ }_{\sigma}+$ H.c. & 32 & $2 d_{13}^{(3)}$ & $\tilde{c}_{6}^{(3)}$ & & & \\
\hline$i \varepsilon^{\mu \nu \lambda \rho} P_{\mu}^{*}\left\langle f_{+\nu \lambda} u^{\sigma}\right\rangle D_{\rho} P^{* \dagger}{ }_{\sigma}+$ H.c. & & & & 40 & $-D_{19}^{(3)}$ & $-\frac{1}{2} \tilde{C}_{7}^{(3)}$ \\
\hline$i \varepsilon^{\mu \nu \lambda \rho} P_{\mu}^{*}\left\langle f_{+\nu}{ }^{\sigma} u_{\lambda}\right\rangle D_{\rho} P^{* \dagger}{ }_{\sigma}+$ H.c. & & & & 41 & $2 D_{19}^{(3)}$ & $\tilde{C}_{7}^{(3)}$ \\
\hline$i P^{* \mu} \nabla_{\mu} f_{+}{ }^{\nu \lambda} D_{\nu} P_{\lambda}^{* \dagger}+$ H.c. & 33 & 0 & 0 & 42 & 0 & 0 \\
\hline$i P^{* \mu} \nabla^{\nu} f_{+\nu}^{\lambda} D_{\lambda} P^{* \dagger}{ }_{\mu}$ & 34 & $-2 d_{23}^{(3)}$ & $-\tilde{c}_{7}^{(3)}$ & 43 & $-2 D_{33}^{(3)}$ & $-\tilde{C}_{8}^{(3)}$ \\
\hline$i P^{* \mu}\left\langle\nabla_{\mu} f_{+}{ }^{\nu \lambda}\right\rangle D_{\nu} P^{* \dagger}{ }_{\lambda}+$ H.c. & 35 & 0 & 0 & & & \\
\hline$i P^{* \mu}\left\langle\nabla^{\nu} f_{+\nu}{ }^{\lambda}\right\rangle D_{\lambda} P_{\mu}^{* \dagger}$ & 36 & $-2 d_{24}^{(3)}$ & $-\tilde{c}_{8}^{(3)}$ & & & \\
\hline$\varepsilon^{\mu \nu \lambda \rho} P_{\mu}^{*} u_{\nu} \chi_{+} D_{\lambda} P^{* \dagger}{ }_{\rho}+$ H.c. & 37 & $-2 d_{14}^{(3)}$ & I & 44 & $-2 D_{20}^{(3)}$ & I \\
\hline$\varepsilon^{\mu \nu \lambda \rho} P_{\mu}^{*}\left\langle u_{\nu} \chi_{+}\right\rangle D_{\lambda} P_{\rho}^{* \dagger} \rho$ & 38 & $-2 d_{15}^{(3)}$ & I & 45 & $-2 D_{21}^{(3)}$ & I \\
\hline$\varepsilon^{\mu \nu \lambda \rho} P_{\mu}^{*}\left\langle\chi_{+}\right\rangle u_{\nu} D_{\lambda} P^{* \dagger} \rho$ & & & & 46 & $-2 D_{22}^{(3)}$ & I \\
\hline$i P^{* \mu} u^{\nu} \chi_{-} D_{\nu} P_{\mu}^{* \dagger}+$ H.c. & 39 & $-2 d_{16}^{(3)}$ & $-\tilde{c}_{9}^{(3)}$ & 47 & $-2 D_{23}^{(3)}$ & $-\tilde{C}_{9}^{(3)}$ \\
\hline$i \varepsilon^{\mu \nu \lambda \rho} P_{\mu}^{*} \nabla_{\nu} \chi_{-} D_{\lambda} P_{\rho}^{* \dagger}$ & 40 & $-2 d_{25}^{(3)}$ & I & 48 & $-2 D_{13}^{(3)}$ & I \\
\hline$i \varepsilon^{\mu \nu \lambda \rho} P_{\mu}^{*}\left\langle\nabla_{\nu} \chi_{-}\right\rangle D_{\lambda} P_{\rho}^{* \dagger} \rho$ & 41 & $-2 d_{8}^{(3)}$ & I & 49 & $-2 D_{14}^{(3)}$ & I \\
\hline$P u^{\mu} u_{\mu} u_{\nu}^{\nu} P_{\nu}^{* \dagger}+$ H.c. & 42 & $4 d_{1}^{(3)}$ & $\tilde{c}_{10}^{(3)}-2 \tilde{c}_{13}^{(3)}$ & 50 & $2 D_{1}^{(3)}$ & $*$ \\
\hline$P u^{\mu} u^{\nu} u_{\mu} P_{\nu}^{* \dagger}+$ H.c. & 43 & $2 d_{2}^{(3)}$ & $-\tilde{c}_{10}^{(3)}$ & 51 & $2 D_{2}^{(3)}$ & * \\
\hline$P u^{\mu} u^{\nu} u_{\nu} P_{\mu}^{* \dagger}+$ H.c. & & & & 52 & $2 D_{1}^{(3)}$ & $*$ \\
\hline$P u^{\mu} u^{\nu} u^{\lambda} D_{\mu \nu} P_{\lambda}^{* \dagger}+$ H.c. & 44 & $-4 d_{3}^{(3)}-2 d_{11}^{(3)}$ & $*$ & 53 & $*$ & $*$ \\
\hline$P u^{\mu} u^{\nu} u^{\lambda} D_{\mu \lambda} P_{\nu}^{* \dagger}+$ H.c. & 45 & $-2 d_{4}^{(3)}+2 d_{11}^{(3)}$ & $*$ & 54 & $*$ & $*$ \\
\hline$P u^{\mu} u^{\nu} u^{\lambda} D_{\nu \lambda} P^{* \dagger}{ }_{\mu}+$ H.c. & & & & 55 & $*$ & $*$ \\
\hline$P\left\langle u^{\mu} u_{\mu}\right\rangle u_{\nu}^{\nu} P_{\nu}^{* \dagger}+$ H.c. & & & & 56 & $2 D_{5}^{(3)}$ & $-\frac{3}{2} \tilde{C}_{17}^{(3)}+\frac{1}{2} \tilde{C}_{19}^{(3)}$ \\
\hline$P\left\langle u^{\mu} u_{\mu} u^{\nu}\right\rangle P_{\nu}^{* \dagger}+$ H.c. & & & & 57 & $2 D_{6}^{(3)}$ & $-\tilde{C}_{17}^{(3)}+\tilde{C}_{19}^{(3)}$ \\
\hline$P\left\langle u^{\mu} u^{\nu}\right\rangle u^{\lambda} D_{\mu \nu} P_{\lambda}^{* \dagger}+$ H.c. & & & & 58 & $D_{7}^{(3)}$ & $*$ \\
\hline$P\left\langle u^{\mu} u^{\nu} u^{\lambda}\right\rangle D_{\mu \nu} P_{\lambda}^{* \dagger}+$ H.c. & & & & 59 & $2 D_{7}^{(3)}-2 D_{8}^{(3)}$ & $*$ \\
\hline$\varepsilon^{\mu \nu \lambda \rho} P u_{\mu} f_{-\nu \lambda} P_{\rho}^{* \dagger}+$ H.c. & 46 & $-2 d_{19}^{(3)}$ & $-\frac{1}{2} \tilde{c}_{16}^{(3)}$ & 60 & $-2 D_{26}^{(3)}$ & $-\frac{1}{2} \tilde{C}_{22}^{(3)}$ \\
\hline$\varepsilon^{\mu \nu \lambda \rho} P f_{-\mu \nu} u_{\lambda} P_{\rho}^{* \dagger}+$ H.c. & 47 & $-d_{18}^{(3)}$ & $\frac{1}{2} \tilde{c}_{15}^{(3)}$ & 61 & $-D_{25}^{(3)}$ & $\frac{1}{2} \tilde{C}_{21}^{(3)}$ \\
\hline$\varepsilon^{\mu \nu \lambda \rho} P u_{\mu} f_{-\nu}^{\sigma} D_{\lambda \sigma} P_{\rho}^{* \dagger}+$ H.c. & 48 & $2 d_{18}^{(3)}-4 d_{19}^{(3)}$ & $-\tilde{c}_{15}^{(3)}-\tilde{c}_{16}^{(3)}$ & 62 & $2 D_{25}^{(3)}-4 D_{26}^{(3)}$ & $-\tilde{C}_{21}^{(3)}-\tilde{C}_{22}^{(3)}$ \\
\hline$\varepsilon^{\mu \nu \lambda \rho} P f_{-\mu \nu} u^{\sigma} D_{\lambda \sigma} P_{\rho}^{* \dagger}+$ H.c. & 49 & $-d_{18}^{(3)}+2 d_{19}^{(3)}$ & $\frac{1}{2} \tilde{c}_{15}^{(3)}+\frac{1}{2} \tilde{c}_{16}^{(3)}$ & 63 & $-D_{25}^{(3)}+2 D_{26}^{(3)}$ & $\frac{1}{2} \tilde{C}_{21}^{(3)}+\frac{1}{2} \tilde{C}_{22}^{(3)}$ \\
\hline$\varepsilon^{\mu \nu \lambda \rho} P u_{\mu} h_{\nu}{ }^{\sigma} D_{\lambda \sigma} P_{\rho}^{* \dagger}+$ H.c. & 50 & $2 d_{22}^{(3)}$ & $\tilde{c}_{19}^{(3)}$ & 64 & $2 D_{29}^{(3)}$ & $\tilde{C}_{25}^{(3)}$ \\
\hline$\varepsilon^{\mu \nu \lambda \rho} P h_{\mu}{ }^{\sigma} u_{\nu} D_{\lambda \sigma} P_{\rho}^{* \dagger}+$ H.c. & 51 & $-2 d_{22}^{(3)}$ & $-\tilde{c}_{19}^{(3)}$ & 65 & $-2 D_{29}^{(3)}$ & $-\tilde{C}_{25}^{(3)}$ \\
\hline$\varepsilon^{\mu \nu \lambda \rho} P\left\langle u_{\mu} f_{-\nu \lambda}\right\rangle P_{\rho}^{* \dagger}+$ H.c. & & & & 66 & $-2 D_{31}^{(3)}$ & $-\frac{1}{2} \tilde{C}_{31}^{(3)}$ \\
\hline$\varepsilon^{\mu \nu \lambda \rho} P\left\langle u_{\mu} f_{-\nu}^{\sigma}\right\rangle D_{\lambda \sigma} P_{\rho}^{* \dagger}+$ H.c. & & & & 67 & $2 D_{30}^{(3)}-4 D_{31}^{(3)}$ & $-\tilde{C}_{30}^{(3)}-\tilde{C}_{31}^{(3)}$ \\
\hline
\end{tabular}


TABLE VII. (Continued)

\begin{tabular}{|c|c|c|c|c|c|c|}
\hline$O_{n}$ & $S U(2)$ & $\tilde{c}_{n}^{(3)}$ & $\tilde{c}_{n}^{(3)}$ & $S U(3)$ & $\tilde{C}_{n}^{(3)}$ & $\tilde{C}_{n}^{(3)}$ \\
\hline$\varepsilon^{\mu \nu \lambda \rho} P\left\langle u_{\mu} h_{\nu}{ }^{\sigma}\right\rangle D_{\lambda \sigma} P_{\rho}^{* \dagger}+$ H.c. & & & & 68 & $2 D_{32}^{(3)}$ & $\tilde{C}_{32}^{(3)}$ \\
\hline$P \nabla^{\mu} f_{-\mu}^{\nu} P_{\nu}^{* \dagger}+$ H.c. & 52 & $2 d_{9}^{(3)}$ & $\tilde{c}_{24}^{(3)}$ & 69 & $2 D_{15}^{(3)}$ & $\tilde{C}_{33}^{(3)}$ \\
\hline$P \nabla^{\mu} f_{-}{ }^{\nu \lambda} D_{\mu \nu} P_{\lambda}^{* \dagger}+$ H.c. & 53 & $2 d_{10}^{(3)}-2 d_{11}^{(3)}$ & $*$ & 70 & $2 D_{16}^{(3)}-2 D_{17}^{(3)}$ & $*$ \\
\hline$P \nabla^{\mu} h^{\nu \lambda} D_{\mu \nu} P_{\lambda}^{* \dagger}+$ H.c. & 54 & $-2 d_{11}^{(3)}$ & $-\tilde{c}_{26}^{(3)}$ & 71 & $-2 D_{17}^{(3)}$ & $-\tilde{C}_{35}^{(3)}$ \\
\hline$i P f_{+}{ }^{\mu \nu} u_{\mu} P^{* \dagger}{ }_{\nu}+$ H.c. & 55 & $2 d_{6}^{(3)}$ & $\tilde{c}_{30}^{(3)}$ & 72 & $2 D_{11}^{(3)}$ & $\tilde{C}_{39}^{(3)}$ \\
\hline$i P u^{\mu} f_{+\mu}{ }^{\nu} P^{* \dagger}{ }_{\nu}+$ H.c. & 56 & $-2 d_{6}^{(3)}$ & $-\tilde{c}_{30}^{(3)}$ & 73 & $-2 D_{11}^{(3)}$ & $-\tilde{C}_{39}^{(3)}$ \\
\hline$i P f_{+}{ }^{\mu \nu} u^{\lambda} D_{\mu \lambda} P^{* \dagger}{ }_{\nu}+$ H.c. & 57 & $2 d_{7}^{(3)}-2 d_{11}^{(3)}$ & $-\tilde{c}_{26}^{(3)}-2 \tilde{c}_{28}^{(3)}$ & 74 & $2 D_{12}^{(3)}-2 D_{17}^{(3)}$ & $-\tilde{C}_{35}^{(3)}-2 \tilde{C}_{37}^{(3)}$ \\
\hline$i P u^{\mu} f_{+}{ }^{\nu \lambda} D_{\mu \nu} P_{\lambda}^{* \dagger}+$ H.c. & 58 & $-2 d_{7}^{(3)}+2 d_{11}^{(3)}$ & $\tilde{c}_{26}^{(3)}+2 \tilde{c}_{28}^{(3)}$ & 75 & $-2 D_{12}^{(3)}+2 D_{17}^{(3)}$ & $\tilde{C}_{35}^{(3)}+2 \tilde{C}_{37}^{(3)}$ \\
\hline$i P\left\langle f_{+}{ }^{\mu \nu}\right\rangle u_{\mu} P^{* \dagger}{ }_{\nu}+$ H.c. & 59 & 0 & 0 & & & \\
\hline$i P\left\langle f_{+}{ }^{\mu \nu}\right\rangle u^{\lambda} D_{\mu \lambda} P_{\nu}^{* \dagger}{ }_{\nu}+$ H.c. & 60 & 0 & 0 & & & \\
\hline$i P\left\langle f_{+}{ }^{\mu \nu} u_{\mu}\right\rangle P^{* \dagger}{ }_{\nu}+$ H.c. & & & & 76 & 0 & 0 \\
\hline$i P\left\langle f_{+}{ }^{\mu \nu} u^{\lambda}\right\rangle D_{\mu \lambda} P^{* \dagger}{ }_{\nu}+$ H.c. & & & & 77 & 0 & 0 \\
\hline$i \varepsilon^{\mu \nu \lambda \rho} P \nabla_{\mu} f_{+\nu}{ }^{\sigma} D_{\lambda \sigma} P^{* \dagger} \rho+$ H.c. & 61 & 0 & 0 & 78 & 0 & 0 \\
\hline$i \varepsilon^{\mu \nu \lambda \rho} P\left\langle\nabla_{\mu} f_{+\nu}{ }^{\sigma}\right\rangle D_{\lambda \sigma} P^{* \dagger}{ }_{\rho}+$ H.c. & 62 & 0 & 0 & & & \\
\hline$P u^{\mu} \chi_{+} P_{\mu}^{* \dagger}+$ H.c. & 63 & $2 d_{14}^{(3)}$ & $-\tilde{c}_{37}^{(3)}$ & 79 & $2 D_{20}^{(3)}$ & $-\tilde{C}_{44}^{(3)}$ \\
\hline$P \chi_{+} u^{\mu} P_{\mu}^{* \dagger}+$ H.c. & 64 & $2 d_{14}^{(3)}$ & $-\tilde{c}_{37}^{(3)}$ & 80 & $2 D_{20}^{(3)}$ & $-\tilde{C}_{44}^{(3)}$ \\
\hline$P\left\langle u^{\mu} \chi_{+}\right\rangle P_{\mu}^{* \dagger}+$ H.c. & 65 & $2 d_{15}^{(3)}$ & $-\tilde{c}_{38}^{(3)}$ & 81 & $2 D_{21}^{(3)}$ & $-\tilde{C}_{45}^{(3)}$ \\
\hline$P\left\langle\chi_{+}\right\rangle u^{\mu} P_{\mu}^{* \dagger}+$ H.c. & & & & 82 & $2 D_{22}^{(3)}$ & $-\tilde{C}_{46}^{(3)}$ \\
\hline$i P \nabla^{\mu} \chi_{-} P_{\mu}^{* \dagger}+$ H.c. & 66 & $2 d_{25}^{(3)}$ & $-\tilde{c}_{40}^{(3)}$ & 83 & $2 D_{13}^{(3)}$ & $-\tilde{C}_{48}^{(3)}$ \\
\hline$i P\left\langle\nabla^{\mu} \chi_{-}\right\rangle P_{\mu}^{* \dagger}+$ H.c. & 67 & $2 d_{8}^{(3)}$ & $-\tilde{c}_{41}^{(3)}$ & 84 & $2 D_{14}^{(3)}$ & $-\tilde{C}_{49}^{(3)}$ \\
\hline
\end{tabular}

TABLE VIII. The $\mathcal{O}\left(p^{3}\right)$ order results in the HQ limit. When a term $P_{n}$ is not given a label in the 2 nd or 5 th (3rd or 6th) column, it is not independent and can be expressed with terms having a label in the 2nd and 5th (3rd and 6th) columns.

\begin{tabular}{|c|c|c|c|c|c|}
\hline$P_{n}$ & $S U(2)$ & $S U(3)$ & $P_{n}$ & $S U(2)$ & $S U(3)$ \\
\hline$\left\langle\left\langle u^{\mu} u_{\mu} u^{\nu} \gamma_{5} \gamma_{\nu} \bar{H}\right\rangle+\right.$ H.c. & 1 & 1 & $\varepsilon^{\mu \nu \lambda \rho}\left\langle H\left\langle f_{+\mu \nu}\right\rangle u_{\lambda} v_{\rho} \bar{H}\right\rangle$ & 13 & \\
\hline$\left\langle H u^{\mu} u^{\nu} u_{\mu} \gamma_{5} \gamma_{\nu} \bar{H}\right\rangle$ & 2 & 2 & $\varepsilon^{\mu \nu \lambda \rho}\left\langle H\left\langle f_{+\mu \nu} u_{\lambda}\right\rangle v_{\rho} \bar{H}\right\rangle$ & & 19 \\
\hline$\left\langle H u^{\mu} u^{\nu} u^{\lambda} \gamma_{5} \gamma_{\mu} v_{\nu} v_{\lambda} \bar{H}\right\rangle+$ H.c. & 3 & 3 & $\left\langle H u^{\mu} \chi_{+} \gamma_{5} \gamma_{\mu} \bar{H}\right\rangle+$ H.c. & 14 & 20 \\
\hline$\left\langle H u^{\mu} u^{\nu} u^{\lambda} \gamma_{5} \gamma_{\nu} v_{\mu} v_{\lambda} \bar{H}\right\rangle$ & 4 & 4 & $\left\langle H\left\langle u^{\mu} \chi_{+}\right\rangle \gamma_{5} \gamma_{\mu} \bar{H}\right\rangle$ & 15 & 21 \\
\hline$\left\langle H\left\langle u^{\mu} u_{\mu}\right\rangle u^{\nu} \gamma_{5} \gamma_{\nu} \bar{H}\right\rangle$ & & 5 & $\left\langle H\left\langle\chi_{+}\right\rangle u^{\mu} \gamma_{5} \gamma_{\mu} \bar{H}\right\rangle$ & & 22 \\
\hline$\left\langle H\left\langle u^{\mu} u_{\mu} u^{\nu}\right\rangle \gamma_{5} \gamma_{\nu} \bar{H}\right\rangle$ & & 6 & $\left\langle H u^{\mu} \chi_{-} v_{\mu} \bar{H}\right\rangle+$ H.c. & 16 & 23 \\
\hline$\left\langle H\left\langle u^{\mu} u^{\nu}\right\rangle u^{\lambda} \gamma_{5} \gamma_{\mu} v_{\nu} v_{\lambda} \bar{H}\right\rangle$ & & 7 & $i\left\langle H u^{\mu} f_{-\mu}{ }^{\nu} v_{\nu} \bar{H}\right\rangle+$ H.c. & 17 & 24 \\
\hline$\left\langle H\left\langle u^{\mu} u^{\nu} u^{\lambda}\right\rangle \gamma_{5} \gamma_{\mu} v_{\nu} v_{\lambda} \bar{H}\right\rangle$ & & 8 & $\left\langle H u^{\mu} f_{-}{ }^{\nu \lambda} \sigma_{\mu \nu} v_{\lambda} \bar{H}\right\rangle+$ H.c. & 18 & 25 \\
\hline$i \varepsilon^{\mu \nu \lambda \rho}\left\langle H u_{\mu} u_{\nu} u_{\lambda} v_{\rho} \bar{H}\right\rangle$ & 5 & 9 & $\left\langle H u^{\mu} f_{-}{ }^{\nu \lambda} \sigma_{\nu \lambda} v_{\mu} \bar{H}\right\rangle+$ H.c. & 19 & 26 \\
\hline$i \varepsilon^{\mu \nu \lambda \rho}\left\langle H\left\langle u_{\mu} u_{\nu} u_{\lambda}\right\rangle v_{\rho} \bar{H}\right\rangle$ & & 10 & $i\left\langle H u^{\mu} h_{\mu}{ }^{\nu} v_{\nu} \bar{H}\right\rangle+$ H.c. & 20 & 27 \\
\hline$i\left\langle H f_{+}{ }^{\mu \nu} u_{\mu} \gamma_{5} \gamma_{\nu} \bar{H}\right\rangle+$ H.c. & 6 & 11 & $i\left\langle H u^{\mu} h^{\nu \lambda} v_{\mu} v_{\nu} v_{\lambda} \bar{H}\right\rangle+$ H.c. & 21 & 28 \\
\hline$i\left\langle H f_{+}{ }^{\mu \nu} u^{\lambda} \gamma_{5} \gamma_{\mu} v_{\nu} v_{\lambda} \bar{H}\right\rangle+$ H.c. & 7 & 12 & $\left\langle H u^{\mu} h^{\nu \lambda} \sigma_{\mu \nu} v_{\lambda} \bar{H}\right\rangle+$ H.c. & 22 & 29 \\
\hline$i\left\langle H \nabla^{\mu} \chi_{-} \gamma_{5} \gamma_{\mu} \bar{H}\right\rangle$ & & 13 & $\left\langle H\left\langle u^{\mu} f_{-}{ }^{\nu \lambda}\right\rangle \sigma_{\mu \nu} v_{\lambda} \bar{H}\right\rangle$ & & 30 \\
\hline$i\left\langle H\left\langle\nabla^{\mu} \chi_{-}\right\rangle \gamma_{5} \gamma_{\mu} \bar{H}\right\rangle$ & 8 & 14 & $\left\langle H\left\langle u^{\mu} f_{-}{ }^{\nu \lambda}\right\rangle \sigma_{\nu \lambda} v_{\mu} \bar{H}\right\rangle$ & & 31 \\
\hline$\left\langle H \nabla^{\mu} f_{-\mu}{ }^{\nu} \gamma_{5} \gamma_{\nu} \bar{H}\right\rangle$ & 9 & 15 & $\left\langle H\left\langle u^{\mu} h^{\nu \lambda}\right\rangle \sigma_{\mu \nu} v_{\lambda} \bar{H}\right\rangle$ & & 32 \\
\hline$\left\langle H \nabla^{\mu} f_{-}{ }^{\nu \lambda} \gamma_{5} \gamma_{\nu} v_{\mu} v_{\lambda} \bar{H}\right\rangle$ & 10 & 16 & $\left\langle H \nabla^{\mu} f_{+\mu}{ }^{\nu} v_{\nu} \bar{H}\right\rangle$ & 23 & 33 \\
\hline$\left\langle H \nabla^{\mu} h^{\nu \lambda} \gamma_{5} \gamma_{\mu} v_{\nu} v_{\lambda} \bar{H}\right\rangle$ & 11 & 17 & $\left\langle H\left\langle\nabla^{\mu} f_{+\mu}^{\mu}{ }^{\nu}\right\rangle v_{\nu} \bar{H}\right\rangle$ & 24 & \\
\hline$\varepsilon^{\mu \nu \lambda \rho}\left\langle H f_{+\mu \nu} u_{\lambda} v_{\rho} \bar{H}\right\rangle+$ H.c. & 12 & 18 & $i\left\langle H \nabla^{\mu} \chi_{-} \gamma_{5} \gamma_{\mu} \bar{H}\right\rangle$ & 25 & \\
\hline
\end{tabular}


TABLE IX. The $\mathcal{O}\left(p^{4}\right)$ order relativistic results. The columns $2,3,7$, and $8(4,5,9$, and 10) are for the flavor $S U(2)$ [SU(3)] case. When a term $O_{n}$ is not given a label in the 2nd or 7th (4th or 8th) column, it is not independent and can be expressed with terms having a label in the $S U(2)(S U(3))$ case. "I" means that the structures of those terms are chosen as independent ones in the HQ limit. "P." stands for parity-transformed part.

\begin{tabular}{|c|c|c|c|c|c|c|c|c|c|}
\hline$O_{n}$ & $S U(2)$ & $\tilde{c}_{n}^{(4)}$ & $S U(3)$ & $\tilde{C}_{n}^{(4)}$ & $O_{n}$ & $S U(2)$ & $\tilde{c}_{n}^{(4)}$ & $S U(3)$ & $\tilde{C}_{n}^{(4)}$ \\
\hline$P\left\langle u^{\mu} u_{\mu}\right\rangle u^{\nu} u_{\nu} P^{\dagger}$ & 1 & I & 1 & I & $i P^{* \mu} \nabla_{\mu} \nabla^{\nu} f_{+}^{\lambda \rho} D_{\nu \lambda} P^{* \dagger}{ }_{\rho}+$ H.c. & 182 & I & 334 & I \\
\hline$P\left\langle u^{\mu} u^{\nu}\right\rangle u_{\mu} u_{\nu} P^{\dagger}$ & 2 & I & 2 & $\mathrm{I}$ & $i P^{* \mu} \nabla^{\mu} \nabla^{\lambda} f_{+\mu}{ }^{\rho} D_{\nu \lambda} P^{* \dagger} \rho$ & 183 & I & 335 & I \\
\hline$P\left\langle u^{\mu} u_{\mu}\right\rangle u^{\nu} u^{\lambda} D_{\nu \lambda} P^{\dagger}$ & 3 & I & 3 & I & $P^{* \mu} f_{+\mu}^{\nu} f_{+\nu}^{\lambda} P^{* \dagger} \lambda$ & 184 & I & 336 & I \\
\hline$P\left\langle u^{\mu} u^{\nu}\right\rangle u_{\mu} u^{\lambda} D_{\nu \lambda} P^{\dagger}+$ H.c. & 4 & I & 4 & I & $P^{* \mu} f_{+}{ }^{\nu \lambda} f_{+\mu \nu} P^{* \dagger}{ }_{\lambda}$ & 185 & & 337 & \\
\hline$P\left\langle u^{\mu} u^{\nu}\right\rangle u^{\lambda} u_{\lambda} D_{\mu \nu} P^{\dagger}$ & & & 5 & I & $P^{* \mu} f_{+}{ }^{\nu \lambda} f_{+\nu \lambda} P^{* \dagger}{ }_{\mu}$ & 186 & & 338 & \\
\hline$P\left\langle u^{\mu} u^{\nu}\right\rangle u^{\lambda} u^{\rho} D_{\mu \nu \lambda \rho} P^{\dagger}$ & 5 & I & 6 & I & $P^{* \mu} f_{+\mu}{ }^{\nu} f_{+}^{\lambda \rho} D_{\nu \lambda} P^{* \dagger} \rho$ & 187 & I & 339 & I \\
\hline$P\left\langle u^{\mu} u_{\mu} u^{\nu}\right\rangle u_{\nu} P^{\dagger}$ & & & 7 & I & $P^{* \mu} f_{+}{ }^{\nu \lambda} f_{+\mu}{ }^{\rho} D_{\nu \rho} P^{* \dagger}{ }_{\lambda}$ & 188 & & 340 & \\
\hline$P\left\langle u^{\mu} u_{\mu} u^{\nu}\right\rangle u^{\lambda} D_{\nu \lambda} P^{\dagger}$ & & & 8 & I & $P^{* \mu} f_{+}{ }^{\nu \lambda} f_{+\nu}^{\rho} D_{\lambda \rho} P^{* \dagger}{ }_{\mu}$ & 189 & & 341 & \\
\hline$P\left\langle u^{\mu} u^{\nu} u^{\lambda}\right\rangle u_{\mu} D_{\nu \lambda} P^{\dagger}$ & & & 9 & I & $P^{* \mu}\left\langle f_{+\mu}{ }^{\nu}\right\rangle f_{+\nu}{ }^{\lambda} P^{* \dagger} \lambda+$ H.c. & 190 & & & \\
\hline$P\left\langle u^{\mu} u^{\nu} u^{\lambda}\right\rangle u^{\rho} D_{\mu \nu \lambda \rho} P^{\dagger}$ & & & 10 & I & $P^{* \mu}\left\langle f_{+}{ }^{\nu \lambda}\right\rangle f_{+\nu \lambda} P^{* \dagger}{ }_{\mu}$ & 191 & & & \\
\hline$P\left\langle u^{\mu} u_{\mu} u^{\nu} u_{\nu}\right\rangle P^{\dagger}$ & & & 11 & I & $P^{* \mu}\left\langle f_{+\mu}{ }^{\nu}\right\rangle f_{+}^{\lambda \rho} D_{\nu \lambda}^{\mu} P^{* \dagger}{ }_{\rho}+$ H.c. & 192 & & & \\
\hline$P\left\langle u^{\mu} u^{\nu} u_{\mu} u_{\nu}\right\rangle P^{\dagger}$ & & & 12 & I & $P^{* \mu}\left\langle f_{+} \nu \lambda\right\rangle f_{+\nu}^{\rho} D_{\lambda \rho} P^{* \dagger}{ }_{\mu}$ & 193 & & & \\
\hline$P\left\langle u^{\mu} u_{\mu} u^{\nu} u^{\lambda}\right\rangle D_{\nu \lambda} P^{\dagger}$ & & & 13 & I & $P^{* \mu}\left\langle f_{+\mu}{ }^{\nu} f_{+\nu}{ }^{\lambda}\right\rangle P^{* \dagger} \lambda$ & & & 342 & I \\
\hline$P\left\langle u^{\mu} u^{\nu} u_{\mu} u^{\lambda}\right\rangle D_{\nu \lambda} P^{\dagger}$ & & & 14 & I & $P^{* \mu}\left\langle f_{+}{ }^{\nu \lambda} f_{+\nu \lambda}\right\rangle P^{* \dagger}{ }_{\mu}$ & & & 343 & \\
\hline$P\left\langle u^{\mu} u^{\nu} u^{\lambda} u^{\rho}\right\rangle D_{\mu \nu \lambda \rho} P^{\dagger}$ & & & 15 & I & $P^{* \mu}\left\langle f_{+\mu}^{\nu} f_{+}^{\lambda \rho}\right\rangle D_{\nu \lambda} P^{* \dagger} \rho$ & & & 344 & I \\
\hline$P u^{\mu} u_{\mu} u^{\nu} u_{\nu} P^{\dagger}$ & & & 16 & I & $P^{* \mu}\left\langle f_{+}{ }^{\nu \lambda} f_{+\nu}{ }^{\rho}\right\rangle D_{\lambda \rho} P^{* \dagger}{ }_{\mu}$ & & & 345 & \\
\hline$P u^{\mu} u^{\nu} u_{\mu} u_{\nu} P^{\dagger}$ & & & 17 & I & $P^{* \mu} u_{\mu} u^{\nu} \chi_{+} P^{* \dagger}{ }_{\nu}+$ H.c. & 194 & I & 346 & I \\
\hline$P u^{\mu} u_{\mu} u^{\nu} u^{\lambda} D_{\nu \lambda} P^{\dagger}+$ H.c. & & & 18 & I & $P^{* \mu} u^{\nu} u_{\mu} \chi_{+} P_{\nu}^{* \dagger}+$ H.c. & 195 & & 347 & \\
\hline$P u^{\mu} u^{\nu} u_{\mu} u^{\lambda} D_{\nu \lambda} P^{\dagger}+$ H.c. & & & 19 & I & $P^{* \mu} u^{\nu} u_{\nu} \chi_{+} P^{* \dagger}{ }_{\mu}+$ H.c. & 196 & & 348 & \\
\hline$\varepsilon^{\mu \nu \lambda \rho} P u_{\mu} u_{\nu} f_{-\lambda \rho} P^{\dagger}+$ H.c. & 6 & I & 20 & I & $P^{* \mu} u_{\mu} \chi_{+} u^{\nu} P^{* \dagger}{ }_{\nu}$ & 197 & I & 349 & I \\
\hline$\varepsilon^{\mu \nu \lambda \rho} P u_{\mu} u_{\nu} f_{-\lambda}{ }^{\sigma} D_{\rho \sigma} P^{\dagger}+$ H.c. & 7 & I & 21 & I & $P^{* \mu} u^{\nu} \chi+u_{\mu} P^{* \dagger}{ }_{\nu}$ & 198 & & 350 & \\
\hline$\varepsilon^{\mu \nu \lambda \rho} P u_{\mu} u^{\sigma} f_{-\nu \lambda} D_{\rho \sigma} P^{\dagger}+$ H.c. & 8 & I & 22 & I & $P^{* \mu} u^{\nu} \chi_{+} u_{\nu} P^{* \dagger}{ }_{\mu}$ & 199 & & 351 & \\
\hline$\varepsilon^{\mu \nu \lambda \rho} P u_{\mu} f_{-\nu \lambda} u^{\sigma} D_{\rho \sigma} P^{\dagger}+$ H.c. & 9 & I & 23 & I & $P^{* \mu} u^{\nu} u^{\lambda} \chi_{+} D_{\nu \lambda} P^{* \dagger}{ }_{\mu}+$ H.c. & 200 & & 352 & \\
\hline$\varepsilon^{\mu \nu \lambda \rho} P u_{\mu} u_{\nu} h_{\lambda}{ }^{\sigma} D_{\rho \sigma} P^{\dagger}+$ H.c. & 10 & I & 24 & I & $P^{* \mu} u^{\nu} \chi_{+} u^{\lambda} D_{\nu \lambda} P_{\mu}^{* \dagger}$ & 201 & & 353 & \\
\hline$\varepsilon^{\mu \nu \lambda \rho} P\left\langle u_{\mu} f_{-\nu \lambda}\right\rangle u_{\rho} P^{\dagger}$ & & & 25 & I & $P^{* \mu}\left\langle u_{\mu} u^{\nu}\right\rangle \chi_{+} P_{\nu}^{* \dagger}$ & & & 354 & I \\
\hline$\varepsilon^{\mu \nu \lambda \rho} P\left\langle u_{\mu} f_{-\nu}{ }^{\sigma}\right\rangle u_{\lambda} D_{\rho \sigma} P^{\dagger}$ & & & 26 & I & $P^{* \mu}\left\langle u_{\mu} u^{\nu} \chi_{+}\right\rangle P_{\nu}^{* \dagger}$ & 202 & I & 355 & I \\
\hline$\varepsilon^{\mu \nu \lambda \rho} P\left\langle u_{\mu} u^{\sigma}\right\rangle f_{-\nu \lambda} D_{\rho \sigma} P^{\dagger}$ & & & 27 & I & $P^{* \mu}\left\langle u^{\nu} \chi_{+}\right\rangle u_{\mu} P^{* \dagger}{ }_{\nu}+$ H.c. & & & 356 & I \\
\hline$\varepsilon^{\mu \nu \lambda \rho} P\left\langle u_{\mu} u^{\sigma} f_{-\nu \lambda}\right\rangle D_{\rho \sigma} P^{\dagger}+$ H.c. & & & 28 & I & $P^{* \mu}\left\langle\chi_{+}\right\rangle u_{\mu} u^{\nu} P^{* \dagger}{ }_{\nu}$ & & & 357 & I \\
\hline$\varepsilon^{\mu \nu \lambda \rho} P\left\langle u_{\mu} h_{\nu}{ }^{\sigma}\right\rangle u_{\lambda} D_{\rho \sigma} P^{\dagger}$ & & & 29 & I & $P^{* \mu}\left\langle u^{\nu} u_{\mu} \chi_{+}\right\rangle P^{* \dagger}{ }_{\nu}$ & & & 358 & \\
\hline$P f_{-}^{\mu \nu} f_{-\mu \nu}^{\mu} P^{\dagger}$ & & & 30 & I & $P^{* \mu}\left\langle\chi_{+}\right\rangle u^{\nu} u_{\mu} P^{* \dagger}{ }_{\nu}$ & & & 359 & \\
\hline$P f_{-}{ }^{\mu \nu} f_{-\mu}{ }^{\lambda} D_{\nu \lambda} P^{\dagger}$ & & & 31 & I & $P^{* \mu}\left\langle u^{\nu} u_{\nu}\right\rangle \chi_{+}^{\mu} P_{\mu \nu}^{* \dagger}$ & & & 360 & \\
\hline$P f_{-}{ }^{\mu \nu} h_{\mu}{ }^{\lambda} D_{\nu \lambda} P^{\dagger}+$ H.c. & 11 & I & 32 & I & $P^{* \mu}\left\langle u^{\nu} u_{\nu} \chi_{+}\right\rangle P^{* \dagger}{ }_{\mu}$ & 203 & & 361 & \\
\hline$P h^{\mu \nu} h_{\mu \nu} P^{\dagger}$ & 12 & I & 33 & I & $P^{* \mu}\left\langle u^{\nu} \chi_{+}\right\rangle u_{\nu} P_{\mu}^{* \dagger}$ & & & 362 & \\
\hline$P h^{\mu \nu} h_{\mu}{ }^{\lambda} D_{\nu \lambda} P^{\dagger}$ & 13 & I & 34 & I & $P^{* \mu}\left\langle\chi_{+}\right\rangle u^{\nu} u_{\nu} P_{\mu}^{* \dagger}$ & & & 363 & \\
\hline$P h^{\mu \nu} h^{\lambda \rho} D_{\mu \nu \lambda \rho} P^{\dagger}$ & 14 & I & 35 & I & $P^{* \mu}\left\langle u^{\nu} u^{\lambda}\right\rangle \chi_{+} D_{\nu \lambda} P^{* \dagger}{ }_{\mu}$ & & & 364 & \\
\hline$P u^{\mu} \nabla^{\nu} f_{-\mu \nu}^{\mu \nu} P^{\dagger}+$ H.c. & 15 & I & 36 & I & $P^{* \mu}\left\langle u^{\nu} u^{\lambda} \chi_{+}\right\rangle D_{\nu \lambda} P^{* \dagger}{ }_{\mu}^{\mu}$ & 204 & & 365 & \\
\hline$P u^{\mu} \nabla^{\nu} f_{-\mu}^{\lambda} D_{\nu \lambda} P^{\dagger}+$ H.c. & 16 & I & 37 & I & $P^{* \mu}\left\langle u^{\nu} \chi_{+}\right\rangle u^{\lambda} D_{\nu \lambda} P_{\mu}^{* \dagger}$ & & & 366 & \\
\hline$P u^{\mu} \nabla^{\nu} f_{-\nu}^{\lambda} D_{\mu \lambda} P^{\dagger}+$ H.c. & 17 & I & 38 & I & $P^{* \mu}\left\langle\chi_{+}\right\rangle u^{\nu} u^{\lambda} D_{\nu \lambda} P_{\mu \dagger}^{* \mu}$ & & & 367 & \\
\hline$P u^{\mu} \nabla_{\mu} h^{\nu \lambda} D_{\nu \lambda} P^{\dagger}+$ H.c. & 18 & I & 39 & I & $\varepsilon^{\mu \nu \lambda \rho} P_{\mu}^{*} f_{-\nu \lambda} \chi_{+} P^{* \dagger}{ }_{\rho}^{\mu}+$ H.c. & 205 & I & 368 & I \\
\hline$P u^{\mu} \nabla^{\nu} h^{\lambda \rho} D_{\mu \nu \lambda \rho} P^{\dagger}+$ H.c. & 19 & I & 40 & I & $\varepsilon^{\mu \nu \lambda \rho} P^{*}{ }_{\mu} h_{\nu}{ }^{\sigma} \chi_{+} D_{\lambda \sigma} P_{\rho}^{* \dagger} \rho+$ H.c. & 206 & I & 369 & I \\
\hline$P\left\langle f_{-}{ }^{\mu \nu} h_{\mu}{ }^{\lambda}\right\rangle D_{\nu \lambda} P^{\dagger}$ & & & 41 & I & $\varepsilon^{\mu \nu \lambda \rho} P_{\mu}^{*} u_{\nu} \nabla_{\lambda} \chi_{+} P_{\rho}^{* \dagger}+$ H.c. & 207 & I & 370 & I \\
\hline$P\left\langle h^{\mu \nu} h_{\mu \nu}^{\mu}\right\rangle P^{\dagger}$ & & & 42 & I & $\varepsilon^{\mu \nu \lambda \rho} P_{\mu}^{*}{ }_{\mu} u_{\nu} \nabla^{\sigma} \chi_{+} D_{\lambda \sigma} P^{* \dagger}{ }_{\rho}+$ H.c. & 208 & I & 371 & I \\
\hline$P\left\langle h^{\mu \nu} h_{\mu}{ }^{\lambda}\right\rangle D_{\nu \lambda} P^{\dagger}$ & & & 43 & I & $P^{* \mu} \nabla_{\mu} \nabla^{\mu} \chi_{+} P_{\nu}^{* \dagger}$ & 209 & I & 372 & I \\
\hline$P\left\langle h^{\mu \nu} h^{\lambda \rho}\right\rangle D_{\mu \nu \lambda \rho} P^{\dagger}$ & & & 44 & I & $P^{* \mu} \nabla^{\nu} \nabla_{\nu} \chi_{+} P_{\mu}^{* \dagger}$ & 210 & & 373 & \\
\hline$P\left\langle u^{\mu} \nabla^{\nu} f_{-\mu \nu}\right\rangle P^{\dagger}$ & & & 45 & I & $P^{* \mu} \nabla^{\nu} \nabla^{\lambda} \chi_{+} D_{\nu \lambda} P^{* \dagger}{ }_{\mu}$ & 211 & & 374 & \\
\hline$P\left\langle u^{\mu} \nabla^{\nu} f_{-\mu}{ }^{\lambda}\right\rangle D_{\nu \lambda} P^{\dagger}$ & & & 46 & I & $P^{* \mu}\left\langle\nabla_{\mu} \nabla^{\nu} \chi_{+}\right\rangle P_{\nu}^{* \dagger}$ & 212 & I & 375 & I \\
\hline
\end{tabular}


TABLE IX. (Continued)

\begin{tabular}{|c|c|c|c|c|c|c|c|c|c|}
\hline$O_{n}$ & $S U(2)$ & $\tilde{c}_{n}^{(4)}$ & $S U(3)$ & $\tilde{C}_{n}^{(4)}$ & $O_{n}$ & $S U(2)$ & $\tilde{c}_{n}^{(4)}$ & $S U(3)$ & $\tilde{C}_{n}^{(4)}$ \\
\hline$P\left\langle u^{\mu} \nabla^{\nu} f_{-\nu}{ }^{\lambda}\right\rangle D_{\mu \lambda} P^{\dagger}$ & & & 47 & I & $P^{* \mu}\left\langle\nabla^{\nu} \nabla_{\nu} \chi_{+}\right\rangle P_{\mu}^{* \dagger}$ & 213 & & 376 & \\
\hline$P\left\langle u^{\mu} \nabla_{\mu} h^{\nu \lambda}\right\rangle D_{\nu \lambda} P^{\dagger}$ & & & 48 & I & $P^{* \mu}\left\langle\nabla^{\nu} \nabla^{\lambda} \chi_{+}\right\rangle D_{\nu \lambda} P_{\mu}^{* \dagger}$ & 214 & & 377 & \\
\hline$P\left\langle u^{\mu} \nabla^{\nu} h^{\lambda \rho}\right\rangle D_{\mu \nu \lambda \rho} P^{\dagger}$ & & & 49 & I & $i P^{* \mu} f_{+\mu}{ }^{\nu} \chi_{+} P^{* \dagger}{ }_{\nu}+$ H.c. & 215 & I & 378 & I \\
\hline$i P f_{+}{ }^{\mu \nu} u_{\mu} u_{\nu} P^{\dagger}+$ H.c. & 20 & I & 50 & I & $i P^{* \mu}\left\langle f_{+\mu}{ }^{\nu}\right\rangle \chi_{+} P_{\nu}^{* \dagger}$ & 216 & I & & \\
\hline$i P u^{\mu} f_{+\mu}{ }^{\mu} u_{\nu} P^{\dagger}$ & 21 & I & 51 & I & $i P^{* \mu}\left\langle f_{+\mu}{ }^{\nu} \chi_{+}\right\rangle P^{* \dagger}{ }_{\nu}$ & 217 & I & 379 & I \\
\hline$i P f_{+}{ }^{\mu \nu} u_{\mu} u^{\lambda} D_{\nu \lambda} P^{\dagger}+$ H.c. & 22 & I & 52 & I & $i P^{* \mu}\left\langle\chi_{+}\right\rangle f_{+\mu}{ }^{\nu} P^{* \dagger}{ }_{\nu}$ & 218 & I & 380 & I \\
\hline$i P f_{+}{ }^{\mu \nu} u^{\lambda} u_{\mu} D_{\nu \lambda} P^{\dagger}+$ H.c. & & & 53 & I & $P^{* \mu} \chi_{+}^{2} P_{\mu}^{* \dagger}$ & 219 & & 381 & \\
\hline$i P u^{\mu} f_{+\mu}{ }^{\nu} u^{\lambda} D_{\nu \lambda} P^{\dagger}+$ H.c. & 23 & I & 54 & I & $P^{* \mu}\left\langle\chi_{+}\right\rangle \chi_{+} P^{* \dagger}{ }_{\mu}$ & & & 382 & \\
\hline$i P\left\langle f_{+}{ }^{\mu \nu} u_{\mu} u_{\nu}\right\rangle P^{\dagger}$ & & & 55 & I & $P^{* \mu}\left\langle\chi_{+}^{2}\right\rangle P_{\mu}^{* \dagger}$ & 220 & & 383 & \\
\hline$i P\left\langle f_{+}{ }^{\mu \nu} u_{\mu} u^{\lambda}\right\rangle D_{\nu \lambda} P^{\dagger}+$ H.c. & & & 56 & I & $P^{* \mu}\left\langle\chi_{+}\right\rangle\left\langle\chi_{+}\right\rangle P_{\mu}^{* \dagger}$ & & & 384 & \\
\hline$i \varepsilon^{\mu \nu \lambda \rho} P f_{+\mu \nu} f_{-\lambda \rho} P^{\dagger}+$ H.c. & 24 & I & 57 & I & $i \varepsilon^{\mu \nu \lambda \rho} P_{\mu}^{*} u_{\nu} u_{\lambda} \chi_{-} P^{* \dagger}{ }_{\rho}+$ H.c. & 221 & I & 385 & I \\
\hline$i \varepsilon^{\mu \nu \lambda \rho} P f_{+\mu \nu} f_{-\lambda}^{\sigma} D_{\rho \sigma} P^{\dagger}+$ H.c. & 25 & I & 58 & I & $i \varepsilon^{\mu \nu \lambda \rho} P_{\mu}^{*} u_{\nu} \chi_{-} u_{\lambda} P^{* \dagger} \rho$ & 222 & I & 386 & I \\
\hline$i \varepsilon^{\mu \nu \lambda \rho} P f_{+\mu \nu} h_{\lambda}^{\sigma} D_{\rho \sigma} P^{\dagger}+$ H.c. & 26 & I & 59 & I & $i \varepsilon^{\mu \nu \lambda \rho} P_{\mu}^{*} u_{\nu} u^{\sigma} \chi_{-} D_{\lambda \sigma} P^{* \dagger}{ }_{\rho}^{*}+$ H.c. & & & 387 & I \\
\hline$i \varepsilon^{\mu \nu \lambda \rho} P \nabla_{\mu} f_{+\nu}{ }^{\sigma} u_{\lambda} D_{\rho \sigma} P^{\dagger}+$ H.c. & 27 & I & 60 & I & $i \varepsilon^{\mu \nu \lambda \rho} P_{\mu}^{*}\left\langle u_{\nu} u_{\lambda} \chi_{-}\right\rangle P_{\rho}^{* \dagger}$ & & & 388 & I \\
\hline$P f_{+}{ }^{\mu \nu} f_{+\mu \nu} P^{\dagger}$ & 28 & I & 61 & I & $i \varepsilon^{\mu \nu \lambda \rho} P^{*}{ }_{\mu}\left\langle\chi_{-}\right\rangle u_{\nu} u_{\lambda} P_{\rho}^{* \dagger}$ & & & 389 & I \\
\hline$P f_{+}{ }^{\mu \nu} f_{+\mu}^{\lambda} D_{\nu \lambda} P^{\dagger}$ & 29 & I & 62 & I & $i P^{* \mu} f_{-\mu}{ }^{\nu} \chi_{-} P^{* \dagger}{ }_{\nu}+$ H.c. & 223 & I & 390 & I \\
\hline$P\left\langle f_{+}{ }^{\mu \nu}\right\rangle f_{+\mu \nu} P^{\dagger}$ & 30 & I & & & $i P^{* \mu} h_{\mu}^{\nu} \chi_{-} P_{\nu}^{* \dagger}+$ H.c. & 224 & I & 391 & I \\
\hline$P\left\langle f_{+}{ }^{\mu \nu}\right\rangle f_{+\mu}{ }^{\lambda} D_{\nu \lambda} P^{\dagger}$ & 31 & I & & & $i P^{* \mu} h^{\nu \lambda} \chi_{-} D_{\nu \lambda} P^{* \dagger}{ }_{\mu}+$ H.c. & 225 & & 392 & \\
\hline$P\left\langle f_{+}^{\mu \nu} f_{+\mu \nu}\right\rangle P^{\dagger}$ & & & 63 & I & $i P^{* \mu} u_{\mu} \nabla^{\nu} \chi_{-} P_{\nu}^{* \dagger}{ }_{\nu}^{\mu}+$ H.c. & 226 & I & 393 & I \\
\hline$P\left\langle f_{+}{ }^{\mu \nu} f_{+\mu}{ }^{\lambda}\right\rangle D_{\nu \lambda} P^{\dagger}$ & & & 64 & I & $i P^{* \mu} u^{\nu} \nabla_{\mu} \chi_{-} P^{* \dagger}{ }_{\nu}+$ H.c. & 227 & & 394 & \\
\hline$P u^{\mu} u_{\mu} \chi_{+} P^{\dagger}+$ H.c. & 32 & I & 65 & I & $i P^{* \mu} u^{\nu} \nabla_{\nu} \chi_{-} P_{\mu}^{* \dagger}+$ H.c. & 228 & & 395 & \\
\hline$P u^{\mu} \chi_{+} u_{\mu} P^{\dagger}$ & 33 & I & 66 & I & $i P^{* \mu} u^{\nu} \nabla^{\lambda} \chi_{-} D_{\nu \lambda} P^{* \dagger}{ }_{\mu}+$ H.c. & 229 & & 396 & \\
\hline$P u^{\mu} u^{\nu} \chi_{+} D_{\mu \nu} P^{\dagger}+$ H.c. & 34 & I & 67 & I & $i P^{* \mu}\left\langle h_{\mu}{ }^{\nu} \chi_{-}\right\rangle P^{* \dagger}{ }_{\nu}$ & 230 & I & 397 & I \\
\hline$P u^{\mu} \chi_{+} u^{\nu} D_{\mu \nu} P^{\dagger}$ & 35 & I & 68 & I & $i P^{* \mu}\left\langle\chi_{-}\right\rangle h_{\mu}{ }^{\nu} P_{\nu}^{* \dagger}$ & & & 398 & I \\
\hline$P\left\langle u^{\mu} u_{\mu}\right\rangle \chi_{+} P^{\dagger}$ & & & 69 & I & $i P^{* \mu}\left\langle h^{\nu \lambda} \chi_{-}\right\rangle D_{\nu \lambda} P^{* \dagger}{ }_{\mu}$ & 231 & & 399 & \\
\hline$P\left\langle u^{\mu} u_{\mu} \chi_{+}\right\rangle P^{\dagger}$ & 36 & I & 70 & I & $i P^{* \mu}\left\langle\chi_{-}\right\rangle h^{\nu \lambda} D_{\nu \lambda} P_{\mu}^{* \dagger}$ & & & 400 & \\
\hline$P\left\langle u^{\mu} \chi_{+}\right\rangle u_{\mu} P^{\dagger}$ & & & 71 & I & $i P^{* \mu}\left\langle u_{\mu} \nabla^{\nu} \chi_{-}\right\rangle P^{* \dagger}{ }_{\nu}+$ H.c. & 232 & I & 401 & I \\
\hline$P\left\langle\chi_{+}\right\rangle u^{\mu} u_{\mu} P^{\dagger}$ & & & 72 & I & $i P^{* \mu}\left\langle\nabla^{\nu} \chi_{-}\right\rangle u_{\mu} P^{* \dagger}{ }_{\nu}+$ H.c. & & & 402 & I \\
\hline$P\left\langle u^{\mu} u^{\nu}\right\rangle \chi_{+} D_{\mu \nu} P^{\dagger}$ & & & 73 & I & $i P^{* \mu}\left\langle u^{\nu} \nabla_{\nu} \chi_{-}\right\rangle P^{* \dagger}{ }_{\mu}$ & 233 & & 403 & \\
\hline$P\left\langle u^{\mu} u^{\nu} \chi_{+}\right\rangle D_{\mu \nu} P^{\dagger}$ & 37 & I & 74 & I & $i P^{* \mu}\left\langle\nabla^{\nu} \chi_{-}\right\rangle u_{\nu} P^{* \dagger}{ }_{\mu}^{\mu}$ & & & 404 & \\
\hline$P\left\langle u^{\mu} \chi_{+}\right\rangle u^{\nu} D_{\mu \nu} P^{\dagger}$ & & & 75 & I & $i P^{* \mu}\left\langle u^{\nu} \nabla^{\lambda} \chi_{-}\right\rangle D_{\nu \lambda} P^{* \dagger}{ }_{\mu}$ & 234 & & 405 & \\
\hline$P\left\langle\chi_{+}\right\rangle u^{\mu} u^{\nu} D_{\mu \nu} P^{\dagger}$ & & & 76 & I & $i P^{* \mu}\left\langle\nabla^{\nu} \chi_{-}\right\rangle u^{\lambda} D_{\nu \lambda} P^{* \dagger}{ }_{\mu}$ & & & 406 & \\
\hline$P \nabla^{\mu} \nabla_{\mu} \chi_{+} P^{\dagger}$ & 38 & I & 77 & I & $\varepsilon^{\mu \nu \lambda \rho} P_{\mu}^{*} f_{+\nu \lambda} \chi_{-} P^{* \dagger} \rho+$ H.c. & 235 & I & 407 & I \\
\hline$P \nabla^{\mu} \nabla^{\nu} \chi_{+} D_{\mu \nu} P^{\dagger}$ & 39 & I & 78 & I & $\varepsilon^{\mu \nu \lambda \rho} P_{\mu}^{*}\left\langle f_{+\nu \lambda}\right\rangle \chi_{-} P^{* \dagger} \rho$ & 236 & I & & \\
\hline$P\left\langle\nabla^{\mu} \nabla_{\mu} \chi_{+}\right\rangle P^{\dagger}$ & 40 & I & 79 & I & $\varepsilon^{\mu \nu \lambda \rho} P^{*}{ }_{\mu}\left\langle f_{+\nu \lambda \chi_{-}}\right\rangle P^{* \dagger} \rho$ & 237 & I & 408 & I \\
\hline$P\left\langle\nabla^{\mu} \nabla^{\nu} \chi_{+}\right\rangle D_{\mu \nu} P^{\dagger}$ & 41 & I & 80 & I & $\varepsilon^{\mu \nu \lambda \rho} P_{\mu}^{*}\left\langle\chi_{-}\right\rangle f_{+\nu \lambda} P^{* \dagger}{ }_{\rho}^{*}$ & 238 & I & 409 & I \\
\hline$P \chi_{+}^{2} P^{\dagger}$ & 42 & I & 81 & I & $P^{* \mu} \chi_{-}^{2} P_{\mu}^{* \dagger}$ & 239 & & 410 & \\
\hline$P\left(\chi_{+}\right\rangle \chi_{+} P^{\dagger}$ & & & 82 & I & $P^{* \mu}\left\langle\chi_{-}\right\rangle \chi_{-}^{\mu} P_{\mu}^{* \dagger}$ & 240 & & 411 & \\
\hline$P\left\langle\chi_{+}^{2}\right\rangle P^{\dagger}$ & 43 & I & 83 & I & $P^{* \mu}\left\langle\chi_{-}\right\rangle\left\langle\chi_{-}\right\rangle P_{\mu}^{* \dagger}$ & & & 412 & \\
\hline$P\left\langle\chi_{+}\right\rangle\left\langle\chi_{+}\right\rangle P^{\dagger}$ & & & 84 & I & $i P^{* \mu}\left\langle D_{\mu} D^{\nu} F_{L \nu}^{\lambda}\right\rangle P_{\lambda}^{* \dagger}+P .+$ H.c. & 241 & I & & \\
\hline$i P h^{\mu \nu} \chi_{-} D_{\mu \nu} P^{\dagger}+$ H.c. & 44 & I & 85 & I & $i P^{* \mu}\left\langle D_{\mu} D^{\nu} F_{L}^{\lambda \rho}\right\rangle D_{\nu \lambda} P^{* \dagger} \rho+P .+$ H.c. & 242 & I & & \\
\hline$i P u^{\mu} \nabla_{\mu} \chi_{-} P^{\dagger}+$ H.c. & 45 & I & 86 & I & $P^{* \mu}\left\langle F_{L \mu}^{\nu} F_{L \nu}^{\lambda}\right\rangle P_{\lambda}^{* \dagger}+$ H.c. & 243 & & 413 & I \\
\hline$i P u^{\mu} \nabla^{\nu} \chi_{-} D_{\mu \nu} P^{\dagger}+$ H.c. & 46 & I & 87 & I & $P^{* \mu}\left\langle F_{L}^{\nu \lambda} F_{L \nu \lambda}\right\rangle P^{* \dagger}{ }_{\mu}+$ H.c. & 244 & & 414 & \\
\hline$i P\left\langle h^{\mu \nu} \chi_{-}\right\rangle D_{\mu \nu} P^{\dagger}$ & 47 & I & 88 & I & $P^{* \mu}\left\langle F_{L \mu}{ }^{\nu} F_{L}^{\lambda \rho}\right\rangle D_{\nu \lambda} P_{\rho}^{* \dagger}+$ H.c. & 245 & & 415 & I \\
\hline$i P\left\langle\chi_{-}\right\rangle h^{\mu \nu} D_{\mu \nu} P^{\dagger}$ & & & 89 & I & $P^{* \mu}\left\langle F_{L}{ }^{\nu \lambda} F_{L \nu}{ }^{\rho}\right\rangle D_{\lambda \rho} P_{\mu}^{* \dagger}+$ H.c. & 246 & & 416 & \\
\hline$i P\left\langle u^{\mu} \nabla_{\mu} \chi_{-}\right\rangle P^{\dagger}$ & 48 & I & 90 & I & $P^{* \mu}\left\langle F_{L \mu}{ }^{\nu}\right\rangle\left\langle F_{L \nu}{ }^{\lambda}\right\rangle P^{* \dagger} \lambda+$ H.c. & 247 & & & \\
\hline$i P\left\langle\nabla^{\mu} \chi_{-}\right\rangle u_{\mu} P^{\dagger}$ & & & 91 & I & $P^{* \mu}\left\langle F_{L}{ }^{\nu \lambda}\right\rangle\left\langle F_{L \nu \lambda}\right\rangle P^{* \dagger}{ }_{\mu}+$ H.c. & 248 & & & \\
\hline$i P\left\langle u^{\mu} \nabla^{\nu} \chi_{-}\right\rangle D_{\mu \nu} P^{\dagger}$ & 49 & I & 92 & I & $P^{* \mu}\left\langle F_{L \mu}{ }^{\nu}\right\rangle\left\langle F_{L}^{\lambda \rho}\right\rangle D_{\nu \lambda} P_{\rho}^{* \dagger}+$ H.c. & 249 & & & \\
\hline
\end{tabular}




\begin{tabular}{|c|c|c|c|c|c|c|c|c|c|}
\hline$O_{n}$ & $S U(2)$ & $\tilde{c}_{n}^{(4)}$ & $S U(3)$ & $\tilde{C}_{n}^{(4)}$ & $O_{n}$ & $S U(2)$ & $\tilde{c}_{n}^{(4)}$ & $S U(3)$ & $\tilde{C}_{n}^{(4)}$ \\
\hline$i P\left\langle\nabla^{\mu} \chi_{-}\right\rangle u^{\nu} D_{\mu \nu} P^{\dagger}$ & & & 93 & I & $P^{* \mu}\left\langle F_{L}^{\nu \lambda}\right\rangle\left\langle F_{L \nu}^{\rho}\right\rangle D_{\lambda \rho} P^{* \dagger}{ }_{\mu}+$ H.c. & 250 & & & \\
\hline$P \chi_{-}^{2} P^{\dagger}$ & 50 & I & 94 & I & $i \varepsilon^{\mu \nu \lambda \rho} P^{*}{ }_{\mu}\left\langle F_{L \nu \lambda} F_{L}{ }^{\sigma \delta}\right\rangle D_{\rho \sigma} P^{* \dagger}{ }_{\delta}+P .+$ H.c. & 251 & I & 417 & I \\
\hline$P\left(\chi_{-}\right) \chi_{-} P^{\dagger}$ & 51 & I & 95 & I & $P^{* \mu}\left\langle\chi \chi^{\dagger}\right\rangle P_{\mu}^{* \dagger}$ & 252 & & 418 & \\
\hline$P\left\langle\chi_{-}\right\rangle\left\langle\chi_{-}\right\rangle P^{\dagger}$ & & & 96 & I & $P^{* \mu} \operatorname{det} \chi P^{* \dagger}{ }_{\mu}+$ H.c. & 253 & & & \\
\hline$P\left\langle F_{L}^{\mu \nu} F_{L \mu \nu}\right\rangle P^{\dagger}+$ H.c. & 52 & I & 97 & I & $\varepsilon^{\mu \nu \lambda \rho} P u_{\mu} u_{\nu} u_{\lambda} u^{\sigma} D_{\rho} P_{\sigma}^{* \dagger}+$ H.c. & 254 & & 419 & \\
\hline$P\left\langle F_{L}{ }^{\mu \nu} F_{L \mu}{ }^{\lambda}\right\rangle D_{\nu \lambda} P^{\dagger}+$ H.c. & 53 & I & 98 & I & $\varepsilon^{\mu \nu \lambda \rho} P u_{\mu} u_{\nu} u_{\lambda} u^{\sigma} D_{\sigma} P_{\rho}^{* \dagger}+$ H.c. & 255 & & 420 & \\
\hline$P\left\langle F_{L}^{\mu \nu}\right\rangle\left\langle F_{L \mu \nu}\right\rangle P^{\dagger}+$ H.c. & 54 & I & & & $\varepsilon^{\mu \nu \lambda \rho} P u_{\mu} u_{\nu} u^{\sigma} u_{\lambda} D_{\rho} P_{\sigma}^{* \dagger}+$ H.c. & & & 421 & \\
\hline$P\left\langle F_{L}{ }^{\mu \nu}\right\rangle\left\langle F_{L \mu}{ }^{\lambda}\right\rangle D_{\nu \lambda} P^{\dagger}+$ H.c. & 55 & I & & & $\varepsilon^{\mu \nu \lambda \rho} P u_{\mu} u_{\nu} u^{\sigma} u_{\lambda} D_{\sigma} P_{\rho \dagger}^{* \dagger}+$ H.c. & & & 422 & \\
\hline$P\left\langle\chi \chi^{\dagger}\right\rangle P^{\dagger}$ & 56 & I & 99 & I & $\varepsilon^{\mu \nu \lambda \rho} P u_{\mu} u_{\nu} u^{\sigma} u_{\sigma} D_{\lambda} P_{\rho \dagger}^{* \dagger}+$ H.c. & 256 & & 423 & \\
\hline$P \operatorname{det} \chi P^{\dagger}+$ H.c. & 57 & I & & & $\varepsilon^{\mu \nu \lambda \rho} P u_{\mu} u^{\sigma} u_{\nu} u_{\lambda} D_{\rho} P^{* \dagger}{ }_{\sigma}+$ H.c. & & & 424 & \\
\hline$P^{* \mu}\left\langle u_{\mu} u^{\nu}\right\rangle u_{\nu} u^{\lambda} P^{* \dagger}{ }_{\lambda}+$ H.c. & 58 & $\mathrm{I}$ & 100 & I & $\varepsilon^{\mu \nu \lambda \rho} P u_{\mu} u^{\sigma} u_{\nu} u_{\lambda} D_{\sigma} P_{\rho}^{* \dagger}+$ H.c. & & & 425 & \\
\hline$P^{* \mu}\left\langle u_{\mu} u^{\nu}\right\rangle u^{\lambda} u_{\nu} P_{\lambda}^{* \dagger}+$ H.c. & 59 & & 101 & I & $\varepsilon^{\mu \nu \lambda \rho} P u_{\mu} u^{\sigma} u_{\nu} u_{\sigma} D_{\lambda} P_{\rho \dagger}^{* \dagger}+$ H.c. & & & 426 & \\
\hline$P^{* \mu}\left\langle u_{\mu} u^{\nu}\right\rangle u^{\lambda} u_{\lambda} P_{\nu}^{* \dagger}$ & 60 & I & 102 & I & $\varepsilon^{\mu \nu \lambda \rho} P u_{\mu} u^{\sigma} u_{\sigma} u_{\nu} D_{\lambda} P_{\rho}^{* \dagger}+$ H.c. & & & 427 & \\
\hline$P^{* \mu}\left\langle u^{\nu} u_{\nu}\right\rangle u_{\mu} u_{\lambda}^{\lambda} P_{\lambda}^{* \dagger}$ & 61 & & 103 & $\mathrm{I}$ & $\varepsilon^{\mu \nu \lambda \rho} P u_{\mu} u_{\nu} u^{\sigma} u^{\delta} D_{\lambda \sigma \delta} P_{\rho}^{* \dagger}+$ H.c. & 257 & & 428 & \\
\hline$P^{* \mu}\left\langle u^{\nu} u_{\nu}\right\rangle u^{\lambda} u_{\mu} P_{\lambda}^{* \dagger}$ & & & 104 & & $\varepsilon^{\mu \nu \lambda \rho} P u_{\mu} u^{\sigma} u_{\nu} u^{\delta} D_{\lambda \sigma \delta} P_{\rho}^{* \dagger}+$ H.c. & & & 429 & \\
\hline$P^{* \mu}\left\langle u^{\nu} u_{\nu}\right\rangle u^{\lambda} u_{\lambda} P^{* \dagger}{ }_{\mu}$ & 62 & & 105 & & $\varepsilon^{\mu \nu \lambda \rho} P u_{\mu} u^{\sigma} u^{\delta} u_{\nu} D_{\lambda \sigma \delta} P^{* \dagger}{ }_{\rho}+$ H.c. & & & 430 & \\
\hline$P^{* \mu}\left\langle u^{\nu} u^{\lambda}\right\rangle u_{\nu} u_{\lambda} P^{* \dagger}{ }_{\mu}$ & 63 & & 106 & & $\varepsilon^{\mu \nu \lambda \rho} P\left\langle u_{\mu} u^{\sigma}\right\rangle u_{\nu} u_{\lambda} D_{\rho} P^{* \dagger}{ }_{\sigma}+$ H.c. & & & 431 & \\
\hline$P^{* \mu}\left\langle u_{\mu} u^{\nu}\right\rangle u^{\lambda} u^{\rho} D_{\nu \lambda} P_{\rho}^{* \dagger}+$ H.c. & 64 & I & 107 & I & $\varepsilon^{\mu \nu \lambda \rho} P\left\langle u_{\mu} u^{\sigma}\right\rangle u_{\nu} u_{\lambda} D_{\sigma} P^{* \dagger} \rho+$ H.c. & & & 432 & \\
\hline$P^{* \mu}\left\langle u_{\mu} u^{\nu}\right\rangle u^{\lambda} u^{\rho} D_{\nu \rho} P^{* \dagger}{ }_{\lambda}+$ H.c. & 65 & & 108 & I & $\varepsilon^{\mu \nu \lambda \rho} P\left\langle u_{\mu} u^{\sigma}\right\rangle u_{\nu} u_{\sigma} D_{\lambda} P^{* \dagger} \rho+$ H.c. & & & 433 & I \\
\hline$P^{* \mu}\left\langle u_{\mu} u^{\nu}\right\rangle u^{\lambda} u^{\rho} D_{\lambda \rho} P_{\nu}^{* \dagger}$ & 66 & I & 109 & I & $\varepsilon^{\mu \nu \lambda \rho} P\left\langle u_{\mu} u^{\sigma}\right\rangle u_{\nu} u^{\delta} D_{\lambda \sigma \delta} P_{\rho}^{* \dagger}+$ H.c. & & & 434 & I \\
\hline$P^{* \mu}\left\langle u^{\nu} u_{\nu}\right\rangle u^{\lambda} u^{\rho} D_{\lambda \rho} P_{\mu}^{* \dagger}{ }_{\mu}$ & 67 & & 110 & & $\varepsilon^{\mu \nu \lambda \rho} P\left\langle u_{\mu} u_{\nu} u_{\lambda}\right\rangle u^{\sigma} D_{\rho} P^{* \dagger}{ }_{\sigma}+$ H.c. & & & 435 & \\
\hline$P^{* \mu}\left\langle u^{\nu} u^{\lambda}\right\rangle u_{\mu} u^{\rho} D_{\nu \lambda} P^{* \dagger} \rho$ & 68 & & 111 & I & $\varepsilon^{\mu \nu \lambda \rho} P\left\langle u_{\mu} u_{\nu} u_{\lambda}\right\rangle u^{\sigma} D_{\sigma} P^{* \dagger} \rho+$ H.c. & & & 436 & \\
\hline$P^{* \mu}\left\langle u^{\nu} u^{\lambda}\right\rangle u_{\nu} u^{\rho} D_{\lambda \rho} P^{* \dagger}{ }_{\mu}+$ H.c. & 69 & & 112 & & $P\left\langle u^{\mu} u_{\mu}\right\rangle f_{-}^{\nu \lambda} D_{\nu} P_{\lambda}^{* \dagger}+$ H.c. & 258 & I & 437 & \\
\hline$P^{* \mu}\left\langle u^{\nu} u^{\lambda}\right\rangle u^{\rho} u_{\mu} D_{\nu \lambda} P^{* \dagger} \rho$ & & & 113 & & $P\left\langle u^{\mu} u^{\nu}\right\rangle f_{-\mu}^{\lambda} D_{\nu} P_{\lambda}^{* \dagger}+$ H.c. & 259 & I & 438 & \\
\hline$P^{* \mu}\left\langle u^{\nu} u^{\lambda}\right\rangle u^{\rho} u_{\rho} D_{\nu \lambda} P_{\mu}^{* \dagger}{ }_{\mu}$ & & & 114 & & $P\left\langle u^{\mu} u^{\nu}\right\rangle f_{-\mu}^{\lambda} D_{\lambda} P_{\nu}^{* \dagger}+$ H.c. & 260 & I & 439 & \\
\hline$P^{* \mu}\left\langle u^{\nu} u^{\lambda}\right\rangle u^{\rho} u^{\sigma} D_{\nu \lambda \rho \sigma} P^{* \dagger}{ }_{\mu}$ & 70 & & 115 & & $P\left\langle u^{\mu} u^{\nu}\right\rangle f_{-}^{\lambda \rho} D_{\mu \nu \lambda} P^{* \dagger} \rho+$ H.c. & 261 & I & 440 & \\
\hline$P^{* \mu}\left\langle u_{\mu} u^{\nu} u_{\nu}\right\rangle u^{\lambda} P_{\lambda}^{* \dagger}+$ H.c. & & & 116 & & $P\left\langle u^{\mu} u_{\mu}\right\rangle h^{\nu \lambda} D_{\nu} P_{\lambda}^{* \dagger}+$ H.c. & 262 & I & 441 & \\
\hline$P^{* \mu}\left\langle u_{\mu} u^{\nu} u^{\lambda}\right\rangle u_{\nu} P_{\lambda}^{* \dagger}$ & & & 117 & I & $P\left\langle u^{\mu} u^{\nu}\right\rangle h_{\mu}^{\lambda} D_{\nu} P^{* \dagger} \lambda+$ H.c. & 263 & I & 442 & \\
\hline$P^{* \mu}\left\langle u_{\mu} u^{\nu} u^{\lambda}\right\rangle u_{\lambda} P^{* \dagger}{ }_{\nu}$ & & & 118 & & $P\left\langle u^{\mu} u^{\nu}\right\rangle h_{\mu}^{\lambda} D_{\lambda} P^{* \dagger}{ }_{\nu}+$ H.c. & 264 & I & 443 & \\
\hline$P^{* \mu}\left\langle u^{\nu} u_{\nu} u^{\lambda}\right\rangle u_{\lambda} P_{\mu}^{* \dagger}$ & & & 119 & & $P\left\langle u^{\mu} u^{\nu}\right\rangle h^{\lambda \rho} D_{\mu \nu \lambda} P_{\rho}^{* \dagger}+$ H.c. & 265 & I & 444 & \\
\hline$P^{* \mu}\left\langle u_{\mu} u^{\nu} u^{\lambda}\right\rangle u^{\rho} D_{\nu \lambda} P_{\rho}^{* \dagger}+$ H.c. & & & 120 & & $P\left\langle u^{\mu} u^{\nu}\right\rangle h^{\lambda \rho} D_{\mu \lambda \rho} P^{* \dagger}{ }_{\nu}+$ H.c. & 266 & I & 445 & \\
\hline$P^{* \mu}\left\langle u_{\mu} u^{\nu} u^{\lambda}\right\rangle u^{\rho} D_{\nu \rho} P_{\lambda}^{* \dagger}$ & & & 121 & I & $P\left\langle u^{\mu} f_{-\mu}{ }^{\nu}\right\rangle u^{\lambda} D_{\lambda} P^{* \dagger \dagger}{ }_{\nu}+$ H.c. & 267 & I & 446 & \\
\hline$P^{* \mu}\left\langle u_{\mu} u^{\nu} u^{\lambda}\right\rangle u^{\rho} D_{\lambda \rho} P_{\nu}^{* \dagger}{ }_{\nu}$ & & & 122 & & $P\left\langle u^{\mu} f_{-\mu}{ }^{\nu}\right\rangle u^{\lambda} D_{\nu} P_{\lambda}^{* \dagger}+$ H.c. & 268 & I & 447 & \\
\hline$P^{* \mu}\left\langle u^{\nu} u_{\nu} u^{\lambda}\right\rangle u^{\rho} D_{\lambda \rho} P^{* \dagger}{ }_{\mu}$ & & & 123 & & $P\left\langle u^{\mu} f_{-}{ }^{\nu \lambda}\right\rangle u_{\mu} D_{\nu} P^{* \dagger} \lambda+$ H.c. & 269 & I & 448 & \\
\hline$P^{* \mu}\left\langle u^{\nu} u^{\lambda} u^{\rho}\right\rangle u_{\nu} D_{\lambda \rho} P_{\mu \dagger}^{* \dagger}$ & & & 124 & & $P\left\langle u^{\mu} f_{-} \nu \lambda\right\rangle u_{\nu} D_{\mu} P_{\lambda \dagger}^{* \dagger}+$ H.c. & 270 & I & 449 & \\
\hline$P^{* \mu}\left\langle u^{\nu} u^{\lambda} u^{\rho}\right\rangle u^{\sigma} D_{\nu \lambda \rho \sigma} P^{* \dagger}{ }_{\mu}$ & & & 125 & & $P\left\langle u^{\mu} f_{-}{ }^{\nu \lambda}\right\rangle u_{\nu} D_{\lambda} P_{\mu}^{* \dagger}{ }_{\mu}+$ H.c. & 271 & I & 450 & \\
\hline$P^{* \mu}\left\langle u_{\mu} u^{\nu} u_{\nu} u^{\lambda}\right\rangle P_{\lambda}^{* \dagger}$ & & & 126 & I & $P\left\langle u^{\mu} f_{-}{ }^{\nu \lambda}\right\rangle u^{\rho} D_{\mu \nu \rho} P_{\lambda}^{* \dagger}+$ H.c. & 272 & I & 451 & \\
\hline$P^{* \mu}\left\langle u_{\mu} u^{\nu} u^{\lambda} u_{\nu}\right\rangle P_{\lambda}^{* \dagger}$ & & & 127 & I & $P\left\langle u^{\mu} h_{\mu}{ }^{\nu}\right\rangle u^{\lambda} D_{\lambda} P_{\nu}^{* \dagger}{ }_{\nu}+$ H.c. & 273 & I & 452 & \\
\hline$P^{* \mu}\left\langle u_{\mu} u^{\nu} u^{\lambda} u_{\lambda}\right\rangle P^{* \dagger}{ }_{\nu}$ & & & 128 & & $P\left\langle u^{\mu} h_{\mu}{ }^{\nu}\right\rangle u^{\lambda} D_{\nu} P_{\lambda}^{* \dagger}+$ H.c. & 274 & I & 453 & \\
\hline$P^{* \mu}\left\langle u^{\nu} u_{\nu} u^{\lambda} u_{\lambda}\right\rangle P_{\mu}^{* \dagger}{ }_{\mu}$ & & & 129 & & $P\left\langle u^{\mu} h^{\nu \lambda}\right\rangle u_{\mu} D_{\nu} P_{\lambda}^{* \dagger}+$ H.c. & 275 & I & 454 & \\
\hline$P^{* \mu}\left\langle u^{\nu} u^{\lambda} u_{\nu} u_{\lambda}\right\rangle P_{\mu}^{* \dagger}$ & & & 130 & & $P\left\langle u^{\mu} h^{\nu \lambda}\right\rangle u_{\nu} D_{\mu} P_{\lambda}^{* \dagger}+$ H.c. & 276 & I & 455 & \\
\hline$P^{* \mu}\left\langle u_{\mu} u^{\nu} u^{\lambda} u^{\rho}\right\rangle D_{\nu \lambda} P_{\rho}^{* \dagger} \rho$ & & & 131 & I & $P\left\langle u^{\mu} h^{\nu \lambda}\right\rangle u_{\nu} D_{\lambda} P_{\mu}^{* \dagger}+$ H.c. & 277 & I & 456 & \\
\hline$P^{* \mu}\left\langle u_{\mu} u^{\nu} u^{\lambda} u^{\rho}\right\rangle D_{\nu \rho} P_{\lambda}^{* \dagger}$ & & & 132 & $\mathrm{I}$ & $P\left\langle u^{\mu} h^{\nu \lambda}\right\rangle u^{\rho} D_{\mu \nu \rho} P_{\lambda}^{* \dagger}+$ H.c. & 278 & I & 457 & \\
\hline$P^{* \mu}\left\langle u_{\mu} u^{\nu} u^{\lambda} u^{\rho}\right\rangle D_{\lambda \rho} P^{* \dagger}{ }_{\nu}$ & & & 133 & & $P\left\langle u^{\mu} h^{\nu \lambda}\right\rangle u^{\rho} D_{\mu \nu \lambda} P^{* \dagger}{ }_{\rho}+$ H.c. & 279 & I & 458 & \\
\hline$P^{* \mu}\left\langle u^{\nu} u_{\nu} u^{\lambda} u^{\rho}\right\rangle D_{\lambda \rho} P_{\mu \dagger}^{* \dagger}$ & & & 134 & & $P\left\langle u^{\mu} h^{\nu \lambda}\right\rangle u^{\rho} D_{\nu \lambda \rho} P_{\mu}^{* \dagger}+$ H.c. & 280 & $\mathrm{I}$ & 459 & \\
\hline$P^{* \mu}\left\langle u^{\nu} u^{\lambda} u_{\nu} u^{\rho}\right\rangle D_{\lambda \rho} P_{\mu}^{* \dagger}{ }_{\mu}$ & & & 135 & & $P\left\langle u^{\mu} u_{\mu} f_{-}{ }^{\nu \lambda}\right\rangle D_{\nu} P_{\lambda}^{* \dagger}+$ H.c. & & & 460 & \\
\hline$P^{* \mu}\left\langle u^{\nu} u^{\lambda} u^{\rho} u^{\sigma}\right\rangle D_{\nu \lambda \rho \sigma} P_{\mu}^{* \dagger}{ }_{\mu}$ & & & 136 & & $P\left\langle u^{\mu} u^{\nu} f_{-\mu}{ }^{\lambda}\right\rangle D_{\nu} P_{\lambda}^{* \dagger}{ }_{\lambda}+$ H.c. & 281 & & 461 & \\
\hline$P^{* \mu} u_{\mu} u^{\nu} u_{\nu} u^{\lambda} P_{\lambda}^{* \dagger}$ & & & 137 & & $P\left\langle u^{\mu} u^{\nu} f_{-\mu}{ }^{\lambda}\right\rangle D_{\lambda} P_{\nu}^{* \dagger}+$ H.c. & 282 & & 462 & \\
\hline
\end{tabular}




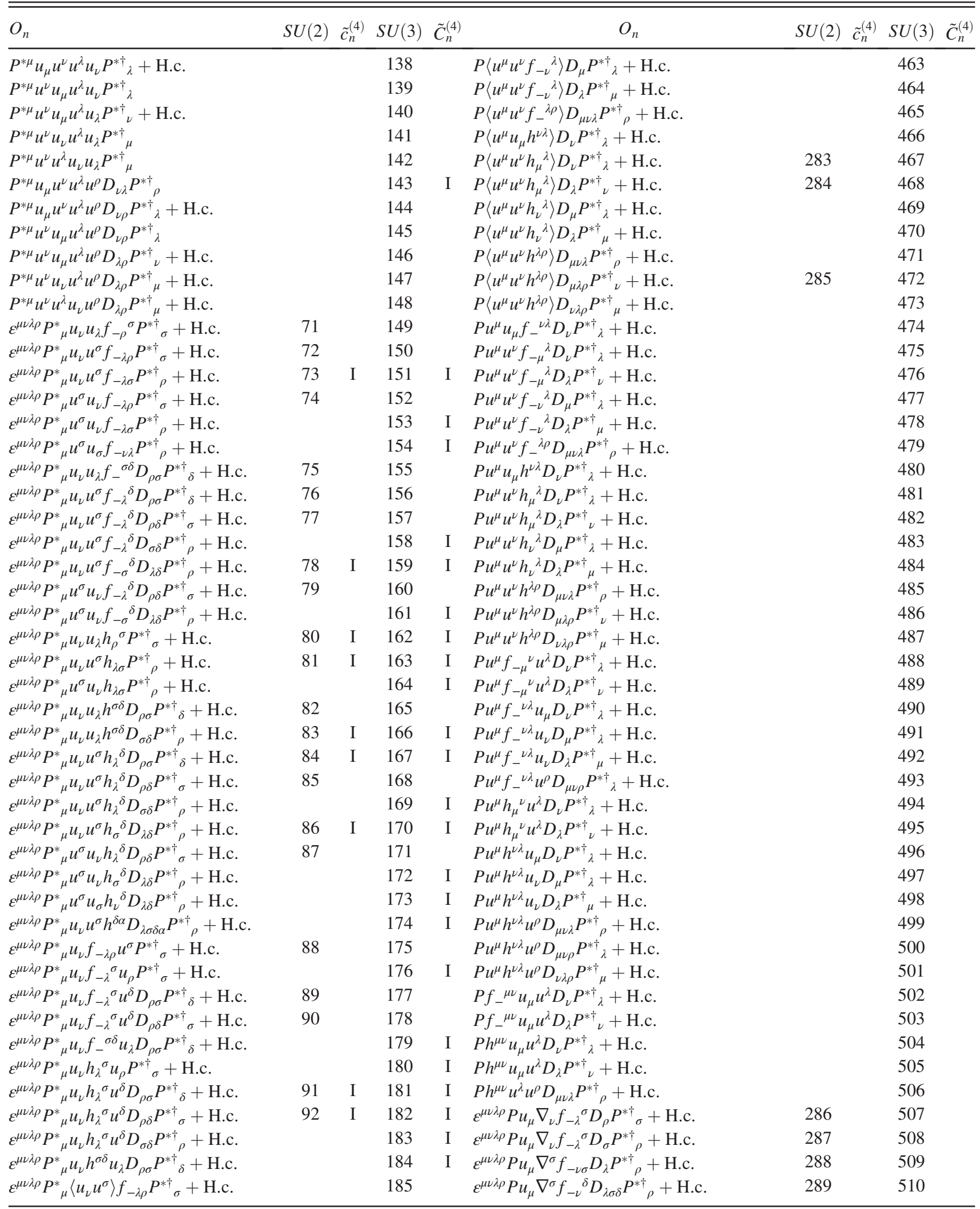




\begin{tabular}{|c|c|c|c|c|c|c|c|c|c|}
\hline$O_{n}$ & $S U(2)$ & $\tilde{c}_{n}^{(4)}$ & $S U(3)$ & $\tilde{C}_{n}^{(4)}$ & $O_{n}$ & $S U(2)$ & $\tilde{c}_{n}^{(4)}$ & $S U(3)$ & $\tilde{C}_{n}^{(4)}$ \\
\hline$\varepsilon^{\mu \nu \lambda \rho} P_{\mu}^{*}\left\langle u_{\nu} u^{\sigma}\right\rangle f_{-\lambda}{ }^{\delta} D_{\rho \sigma} P^{* \dagger}{ }_{\delta}+$ H.c. & & & 186 & & $\varepsilon^{\mu \nu \lambda \rho} P u_{\mu} \nabla_{\nu} h^{\sigma \delta} D_{\lambda \sigma \delta} P^{* \dagger}{ }_{\rho}+$ H.c. & 290 & & 511 & \\
\hline$\varepsilon^{\mu \nu \lambda \rho} P_{\mu}^{*}\left\langle u_{\nu} u^{\sigma}\right\rangle f_{-\lambda}{ }^{\delta} D_{\rho \delta} P^{* \dagger}{ }_{\sigma}+$ H.c. & & & 187 & & $\varepsilon^{\mu \nu \lambda \rho} P \nabla_{\mu} f_{-\nu}{ }^{\sigma} u_{\lambda} D_{\rho} P^{* \dagger}{ }_{\sigma}+$ H.c. & 291 & & 512 & \\
\hline$\varepsilon^{\mu \nu \lambda \rho} P_{\mu}^{*}\left\langle u_{\nu} u^{\sigma}\right\rangle h_{\lambda}{ }^{\delta} D_{\rho \sigma} P^{* \dagger}{ }_{\delta}+$ H.c. & & & 188 & I & $\varepsilon^{\mu \nu \lambda \rho} P \nabla_{\mu} f_{-\nu}{ }^{\sigma} u_{\lambda} D_{\sigma} P^{* \dagger}{ }_{\rho}+$ H.c. & 292 & & 513 & \\
\hline$\varepsilon^{\mu \nu \lambda \rho} P^{*}{ }_{\mu}\left\langle u_{\nu} u^{\sigma}\right\rangle h_{\lambda}{ }^{\delta} D_{\rho \delta} P^{* \dagger}{ }_{\sigma}+$ H.c. & & & 189 & $\mathrm{I}$ & $\varepsilon^{\mu \nu \lambda \rho} P \nabla_{\mu} f_{-\nu}{ }^{\sigma} u_{\sigma} D_{\lambda} P^{* \dagger}{ }_{\rho}+$ H.c. & 293 & & 514 & \\
\hline$\varepsilon^{\mu \nu \lambda \rho} P_{\mu}^{*}\left\langle u_{\nu} f_{-\lambda \rho}\right\rangle u^{\sigma} P_{\sigma}^{* \dagger}+$ H.c. & & & 190 & & $\varepsilon^{\mu \nu \lambda \rho} P \nabla_{\mu} f_{-\nu}{ }^{\sigma} u^{\delta} D_{\lambda \sigma \delta} P^{* \dagger}{ }_{\rho}+$ H.c. & 294 & & 515 & \\
\hline$\varepsilon^{\mu \nu \lambda \rho} P_{\mu}^{*}\left\langle u_{\nu} f_{-\lambda}{ }^{\sigma}\right\rangle u_{\rho} P_{\sigma}^{* \dagger}+$ H.c. & & & 191 & & $\varepsilon^{\mu \nu \lambda \rho} P f_{-\mu \nu} f_{-\lambda}{ }^{\sigma} D_{\rho} P_{\sigma}^{* \dagger}+$ H.c. & 295 & & 516 & \\
\hline$\varepsilon^{\mu \nu \lambda \rho} P_{\mu}^{*}\left\langle u^{\sigma} f_{-\nu \lambda}\right\rangle u_{\rho} P_{\sigma}^{* \dagger}+$ H.c. & & & 192 & & $\varepsilon^{\mu \nu \lambda \rho} P f_{-\mu \nu} f_{-\lambda}^{\sigma} D_{\sigma} P^{* \dagger} \rho+$ H.c. & 296 & & 517 & \\
\hline $\mathcal{E}^{\mu \nu \lambda \rho} P_{\mu}^{*}\left\langle u_{\nu} f_{-\lambda}{ }^{\sigma}\right\rangle u^{\delta} D_{\rho \delta} P^{* \dagger}{ }_{\sigma}+$ H.c. & & & 193 & & $\varepsilon^{\mu \nu \lambda \rho} P f_{-\mu \nu} h_{\lambda}{ }^{\sigma} D_{\rho} P^{* \dagger}{ }_{\sigma}+$ H.c. & 297 & & 518 & \\
\hline$\varepsilon^{\mu \nu \lambda \rho} P^{*}{ }_{\mu}\left\langle u_{\nu} f_{-\lambda} \sigma\right\rangle u^{\delta} D_{\rho \sigma} P^{* \dagger}{ }_{\delta}+$ H.c. & & & 194 & & $\varepsilon^{\mu \nu \lambda \rho} P f_{-\mu \nu} h_{\lambda}{ }^{\sigma} D_{\sigma} P_{\rho}^{* \dagger}+$ H.c. & 298 & & 519 & \\
\hline$\varepsilon^{\mu \nu \lambda \rho} P_{\mu}^{*}\left\langle u_{\nu} f_{-} \sigma \delta\right\rangle u_{\lambda} D_{\rho \sigma} P^{* \dagger}{ }_{\delta}+$ H.c. & & & 195 & & $\varepsilon^{\mu \nu \lambda \rho} P f_{-\mu \nu} h^{\sigma \delta} D_{\lambda \sigma \delta} P_{\rho}^{* \dagger}+$ H.c. & 299 & & 520 & \\
\hline$\varepsilon^{\mu \nu \lambda \rho} P_{\mu}^{*}\left\langle u^{\sigma} f_{-\nu \lambda}\right\rangle u^{\delta} D_{\rho \delta} P^{* \dagger}{ }_{\sigma}+$ H.c. & & & 196 & & $\varepsilon^{\mu \nu \lambda \rho} P \nabla_{\mu} h^{\sigma \delta} u_{\nu} D_{\lambda \sigma \delta} P^{* \dagger} \rho+$ H.c. & 300 & & 521 & \\
\hline$\varepsilon^{\mu \nu \lambda \rho} P_{\mu}^{*}\left\langle u_{\nu} h_{\lambda}{ }^{\sigma}\right\rangle u_{\rho} P^{* \dagger}{ }_{\sigma}+$ H.c. & & & 197 & I & $\varepsilon^{\mu \nu \lambda \rho} P h_{\mu}{ }^{\sigma} f_{-\nu \lambda} D_{\rho} P^{* \dagger}{ }_{\sigma}+$ H.c. & 301 & & 522 & \\
\hline$\varepsilon^{\mu \nu \lambda \rho} P_{\mu}^{*}\left\langle u_{\nu} h_{\lambda}{ }^{\sigma}\right\rangle u^{\delta} D_{\rho \delta} P^{* \dagger}{ }_{\sigma}+$ H.c. & & & 198 & I & $\varepsilon^{\mu \nu \lambda \rho} P h_{\mu}{ }^{\sigma} f_{-\nu \lambda} D_{\sigma} P_{\rho}^{* \dagger}+$ H.c. & 302 & & 523 & \\
\hline$\varepsilon^{\mu \nu \lambda \rho} P_{\mu}^{*}\left\langle u_{\nu} h_{\lambda}{ }^{\sigma}\right\rangle u^{\delta} D_{\rho \sigma} P^{* \dagger}{ }_{\delta}+$ H.c. & & & 199 & & $\varepsilon^{\mu \nu \lambda \rho} P h_{\mu}{ }^{\sigma} f_{-\nu} \delta D_{\lambda \sigma \delta} P^{* \dagger} \rho+$ H.c. & 303 & & 524 & \\
\hline$\varepsilon^{\mu \nu \lambda \rho} P_{\mu}^{*}\left\langle u_{\nu} h^{\sigma \delta}\right\rangle u_{\lambda} D_{\rho \sigma} P^{* \dagger}{ }_{\delta}+$ H.c. & & & 200 & & $\varepsilon^{\mu \nu \lambda \rho} P h_{\mu}{ }^{\sigma} h_{\nu \sigma} D_{\lambda} P^{* \dagger} \rho+$ H.c. & 304 & & 525 & \\
\hline$\varepsilon^{\mu \nu \lambda \rho} P^{*}{ }_{\mu}\left\langle u^{\sigma} h_{\nu}{ }^{\delta}\right\rangle u_{\lambda} D_{\rho \delta} P^{* \dagger}{ }_{\sigma}+$ H.c. & & & 201 & & $\varepsilon^{\mu \nu \lambda \rho} P h_{\mu}{ }^{\sigma} h_{\nu}{ }^{\delta} D_{\lambda \sigma \delta} P^{* \dagger} \rho+$ H.c. & 305 & & 526 & \\
\hline$\varepsilon^{\mu \nu \lambda \rho} P_{\mu}^{*}\left\langle u_{\nu} u_{\lambda} f_{-\rho}{ }^{\sigma}\right\rangle P^{* \dagger}{ }_{\sigma}+$ H.c. & & & 202 & I & $\varepsilon^{\mu \nu \lambda \rho} P\left\langle u_{\mu} \nabla_{\nu} f_{-\lambda}^{\sigma}\right\rangle D_{\rho} P^{* \dagger}{ }_{\sigma}+$ H.c. & & & 527 & I \\
\hline$\varepsilon^{\mu \nu \lambda \rho} P^{*}{ }_{\mu}\left\langle u_{\nu} u_{\lambda} f_{-}{ }^{\sigma \delta}\right\rangle D_{\rho \sigma} P^{* \dagger}{ }_{\delta}+$ H.c. & & & 203 & I & $\varepsilon^{\mu \nu \lambda \rho} P\left\langle u_{\mu} \nabla_{\nu} f_{-\lambda}^{\sigma}\right\rangle D_{\sigma} P_{\rho \dagger}^{* \dagger}+$ H.c. & & & 528 & I \\
\hline$\varepsilon^{\mu \nu \lambda \rho} P^{*}{ }_{\mu}\left\langle u_{\nu} u_{\lambda} h_{\rho}{ }^{\sigma}\right\rangle P^{* \dagger}{ }_{\sigma}+$ H.c. & & & 204 & I & $\varepsilon^{\mu \nu \lambda \rho} P\left\langle u_{\mu} \nabla^{\sigma} f_{-\nu \sigma}\right\rangle D_{\lambda} P^{* \dagger}{ }_{\rho}+$ H.c. & & & 529 & I \\
\hline$\varepsilon^{\mu \nu \lambda \rho} P^{*}{ }_{\mu}\left\langle u_{\nu} u_{\lambda} h^{\sigma \delta}\right\rangle D_{\rho \sigma} P^{* \dagger}{ }_{\delta}+$ H.c. & & & 205 & I & $\varepsilon^{\mu \nu \lambda \rho} P\left\langle u_{\mu} \nabla^{\sigma} f_{-\nu}^{\delta}\right\rangle D_{\lambda \sigma \delta} P^{* \dagger} \rho+$ H.c. & & & 530 & I \\
\hline$\varepsilon^{\mu \nu \lambda \rho} P^{*}{ }_{\mu}\left\langle u_{\nu} u_{\lambda} h^{\sigma \delta}\right\rangle D_{\sigma \delta} P^{* \dagger} \rho$ & & & 206 & I & $\varepsilon^{\mu \nu \lambda \rho} P\left\langle u_{\mu} \nabla_{\nu} h^{\sigma \delta}\right\rangle D_{\lambda \sigma \delta} P_{\rho}^{* \dagger}+$ H.c. & & & 531 & I \\
\hline$P^{* \mu}\left\langle u_{\mu} \nabla^{\nu} f_{-\nu}^{\lambda}\right\rangle P_{\lambda}^{* \dagger}+$ H.c. & 93 & I & 207 & I & $\varepsilon^{\mu \nu \lambda \rho} P\left\langle f_{-\mu \nu} h_{\lambda}{ }^{\sigma}\right\rangle D_{\rho} P^{* \dagger}{ }_{\sigma}+$ H.c. & & & 532 & I \\
\hline$P^{* \mu}\left\langle u^{\nu} \nabla_{\mu} f_{-\nu}^{\lambda}\right\rangle P_{\lambda}^{* \dagger}+$ H.c. & 94 & I & 208 & I & $\varepsilon^{\mu \nu \lambda \rho} P\left\langle f_{-\mu \nu} h_{\lambda}{ }^{\sigma}\right\rangle D_{\sigma} P_{\rho}^{* \dagger}+$ H.c. & & & 533 & I \\
\hline$P^{* \mu}\left\langle u^{\nu} \nabla^{\lambda} f_{-\nu \lambda}\right\rangle P_{\mu}^{* \dagger}$ & 95 & & 209 & & $\varepsilon^{\mu \nu \lambda \rho} P\left\langle f_{-\mu \nu} h^{\sigma \delta}\right\rangle D_{\lambda \sigma \delta} P_{\rho}^{* \dagger}+$ H.c. & & & 534 & I \\
\hline$P^{* \mu}\left\langle u_{\mu} \nabla^{\nu} f_{-} \lambda \rho\right\rangle D_{\nu \lambda} P_{\rho}^{* \dagger}+$ H.c. & 96 & I & 210 & I & $P \nabla^{\mu} \nabla_{\mu} f_{-}{ }^{\nu \lambda} D_{\nu} P_{\lambda}^{* \dagger}+$ H.c. & 306 & I & 535 & I \\
\hline$P^{* \mu}\left\langle u^{\nu} \nabla_{\mu} f_{-}^{\lambda \rho}\right\rangle D_{\nu \lambda} P_{\rho}^{* \dagger}+$ H.c. & 97 & I & 211 & I & $P \nabla^{\mu} \nabla^{\nu} f_{-\mu}^{\lambda} D_{\nu} P^{* \dagger} \lambda+$ H.c. & 307 & I & 536 & I \\
\hline$P^{* \mu}\left\langle u^{\nu} \nabla^{\lambda} f_{-\nu}^{\rho}\right\rangle D_{\lambda \rho} P^{* \dagger} \mu$ & 98 & & 212 & & $P \nabla^{\mu} \nabla^{\nu} f_{-}^{\lambda \rho} D_{\mu \nu \lambda} P_{\rho}^{* \dagger}+$ H.c. & 308 & I & 537 & I \\
\hline$P^{* \mu}\left\langle u^{\nu} \nabla^{\lambda} f_{-\lambda}{ }^{\rho}\right\rangle D_{\nu \rho} P_{\mu}^{* \dagger}$ & 99 & & 213 & & $P \nabla^{\mu} \nabla^{\nu} h^{\lambda \rho} D_{\mu \nu \lambda} P_{\rho}^{* \dagger}+$ H.c. & 309 & I & 538 & I \\
\hline$P^{* \mu}\left\langle u^{\nu} \nabla_{\mu} h_{\nu}{ }^{\lambda}\right\rangle P^{* \dagger}{ }_{\lambda}^{*}+$ H.c. & 100 & I & 214 & I & $i \varepsilon^{\mu \nu \lambda \rho} P f_{+\mu \nu} u_{\lambda} u^{\sigma} D_{\rho} P^{* \dagger}{ }_{\sigma}+$ H.c. & 310 & & 539 & \\
\hline$P^{* \mu}\left\langle u_{\mu} \nabla^{\nu} h^{\lambda \rho}\right\rangle D_{\nu \lambda} P^{* \dagger} \rho+$ H.c. & 101 & I & 215 & I & $i \varepsilon^{\mu \nu \lambda \rho} P f_{+\mu \nu} u_{\lambda} u^{\sigma} D_{\sigma} P_{\rho}^{* \dagger}+$ H.c. & 311 & & 540 & \\
\hline$P^{* \mu}\left\langle u^{\nu} \nabla_{\mu} h^{\lambda \rho}\right\rangle D_{\nu \lambda} P_{\rho}^{* \dagger}+$ H.c. & 102 & I & 216 & $\mathrm{I}$ & $i \varepsilon^{\mu \nu \lambda \rho} P f_{+\mu \nu} u^{\sigma} u_{\lambda} D_{\rho} P^{* \dagger}{ }_{\sigma}+$ H.c. & 312 & & 541 & \\
\hline$P^{* \mu}\left\langle u^{\nu} \nabla_{\nu} h^{\lambda \rho}\right\rangle D_{\lambda \rho} P_{\mu}^{* \dagger}$ & 103 & & 217 & & $i \varepsilon^{\mu \nu \lambda \rho} P f_{+\mu \nu} u^{\sigma} u_{\lambda} D_{\sigma} P_{\rho}^{* \dagger}+$ H.c. & 313 & & 542 & \\
\hline$P^{* \mu}\left\langle u^{\nu} \nabla^{\lambda} h^{\rho \sigma}\right\rangle D_{\nu \lambda \rho \sigma} P_{\mu}^{* \dagger}$ & 104 & & 218 & & $i \varepsilon^{\mu \nu \lambda \rho} P f_{+\mu \nu} u^{\sigma} u_{\sigma} D_{\lambda} P_{\rho \dagger}^{* \dagger}+$ H.c. & 314 & & 543 & \\
\hline$P^{* \mu}\left\langle f_{-\mu}{ }^{\nu} h_{\nu}{ }^{\lambda}\right\rangle P^{* \dagger}{ }_{\lambda}+$ H.c. & 105 & I & 219 & I & $i \varepsilon^{\mu \nu \lambda \rho} P f_{+\mu \nu} u^{\sigma} u^{\delta} D_{\lambda \sigma \delta} P^{* \dagger}{ }_{\rho}+$ H.c. & 315 & & 544 & \\
\hline$P^{* \mu}\left\langle f_{-\mu}{ }^{\nu} h^{\lambda \rho}\right\rangle D_{\nu \lambda} P_{\rho \dagger}^{* \dagger}+$ H.c. & 106 & I & 220 & I & $i \varepsilon^{\mu \nu \lambda \rho} P u_{\mu} f_{+\nu \lambda} u^{\sigma} D_{\rho} P^{* \dagger}{ }_{\sigma}+$ H.c. & 316 & & 545 & \\
\hline$P^{* \mu}\left\langle f_{-}{ }^{\nu \lambda} h_{\nu}{ }^{\rho}\right\rangle D_{\lambda \rho} P_{\mu}^{* \dagger}$ & 107 & & 221 & & $i \varepsilon^{\mu \nu \lambda \rho} P u_{\mu} f_{+\nu \lambda} u^{\sigma} D_{\sigma} P_{\rho}^{* \dagger}+$ H.c. & 317 & & 546 & \\
\hline$P^{* \mu}\left\langle h_{\mu}{ }^{\nu} h_{\nu}{ }^{\lambda}\right\rangle P^{* \dagger} \lambda$ & 108 & I & 222 & I & $i \varepsilon^{\mu \nu \lambda \rho} P u_{\mu} f_{+\nu}{ }^{\sigma} u_{\lambda} D_{\rho} P^{* \dagger}{ }_{\sigma}+$ H.c. & 318 & & 547 & \\
\hline$P^{* \mu}\left\langle h^{\nu \lambda} h_{\nu \lambda}\right\rangle P_{\mu}^{* \dagger}$ & 109 & & 223 & & $i \varepsilon^{\mu \nu \lambda \rho} P u_{\mu} f_{+\nu}{ }^{\sigma} u_{\lambda} D_{\sigma} P^{* \dagger}{ }_{\rho}+$ H.c. & 319 & & 548 & \\
\hline$P^{* \mu}\left\langle h_{\mu}{ }^{\nu} h^{\lambda \rho}\right\rangle D_{\nu \lambda} P_{\rho}^{* \dagger}$ & 110 & I & 224 & I & $i \varepsilon^{\mu \nu \lambda \rho} P u_{\mu} f_{+\nu}{ }^{\sigma} u_{\sigma} D_{\lambda} P^{* \dagger}{ }_{\rho}+$ H.c. & 320 & & 549 & \\
\hline$P^{* \mu}\left\langle h_{\mu}{ }^{\nu} h^{\lambda \rho}\right\rangle D_{\lambda \rho} P_{\nu}^{* \dagger}$ & 111 & I & 225 & I & $i \varepsilon^{\mu \nu \lambda \rho} P u_{\mu} f_{+\nu}{ }^{\sigma} u^{\delta} D_{\lambda \sigma \delta} P_{\rho}^{* \dagger}+$ H.c. & 321 & & 550 & \\
\hline$P^{* \mu}\left\langle h^{\nu \lambda} h_{\nu}{ }^{\rho}\right\rangle D_{\lambda \rho} P_{\mu}^{* \dagger}$ & 112 & & 226 & & $i \varepsilon^{\mu \nu \lambda \rho} P u_{\mu} u_{\nu} f_{+\lambda}{ }^{\sigma} D_{\rho} P^{* \dagger}{ }_{\sigma}+$ H.c. & 322 & & 551 & \\
\hline$P^{* \mu}\left\langle h^{\nu \lambda} h^{\rho \sigma}\right\rangle D_{\nu \lambda \rho \sigma} P_{\mu}^{* \dagger}$ & 113 & & 227 & & $i \varepsilon^{\mu \nu \lambda \rho} P u_{\mu} u_{\nu} f_{+\lambda}^{\sigma} D_{\sigma} P^{* \dagger} \rho+$ H.c. & 323 & & 552 & \\
\hline$P^{* \mu} u_{\mu} \nabla^{\nu} f_{-\nu}^{\lambda} P^{* \dagger}{ }_{\lambda}+$ H.c. & 114 & & 228 & I & $i \varepsilon^{\mu \nu \lambda \rho} P\left\langle f_{+\mu \nu}\right\rangle u_{\lambda} u^{\sigma} D_{\rho} P^{* \dagger}{ }_{\sigma}+$ H.c. & 324 & & & \\
\hline$P^{* \mu} u^{\nu} \nabla_{\mu} f_{-\nu}^{\lambda} P_{\lambda}^{* \dagger}+$ H.c. & 115 & & 229 & I & $i \varepsilon^{\mu \nu \lambda \rho} P\left\langle f_{+\mu \nu}\right\rangle u_{\lambda} u^{\sigma} D_{\sigma} P_{\rho}^{* \dagger}+$ H.c. & 325 & & & \\
\hline$P^{* \mu} u^{\nu} \nabla_{\nu} f_{-\mu}^{\lambda} P_{\lambda}^{* \dagger}+$ H.c. & & & 230 & I & $i \varepsilon^{\mu \nu \lambda \rho} P\left\langle f_{+\mu \nu}\right\rangle u^{\sigma} u_{\sigma} D_{\lambda} P^{* \dagger}{ }_{\rho}+$ H.c. & 326 & & & \\
\hline$P^{* \mu} u^{\nu} \nabla^{\lambda} f_{-\mu \lambda} P^{* \dagger}{ }_{\nu}+$ H.c. & & & 231 & & $i \varepsilon^{\mu \nu \lambda \rho} P\left\langle f_{+\mu \nu}\right\rangle u^{\sigma} u^{\delta} D_{\lambda \sigma \delta} P_{\rho}^{* \dagger}+$ H.c. & 327 & & & \\
\hline$P^{* \mu} u^{\nu} \nabla^{\lambda} f_{-\nu \lambda} P_{\mu}^{* \dagger}+$ H.c. & & & 232 & & $i \varepsilon^{\mu \nu \lambda \rho} P u_{\mu} u^{\sigma} f_{+\nu \lambda} D_{\rho} P^{* \dagger}{ }_{\sigma}+$ H.c. & & & 553 & \\
\hline$P^{* \mu} u_{\mu} \nabla^{\nu} f_{-}^{\lambda \rho} D_{\nu \lambda} P_{\rho}^{* \dagger}+$ H.c. & 116 & & 233 & I & $i \varepsilon^{\mu \nu \lambda \rho} P u_{\mu} u^{\sigma} f_{+\nu \lambda} D_{\sigma} P_{\rho}^{* \dagger}+$ H.c. & & & 554 & \\
\hline
\end{tabular}




\begin{tabular}{|c|c|c|c|c|c|c|c|}
\hline$O_{n}$ & $S U(2)$ & $\tilde{c}_{n}^{(4)}$ & $S U(3)$ & $\tilde{C}_{n}^{(4)}$ & $O_{n}$ & $S U(2) \tilde{c}_{n}^{(4)}$ & $S U(3) \tilde{C}_{n}^{(4)}$ \\
\hline$P^{* \mu} u^{\nu} \nabla_{\mu} f_{-}^{\lambda \rho} D_{\nu \lambda} P^{* \dagger}{ }_{\rho}+$ H.c. & 117 & & 234 & I & $i \varepsilon^{\mu \nu \lambda \rho} P u_{\mu} u^{\sigma} f_{+\nu \sigma} D_{\lambda} P^{* \dagger}{ }_{\rho}+$ H.c. & & 555 \\
\hline$P^{* \mu} u^{\nu} \nabla^{\lambda} f_{-\mu}{ }^{\rho} D_{\nu \lambda} P^{* \dagger}{ }_{\rho}^{\rho}+$ H.c. & & & 235 & I & $i \varepsilon^{\mu \nu \lambda \rho} P u_{\mu} u^{\sigma} f_{+\nu}^{\delta} D_{\lambda \sigma \delta} P^{* \dagger} \rho+$ H.c. & & 556 \\
\hline$P^{* \mu} u^{\nu} \nabla^{\lambda} f_{-\mu}{ }^{\rho} D_{\lambda \rho} P^{* \dagger}{ }_{\nu}+$ H.c. & & & 236 & & $i \varepsilon^{\mu \nu \lambda \rho} P\left\langle f_{+\mu \nu} u_{\lambda}\right\rangle u^{\sigma} D_{\rho} P^{* \dagger}{ }_{\sigma}+$ H.c. & & 557 \\
\hline$P^{* \mu} u^{\nu} \nabla^{\lambda} f_{-\nu}{ }^{\rho} D_{\lambda \rho} P^{* \dagger}{ }_{\mu}+$ H.c. & & & 237 & & $i \varepsilon^{\mu \nu \lambda \rho} P\left\langle f_{+\mu \nu} u_{\lambda}\right\rangle u^{\sigma} D_{\sigma} P_{\rho}^{* \dagger}+$ H.c. & & 558 \\
\hline$P^{* \mu} u^{\nu} \nabla^{\lambda} f_{-\lambda}{ }^{\rho} D_{\nu \rho} P^{* \dagger}{ }_{\mu}+$ H.c. & & & 238 & & $i \varepsilon^{\mu \nu \lambda \rho} P\left\langle f_{+\mu \nu} u^{\sigma}\right\rangle u_{\lambda} D_{\rho} P^{* \dagger}{ }_{\sigma}+$ H.c. & & 559 \\
\hline$P^{* \mu} u^{\nu} \nabla_{\mu} h_{\nu}^{\lambda} P_{\lambda}^{* \dagger}+$ H.c. & & & 239 & I & $i \varepsilon^{\mu \nu \lambda \rho} P\left\langle f_{+\mu \nu} u^{\sigma}\right\rangle u_{\lambda} D_{\sigma} P_{\rho}^{* \dagger}+$ H.c. & & 560 \\
\hline$P^{* \mu} u_{\mu} \nabla^{\nu} h^{\lambda \rho} D_{\nu \lambda} P^{* \dagger} \rho+$ H.c. & 118 & & 240 & & $i \varepsilon^{\mu \nu \lambda \rho} P\left\langle f_{+\mu \nu} u^{\sigma}\right\rangle u_{\sigma} D_{\lambda} P_{\rho}^{* \dagger}+$ H.c. & & 561 \\
\hline$P^{* \mu} u^{\nu} \nabla_{\mu} h^{\lambda \rho} D_{\nu \lambda} P^{* \dagger} \rho+$ H.c. & & & 241 & I & $i \varepsilon^{\mu \nu \lambda \rho} P\left\langle f_{+\mu \nu} u^{\sigma}\right\rangle u^{\delta} D_{\lambda \sigma \delta} P^{* \dagger}{ }_{\rho}+$ H.c. & & 562 \\
\hline$P^{* \mu} u^{\nu} \nabla_{\mu} h^{\lambda \rho} D_{\lambda \rho} P^{* \dagger}{ }_{\nu}+$ H.c. & & & 242 & & $i \varepsilon^{\mu \nu \lambda \rho} P\left\langle f_{+\mu \nu} u_{\lambda} u^{\sigma}\right\rangle D_{\rho} P^{* \dagger}{ }_{\sigma}+$ H.c. & & 563 \\
\hline$P^{* \mu} u^{\nu} \nabla_{\nu} h^{\lambda \rho} D_{\lambda \rho} P^{* \dagger}{ }_{\mu}+$ H.c. & & & 243 & & $i \varepsilon^{\mu \nu \lambda \rho} P\left\langle f_{+\mu \nu} u_{\lambda} u^{\sigma}\right\rangle D_{\sigma} P^{* \dagger}{ }_{\rho}+$ H.c. & & 564 \\
\hline$P^{* \mu} u^{\nu} \nabla^{\lambda} h^{\rho \sigma} D_{\nu \lambda \rho \sigma} P^{* \dagger}{ }_{\mu}+$ H.c. & & & 244 & & $i \varepsilon^{\mu \nu \lambda \rho} P\left\langle f_{+\mu \nu} u^{\sigma} u_{\lambda}\right\rangle D_{\rho} P^{* \dagger}{ }_{\sigma}+$ H.c. & & 565 \\
\hline$P^{* \mu} f_{-\mu}^{\nu} f_{-\nu}{ }^{\lambda} P^{* \dagger}{ }_{\lambda}$ & 119 & I & 245 & I & $i \varepsilon^{\mu \nu \lambda \rho} P\left\langle f_{+\mu \nu} u^{\sigma} u_{\lambda}\right\rangle D_{\sigma} P^{* \dagger}{ }_{\rho}+$ H.c. & & 566 \\
\hline$P^{* \mu} f_{-}{ }^{\nu \lambda} f_{-\mu \nu} P^{* \dagger} \lambda$ & & & 246 & & $i \varepsilon^{\mu \nu \lambda \rho} P\left\langle f_{+\mu \nu} u^{\sigma} u_{\sigma}\right\rangle D_{\lambda} P^{* \dagger}{ }_{\rho}+$ H.c. & & 567 \\
\hline$P^{* \mu} f_{-}{ }^{\nu \lambda} f_{-\nu \lambda} P^{* \dagger}{ }_{\mu}$ & & & 247 & & $i \varepsilon^{\mu \nu \lambda \rho} P\left\langle f_{+\mu \nu} u^{\sigma} u^{\delta}\right\rangle D_{\lambda \sigma \delta} P^{* \dagger}{ }_{\rho}+$ H.c. & & 568 \\
\hline$P^{* \mu} f_{-\mu}^{\nu} f_{-}^{\lambda \rho} D_{\nu \lambda} P^{* \dagger} \rho$ & 120 & I & 248 & I & $i P f_{+}{ }^{\mu \nu} f_{-\mu}^{\lambda} D_{\nu} P^{* \dagger}{ }_{\lambda}+$ H.c. & 328 & 569 \\
\hline$P^{* \mu} f_{-}^{\nu \lambda} f_{-\mu}^{\rho} D_{\nu \rho} P^{* \dagger} \lambda$ & & & 249 & & $i P f_{+}{ }^{\mu \nu} f_{-\mu}^{\lambda} D_{\lambda} P^{* \dagger}{ }_{\nu}+$ H.c. & 329 & 570 \\
\hline$P^{* \mu} f_{-}{ }^{\nu \lambda} f_{-\nu}^{\mu}{ }^{\rho} D_{\lambda \rho} P^{* \dagger}{ }_{\mu}$ & & & 250 & & $i P f_{-}^{\mu \nu} f_{+\mu}^{\lambda} D_{\nu} P^{* \dagger} \lambda+$ H.c. & 330 & 571 \\
\hline$P^{* \mu} f_{-\mu}{ }^{\nu} h_{\nu}{ }^{\lambda} P^{* \dagger}{ }_{\lambda}+$ H.c. & 121 & & 251 & I & $i P f_{-}{ }^{\mu \nu} f_{+\mu}{ }^{\lambda} D_{\lambda} P^{* \dagger}{ }_{\nu}+$ H.c. & 331 & 572 \\
\hline$P^{* \mu} f_{-}{ }^{\nu \lambda} h_{\mu \nu} P_{\lambda}^{* \dagger}{ }_{\lambda}+$ H.c. & & & 252 & & $i P f_{+}{ }^{\mu \nu} h_{\mu}{ }^{\lambda} D_{\nu} P^{* \dagger}{ }_{\lambda}+$ H.c. & 332 & 573 \\
\hline$P^{* \mu} f_{-\mu}{ }^{\nu} h^{\lambda \rho} D_{\nu \lambda} P^{* \dagger} \rho+$ H.c. & 122 & & 253 & I & $i P f_{+}{ }^{\mu \nu} h_{\mu}{ }^{\lambda} D_{\lambda} P^{* \dagger}{ }_{\nu}+$ H.c. & 333 & 574 \\
\hline$P^{* \mu} f_{-\mu}{ }^{\nu} h^{\lambda \rho} D_{\lambda \rho} P^{* \dagger}{ }_{\nu}+$ H.c. & 123 & I & 254 & I & $i P h^{\mu \nu} f_{+\mu}^{\lambda} D_{\nu} P^{* \dagger}{ }_{\lambda}+$ H.c. & 334 & 575 \\
\hline$P^{* \mu} f_{-}{ }^{\nu \lambda} h_{\mu}{ }^{\rho} D_{\nu \rho} P^{* \dagger}{ }_{\lambda}+$ H.c. & & & 255 & & $i P h^{\mu \nu} f_{+\mu}{ }^{\lambda} D_{\lambda} P^{* \dagger}{ }_{\nu}+$ H.c. & 335 & 576 \\
\hline$P^{* \mu} f_{-}{ }^{\nu \lambda} h_{\nu}{ }^{\rho} D_{\lambda \rho} P^{* \dagger}{ }_{\mu}+$ H.c. & & & 256 & & $i P f_{+}{ }^{\mu \nu} h^{\lambda \rho} D_{\mu \lambda \rho} P^{* \dagger}{ }_{\nu}+$ H.c. & 336 & 577 \\
\hline$P^{* \mu} h_{\mu}{ }^{\nu} h_{\nu}{ }^{\lambda} P^{* \dagger}{ }_{\lambda}$ & 124 & & 257 & I & $i P h^{\mu \nu} f_{+}{ }^{\lambda \rho} D_{\mu \nu \lambda} P^{* \dagger}{ }_{\rho}+$ H.c. & 337 & 578 \\
\hline$P^{* \mu} h^{\nu \lambda} h_{\mu \nu} P_{\lambda}^{* \dagger}$ & & & 258 & & $i P \nabla^{\mu} f_{+\mu}{ }^{\nu} u^{\lambda} D_{\nu} P^{* \dagger}{ }_{\lambda}+$ H.c. & 338 & 579 \\
\hline$P^{* \mu} h^{\nu \lambda} h_{\nu \lambda} P^{* \dagger}{ }_{\mu}$ & & & 259 & & $i P \nabla^{\mu} f_{+\mu}{ }^{\nu} u^{\lambda} D_{\lambda} P^{* \dagger}{ }_{\nu}+$ H.c. & 339 & 580 \\
\hline$P^{* \mu} h_{\mu}{ }^{\nu} h^{\lambda \rho} D_{\nu \lambda} P^{* \dagger} \rho$ & 125 & & 260 & I & $i P \nabla^{\mu} f_{+}{ }^{\nu \lambda} u_{\mu} D_{\nu} P^{* \dagger}{ }_{\lambda}+$ H.c. & 340 & 581 \\
\hline$P^{* \mu} h_{\mu}{ }^{\nu} h^{\lambda \rho} D_{\lambda \rho} P_{\nu}^{* \dagger}{ }_{\nu}+$ H.c. & & & 261 & I & $i P \nabla^{\mu} f_{+}{ }^{\nu \lambda} u_{\nu} D_{\mu} P^{* \dagger} \lambda+$ H.c. & 341 & 582 \\
\hline$P^{* \mu} h^{\nu \lambda} h_{\mu}{ }^{\rho} D_{\nu \rho} P_{\lambda}^{* \dagger}$ & & & 262 & & $i P u^{\mu} \nabla_{\mu} f_{+}{ }^{\nu \lambda} D_{\nu} P^{* \dagger} \lambda+$ H.c. & 342 & 583 \\
\hline$P^{* \mu} h^{\nu \lambda} h_{\nu}{ }^{\rho} D_{\lambda \rho} P^{* \dagger}{ }_{\mu}{ }^{\lambda}$ & & & 263 & & $i P u^{\mu} \nabla^{\nu} f_{+\mu}^{\lambda} D_{\nu} P^{* \dagger} \lambda+$ H.c. & 343 & 584 \\
\hline$P^{* \mu} h^{\nu \lambda} h^{\rho \sigma} D_{\nu \lambda \rho \sigma}^{\mu} P^{* \dagger}{ }_{\mu}$ & & & 264 & & $i P u^{\mu} \nabla^{\nu} f_{+\nu}^{\lambda} D_{\mu} P^{* \dagger} \lambda+$ H.c. & 344 & 585 \\
\hline$\varepsilon^{\mu \nu \lambda \rho} P^{*}{ }_{\mu} \nabla_{\nu} \nabla^{\sigma} f_{-\lambda}{ }^{\delta} D_{\rho \sigma} P^{* \dagger}{ }_{\delta}+$ H.c. & 126 & I & 265 & I & $i P u^{\mu} \nabla^{\nu} f_{+\nu}{ }^{\lambda} D_{\lambda} P^{* \dagger}{ }_{\mu}+$ H.c. & 345 & 586 \\
\hline$\varepsilon^{\mu \nu \lambda \rho} P^{*}{ }_{\mu}^{\mu} \nabla^{\sigma} \nabla^{\delta} f_{-\nu \lambda} D_{\rho \sigma} P^{* \dagger}{ }_{\delta}+$ H.c. & 127 & I & 266 & I & $i P \nabla^{\mu} f_{+}{ }^{\nu \lambda} u^{\rho} D_{\mu \nu \rho} P^{* \dagger}{ }_{\lambda}+$ H.c. & 346 & 587 \\
\hline$i P^{* \mu}\left\langle f_{+\mu}{ }^{\mu}\right\rangle u_{\nu} u^{\lambda} P^{* \dagger}{ }_{\lambda}+$ H.c. & 128 & I & & & $i P u^{\mu} \nabla^{\nu} f_{+}{ }^{\lambda \rho} D_{\mu \nu \lambda} P_{\rho}^{* \dagger}+$ H.c. & 347 & 588 \\
\hline$i P^{* \mu}\left\langle f_{+\mu}{ }^{\nu}\right\rangle u^{\lambda} u_{\nu} P_{\lambda}^{* \dagger}{ }_{\lambda}+$ H.c. & 129 & & & & $i P\left\langle f_{+}{ }^{\mu \nu}\right\rangle f_{-\mu}{ }^{\lambda} D_{\nu} P^{* \dagger}{ }_{\lambda}+$ H.c. & 348 & \\
\hline$i P^{* \mu}\left\langle f_{+\mu}{ }^{\nu}\right\rangle u^{\lambda} u_{\lambda} P_{\nu}^{* \dagger}$ & 130 & I & & & $i P\left\langle f_{+}{ }^{\mu \nu}\right\rangle h_{\mu}{ }^{\lambda} D_{\nu} P^{* \dagger} \lambda+$ H.c. & 349 & \\
\hline$i P^{* \mu}\left\langle f_{+}{ }^{\nu \lambda}\right\rangle u_{\nu} u_{\lambda} P^{* \dagger}{ }_{\mu}$ & 131 & & & & $i P\left\langle f_{+}{ }^{\mu \nu}\right\rangle h_{\mu}{ }^{\lambda} D_{\lambda} P^{* \dagger}{ }_{\nu}+$ H.c. & 350 & \\
\hline$i P^{* \mu}\left\langle f_{+\mu}{ }^{\nu}\right\rangle u^{\lambda} u^{\rho} D_{\nu \lambda} P^{* \dagger} \rho+$ H.c. & 132 & I & & & $i P\left\langle f_{+}{ }^{\mu \nu}\right\rangle h^{\lambda \rho} D_{\mu \lambda \rho} P^{* \dagger}{ }_{\nu}+$ H.c. & 351 & \\
\hline$i P^{* \mu}\left\langle f_{+\mu}{ }^{\nu}\right\rangle u^{\lambda} u^{\rho} D_{\nu \rho} P^{* \dagger}{ }_{\lambda}+$ H.c. & 133 & & & & $i P\left\langle\nabla^{\mu} f_{+\mu}{ }^{\nu}\right\rangle u^{\lambda} D_{\nu} P^{* \dagger}{ }_{\lambda}+$ H.c. & 352 & \\
\hline$i P^{* \mu}\left\langle f_{+\mu}{ }^{\nu}\right\rangle u^{\lambda} u^{\rho} D_{\lambda \rho} P^{* \dagger}{ }_{\nu}$ & 134 & I & & & $i P\left\langle\nabla^{\mu} f_{+\mu}{ }^{\nu}\right\rangle u^{\lambda} D_{\lambda} P^{* \dagger}{ }_{\nu}+$ H.c. & 353 & \\
\hline$i P^{* \mu}\left\langle f_{+}{ }^{\nu \lambda}\right\rangle u_{\nu} u^{\rho} D_{\lambda \rho} P^{* \dagger}{ }_{\mu}+$ H.c. & 135 & & & & $i P\left\langle\nabla^{\mu} f_{+}{ }^{\nu \lambda}\right\rangle u_{\mu} D_{\nu} P_{\lambda}^{* \dagger}+$ H.c. & 354 & \\
\hline$i P^{* \mu}\left\langle f_{+\mu}^{\nu} u_{\nu}\right\rangle u^{\lambda} P_{\lambda}^{* \dagger}+$ H.c. & 136 & I & 267 & I & $i P\left\langle\nabla^{\mu} f_{+}{ }^{\nu \lambda}\right\rangle u_{\nu} D_{\mu} P_{\lambda}^{* \dagger}+$ H.c. & 355 & \\
\hline$i P^{* \mu}\left\langle f_{+\mu}{ }^{\nu} u^{\lambda}\right\rangle u_{\nu} P_{\lambda}^{* \dagger}+$ H.c. & 137 & I & 268 & I & $i P\left\langle\nabla^{\mu} f_{+}{ }^{\nu \lambda}\right\rangle u^{\rho} D_{\mu \nu \rho} P_{\lambda}^{* \dagger}{ }_{\lambda}+$ H.c. & 356 & \\
\hline$i P^{* \mu}\left\langle f_{+\mu}{ }^{\nu} u^{\lambda}\right\rangle u_{\lambda} P_{\nu}^{* \dagger}$ & 138 & I & 269 & I & $i P\left\langle f_{+}{ }^{\mu \nu} f_{-\mu}{ }^{\lambda}\right\rangle D_{\nu} P^{* \dagger}{ }_{\lambda}+$ H.c. & & 589 \\
\hline$i P^{* \mu}\left\langle f_{+\mu}{ }^{\nu} u^{\lambda}\right\rangle u^{\rho} D_{\nu \lambda} P^{* \dagger} \rho+$ H.c. & 139 & I & 270 & I & $i P\left\langle f_{+}{ }^{\mu \nu} h_{\mu}{ }^{\lambda}\right\rangle D_{\nu} P^{* \dagger}{ }_{\lambda}+$ H.c. & & 590 \\
\hline$i P^{* \mu}\left\langle f_{+\mu}{ }^{\nu} u^{\lambda}\right\rangle u^{\rho} D_{\nu \rho} P^{* \dagger}{ }_{\lambda}+$ H.c. & 140 & I & 271 & I & $i P\left\langle f_{+}{ }^{\mu \nu} h_{\mu}{ }^{\lambda}\right\rangle D_{\lambda} P^{* \dagger}{ }_{\nu}+$ H.c. & & 591 \\
\hline$i P^{* \mu}\left\langle f_{+\mu}{ }^{\nu} u^{\lambda}\right\rangle u^{\rho} D_{\lambda \rho} P_{\nu}^{* \dagger}$ & 141 & I & 272 & I & $i P\left\langle f_{+}{ }^{\mu \nu} h^{\lambda \rho}\right\rangle D_{\mu \lambda \rho} P^{* \dagger}{ }_{\nu}+$ H.c. & & 592 \\
\hline$i P^{* \mu}\left\langle f_{+\mu}{ }^{\nu} u_{\nu} u^{\lambda}\right\rangle P_{\lambda}^{* \dagger}{ }_{\lambda}+$ H.c. & 142 & I & 273 & I & $i P\left\langle\nabla^{\mu} f_{+\mu}{ }^{\nu} u^{\lambda}\right\rangle D_{\nu} P^{* \dagger}{ }_{\lambda}+$ H.c. & & 593 \\
\hline
\end{tabular}




\begin{tabular}{|c|c|c|c|c|c|c|c|c|c|}
\hline$O_{n}$ & $S U(2)$ & $\tilde{c}_{n}^{(4)}$ & $S U(3)$ & $\tilde{C}_{n}^{(4)}$ & $O_{n}$ & $S U(2)$ & $\tilde{c}_{n}^{(4)}$ & $S U(3)$ & $\tilde{C}_{n}^{(4)}$ \\
\hline$i P^{* \mu}\left\langle f_{+\mu}{ }^{\nu} u^{\lambda} u_{\nu}\right\rangle P^{* \dagger} \lambda+$ H.c. & & & 274 & & $i P\left\langle\nabla^{\mu} f_{+\mu}{ }^{\nu} u^{\lambda}\right\rangle D_{\lambda} P^{* \dagger}{ }_{\nu}+$ H.c. & & & 594 & \\
\hline$i P^{* \mu}\left\langle f_{+\mu}{ }^{\nu} u^{\lambda} u_{\lambda}\right\rangle P^{* \dagger}{ }_{\nu}$ & & & 275 & I & $i P\left\langle\nabla^{\mu} f_{+}{ }^{\nu \lambda} u_{\mu}\right\rangle D_{\nu} P^{* \dagger}{ }_{\lambda}+$ H.c. & & & 595 & \\
\hline$i P^{* \mu}\left\langle f_{+}{ }^{\nu \lambda} u_{\nu} u_{\lambda}\right\rangle P^{* \dagger}{ }_{\mu}$ & 143 & & 276 & & $i P\left\langle\nabla^{\mu} f_{+}{ }^{\nu \lambda} u_{\nu}\right\rangle D_{\mu} P^{* \dagger} \lambda+$ H.c. & & & 596 & \\
\hline$i P^{* \mu}\left\langle f_{+\mu}{ }^{\nu} u^{\lambda} u^{\rho}\right\rangle D_{\nu \lambda} P_{\rho}^{* \dagger}+$ H.c. & 144 & I & 277 & I & $i P\left\langle\nabla^{\mu} f_{+}{ }^{\nu \lambda} u^{\rho}\right\rangle D_{\mu \nu \rho} P_{\lambda}^{* \dagger}+$ H.c. & & & 597 & \\
\hline$i P^{* \mu}\left\langle f_{+\mu}{ }^{\nu} u^{\lambda} u^{\rho}\right\rangle D_{\nu \rho} P^{* \dagger}{ }_{\lambda}+$ H.c. & & & 278 & & $i \varepsilon^{\mu \nu \lambda \rho} P \nabla_{\mu} \nabla^{\sigma} f_{+\nu \sigma} D_{\lambda} P^{* \dagger}{ }_{\rho}+$ H.c. & 357 & & 598 & \\
\hline$i P^{* \mu}\left\langle f_{+\mu}{ }^{\nu} u^{\lambda} u^{\rho}\right\rangle D_{\lambda \rho} P^{* \dagger}{ }_{\nu}$ & & & 279 & I & $i \varepsilon^{\mu \nu \lambda \rho} P \nabla_{\mu}^{\mu} \nabla^{\sigma} f_{+\nu}{ }^{\delta} D_{\lambda \sigma \delta} P_{\rho}^{* \dagger}+$ H.c. & 358 & & 599 & \\
\hline$i P^{* \mu}\left\langle f_{+}{ }^{\nu \lambda} u_{\nu} u^{\rho}\right\rangle D_{\lambda \rho} P_{\mu}^{* \dagger}+$ H.c. & 145 & & 280 & & $\varepsilon^{\mu \nu \lambda \rho} P f_{+\mu \nu}^{\mu} f_{+\lambda}{ }^{\sigma} D_{\rho} P_{\sigma}^{* \dagger}+$ H.c. & 359 & & 600 & \\
\hline$i P^{* \mu}\left\langle u_{\mu} u^{\nu}\right\rangle f_{+\nu}^{\lambda} P_{\lambda}^{* \dagger}+$ H.c. & 146 & I & 281 & I & $\varepsilon^{\mu \nu \lambda \rho} P f_{+\mu \nu} f_{+\lambda}{ }^{\sigma} D_{\sigma} P_{\rho}^{* \dagger}+$ H.c. & 360 & & 601 & \\
\hline$i P^{* \mu}\left\langle u^{\nu} u_{\nu}\right\rangle f_{+\mu}^{\lambda} P^{* \dagger} \lambda$ & 147 & I & 282 & I & $\varepsilon^{\mu \nu \lambda \rho} P\left\langle f_{+\mu \nu}\right\rangle f_{+\lambda}{ }^{\sigma} D_{\rho} P^{* \dagger}{ }_{\sigma}+$ H.c. & 361 & I & & \\
\hline$i P^{* \mu}\left\langle u_{\mu} u^{\nu}\right\rangle f_{+}^{\lambda \rho} D_{\nu \lambda} P_{\rho}^{* \dagger}+$ H.c. & 148 & I & 283 & I & $\varepsilon^{\mu \nu \lambda \rho} P\left\langle f_{+\mu \nu}\right\rangle f_{+\lambda}{ }^{\sigma} D_{\sigma} P^{* \dagger} \rho+$ H.c. & 362 & I & & \\
\hline$i P^{* \mu}\left\langle u^{\nu} u^{\lambda}\right\rangle f_{+\mu}^{\rho} D_{\nu \lambda} P^{* \dagger} \rho$ & 149 & I & 284 & I & $\varepsilon^{\mu \nu \lambda \rho} P u_{\mu} u_{\nu} \chi_{+} D_{\lambda} P_{\rho}^{* \dagger}+$ H.c. & 363 & & 602 & \\
\hline$i P^{* \mu} f_{+\mu}{ }^{\nu} u_{\nu} u^{\lambda} P_{\lambda}^{* \dagger}+$ H.c. & & & 285 & I & $\varepsilon^{\mu \nu \lambda \rho} P u_{\mu} \chi_{+} u_{\nu} D_{\lambda} P_{\rho}^{* \dagger}+$ H.c. & 364 & & 603 & \\
\hline$i P^{* \mu} f_{+\mu}{ }^{\nu} u^{\lambda} u_{\nu} P_{\lambda}^{* \dagger}{ }_{\lambda}+$ H.c. & & & 286 & I & $\varepsilon^{\mu \nu \lambda \rho} P \chi_{+} u_{\mu} u_{\nu} D_{\lambda} P_{\rho}^{* \dagger}+$ H.c. & 365 & & 604 & \\
\hline$i P^{* \mu} f_{+\mu}{ }^{\nu} u^{\lambda} u_{\lambda} P^{* \dagger}{ }_{\nu}+$ H.c. & & & 287 & I & $\varepsilon^{\mu \nu \lambda \rho} P\left\langle u_{\mu} u_{\nu} \chi_{+}\right\rangle D_{\lambda} P_{\rho}^{* \dagger}+$ H.c. & & & 605 & \\
\hline$i P^{* \mu} f_{+}{ }^{\nu \lambda} u_{\mu} u_{\nu} P_{\lambda}^{* \dagger}+$ H.c. & & & 288 & & $\varepsilon^{\mu \nu \lambda \rho} P\left\langle u_{\mu} \chi_{+}\right\rangle u_{\nu} D_{\lambda} P_{\rho}^{* \dagger}+$ H.c. & & & 606 & I \\
\hline$i P^{* \mu} f_{+}{ }^{\nu \lambda} u_{\nu} u_{\mu} P^{* \dagger} \lambda+$ H.c. & & & 289 & & $\varepsilon^{\mu \nu \lambda \rho} P\left\langle\chi_{+}\right\rangle u_{\mu} u_{\nu} D_{\lambda} P^{* \dagger} \rho+$ H.c. & & & 607 & \\
\hline$i P^{* \mu} f_{+}{ }^{\nu \lambda} u_{\nu} u_{\lambda} P^{* \dagger}{ }_{\mu}+$ H.c. & & & 290 & & $P f_{-}{ }^{\mu \nu} \chi_{+} D_{\mu} P^{* \dagger}{ }_{\nu}+$ H.c. & 366 & & 608 & \\
\hline$i P^{* \mu} f_{+\mu}{ }^{\nu} u^{\lambda} u^{\rho} D_{\nu \lambda} P^{* \dagger} \rho+$ H.c. & & & 291 & I & $P \chi_{+} f_{-}{ }^{\mu \nu} D_{\mu} P^{* \dagger}{ }_{\nu}+$ H.c. & 367 & & 609 & \\
\hline$i P^{* \mu} f_{+\mu}{ }^{\nu} u^{\lambda} u^{\rho} D_{\nu \rho} P^{* \dagger} \lambda+$ H.c. & & & 292 & I & $P h^{\mu \nu} \chi_{+} D_{\mu} P_{\nu}^{* \dagger}+$ H.c. & 368 & & 610 & \\
\hline$i P^{* \mu} f_{+\mu}{ }^{\nu} u^{\lambda} u^{\rho} D_{\lambda \rho} P^{* \dagger}{ }_{\nu}+$ H.c. & & & 293 & I & $P \chi_{+} h^{\mu \nu} D_{\mu} P_{\nu \dagger}^{* \dagger}+$ H.c. & 369 & & 611 & \\
\hline$i P^{* \mu} f_{+}{ }^{\nu \lambda} u_{\mu} u^{\rho} D_{\nu \rho} P^{* \dagger}{ }_{\lambda}+$ H.c. & & & 294 & & $P u^{\mu} \nabla^{\nu} \chi_{+} D_{\mu} P_{\nu}^{* \dagger}{ }_{\nu}+$ H.c. & 370 & & 612 & \\
\hline$i P^{* \mu} f_{+}{ }^{\nu \lambda} u_{\nu} u^{\rho} D_{\lambda \rho} P^{* \dagger}{ }_{\mu}+$ H.c. & & & 295 & & $P u^{\mu} \nabla^{\nu} \chi_{+} D_{\nu} P^{* \dagger}{ }_{\mu}+$ H.c. & 371 & & 613 & \\
\hline$i P^{* \mu} f_{+}{ }^{\nu \lambda} u^{\rho} u_{\mu} D_{\nu \rho} P^{* \dagger}{ }_{\lambda}+$ H.c. & & & 296 & & $P \nabla^{\mu} \chi_{+} u^{\nu} D_{\mu} P^{* \dagger}{ }_{\nu}+$ H.c. & 372 & & 614 & \\
\hline$i P^{* \mu} f_{+}{ }^{\nu \lambda} u^{\rho} u_{\nu} D_{\lambda \rho} P^{* \dagger}{ }_{\mu}+$ H.c. & & & 297 & & $P \nabla^{\mu} \chi_{+} u^{\nu} D_{\nu} P_{\mu}^{* \dagger}+$ H.c. & 373 & & 615 & \\
\hline$i P^{* \mu} u_{\mu} f_{+}{ }^{\nu \lambda} u_{\nu} P^{* \dagger}{ }_{\lambda}+$ H.c. & & & 298 & I & $P\left\langle f_{-}^{\mu \nu} \chi_{+}\right\rangle D_{\mu} P^{* \dagger}{ }_{\nu}+$ H.c. & 374 & I & 616 & I \\
\hline$i P^{* \mu} u^{\nu} f_{+\nu}^{\lambda} u_{\lambda} P^{* \dagger} \mu$ & & & 299 & & $P\left\langle\chi_{+}\right\rangle f_{-}^{\mu \nu} D_{\mu} P^{* \dagger}{ }_{\nu}+$ H.c. & & & 617 & I \\
\hline$i P^{* \mu} u_{\mu} f_{+}{ }^{\nu \lambda} u^{\rho} D_{\nu \rho} P_{\lambda}^{* \dagger}+$ H.c. & & & 300 & & $P\left\langle h^{\mu \nu} \chi_{+}\right\rangle D_{\mu} P_{\nu \dagger}^{* \dagger}+$ H.c. & 375 & I & 618 & I \\
\hline$i P^{* \mu} u^{\nu} f_{+\nu}{ }^{\lambda} u^{\rho} D_{\lambda \rho} P^{* \dagger}{ }_{\mu}+$ H.c. & & & 301 & & $P\left\langle\chi_{+}\right\rangle h^{\mu \nu} D_{\mu} P_{\nu}^{* \dagger}+$ H.c. & & & 619 & I \\
\hline$i \varepsilon^{\mu \nu \lambda \rho} P^{*}{ }_{\mu} \nabla_{\nu} f_{+\lambda}{ }^{\sigma} u_{\rho} P^{* \dagger}{ }_{\sigma}+$ H.c. & 150 & & 302 & & $P\left\langle u^{\mu} \nabla^{\nu} \chi_{+}\right\rangle D_{\mu} P^{* \dagger}{ }_{\nu}+$ H.c. & 376 & I & 620 & I \\
\hline$i \varepsilon^{\mu \nu \lambda \rho} P_{\mu}^{*} \nabla_{\nu} f_{+\lambda}{ }^{\sigma} u_{\sigma} P_{\rho}^{* \dagger}+$ H.c. & 151 & I & 303 & I & $P\left\langle\nabla^{\mu} \chi_{+}\right\rangle u^{\nu} D_{\nu} P^{* \dagger}{ }_{\mu}+$ H.c. & & & 621 & I \\
\hline$i \varepsilon^{\mu \nu \lambda \rho} P_{\mu}^{*} \nabla^{\sigma} f_{+\nu \sigma} u_{\lambda} P_{\rho}^{* \dagger}+$ H.c. & 152 & I & 304 & I & $P\left\langle u^{\mu} \nabla^{\nu} \chi_{+}\right\rangle D_{\nu} P^{* \dagger}{ }_{\mu}+$ H.c. & 377 & I & 622 & I \\
\hline$i \varepsilon^{\mu \nu \lambda \rho} P^{*}{ }_{\mu} \nabla_{\nu} f_{+\lambda}{ }^{\sigma} u^{\delta} D_{\rho \sigma} P^{* \dagger}{ }_{\delta}+$ H.c. & 153 & & 305 & & $P\left\langle\nabla^{\mu} \chi_{+}\right\rangle u^{\nu} D_{\mu} P_{\nu}^{* \dagger}+$ H.c. & & & 623 & I \\
\hline$i \varepsilon^{\mu \nu \lambda \rho} P^{*}{ }_{\mu} \nabla_{\nu} f_{+\lambda}{ }^{\sigma} u^{\delta} D_{\rho \delta} P^{* \dagger}{ }_{\sigma}+$ H.c. & 154 & & 306 & & $i \varepsilon^{\mu \nu \lambda \rho} P f_{+\mu \nu} \chi_{+} D_{\lambda} P^{* \dagger} \rho+$ H.c. & 378 & & 624 & \\
\hline$i \varepsilon^{\mu \nu \lambda \rho} P_{\mu}^{*} \nabla_{\nu} f_{+\lambda}{ }^{\sigma} u^{\delta} D_{\sigma \delta} P^{* \dagger}{ }_{\rho}+$ H.c. & 155 & I & 307 & I & $i \varepsilon^{\mu \nu \lambda \rho} P \chi_{+} f_{+\mu \nu} D_{\lambda} P_{\rho}^{* \dagger}+$ H.c. & 379 & & 625 & \\
\hline$i \varepsilon^{\mu \nu \lambda \rho} P_{\mu}^{*} \nabla_{\nu} f_{+}{ }^{\sigma \delta} u_{\lambda} D_{\rho \sigma} P^{* \dagger}{ }_{\delta}+$ H.c. & 156 & & 308 & & $i \varepsilon^{\mu \nu \lambda \rho} P\left\langle f_{+\mu \nu}\right\rangle \chi_{+} D_{\lambda} P^{* \dagger} \rho+$ H.c. & 380 & & & \\
\hline$i \varepsilon^{\mu \nu \lambda \rho} P^{*}{ }_{\mu} \nabla_{\nu} f_{+}{ }^{\sigma \delta} u_{\sigma} D_{\lambda \delta} P^{* \dagger}{ }_{\rho}+$ H.c. & 157 & I & 309 & I & $i \varepsilon^{\mu \nu \lambda \rho} P\left\langle f_{+\mu \nu} \chi_{+}\right\rangle D_{\lambda} P_{\rho \dagger}^{* \dagger}+$ H.c. & 381 & & 626 & \\
\hline$i \varepsilon^{\mu \nu \lambda \rho} P_{\mu}^{*} \nabla^{\sigma} f_{+\nu \sigma} u^{\delta} D_{\lambda \delta} P^{* \dagger}{ }_{\rho}+$ H.c. & 158 & I & 310 & I & $i \varepsilon^{\mu \nu \lambda \rho} P\left\langle\chi_{+}\right\rangle f_{+\mu \nu} D_{\lambda} P_{\rho \dagger}^{* \dagger}+$ H.c. & 382 & & 627 & \\
\hline$i \varepsilon^{\mu \nu \lambda \rho} P_{\mu}^{*} f_{+\nu \lambda} f_{-\rho}^{\sigma} P^{* \dagger}{ }_{\sigma}+$ H.c. & 159 & & 311 & & $i P u^{\mu} u^{\nu} \chi_{-} D_{\mu} P_{\nu}^{* \dagger}+$ H.c. & 383 & I & 628 & \\
\hline$i \varepsilon^{\mu \nu \lambda \rho} P^{*}{ }_{\mu} f_{+\nu}{ }^{\sigma} f_{-\lambda \rho} P^{* \dagger}{ }_{\sigma}+$ H.c. & 160 & & 312 & & $i P u^{\mu} u^{\nu} \chi_{-} D_{\nu} P_{\mu}^{* \dagger}+$ H.c. & 384 & & 629 & \\
\hline$i \varepsilon^{\mu \nu \lambda \rho} P_{\mu}^{*} f_{+\nu}{ }^{\sigma} f_{-\lambda \sigma} P_{\rho}^{* \dagger}+$ H.c. & 161 & I & 313 & I & $i P u^{\mu} \chi_{-} u^{\nu} D_{\mu} P_{\nu}^{* \dagger}{ }_{\nu}+$ H.c. & 385 & & 630 & \\
\hline$i \varepsilon^{\mu \nu \lambda \rho} P_{\mu}^{*} f_{+\nu \lambda} f_{-} \sigma \delta D_{\rho \sigma} P^{* \dagger}{ }_{\delta}+$ H.c. & 162 & & 314 & & $i P u^{\mu} \chi_{-} u^{\nu} D_{\nu} P_{\mu}^{* \dagger}+$ H.c. & 386 & & 631 & \\
\hline$i \varepsilon^{\mu \nu \lambda \rho} P_{\mu}^{*} f_{+\nu}^{\sigma} f_{-\lambda}{ }^{\delta} D_{\rho \delta} P^{* \dagger}{ }_{\sigma}+$ H.c. & 163 & & 315 & & $i P \chi_{-} u^{\mu} u^{\nu} D_{\mu} P_{\nu \dagger}^{* \dagger}+$ H.c. & 387 & & 632 & \\
\hline$i \varepsilon^{\mu \nu \lambda \rho} P_{\mu}^{*} f_{+\nu}^{\sigma} f_{-\sigma}{ }^{\delta} D_{\lambda \delta} P^{* \dagger}{ }_{\rho}+$ H.c. & 164 & I & 316 & I & $i P \chi_{-} u^{\mu} u^{\nu} D_{\nu} P_{\mu}^{* \dagger}+$ H.c. & & & 633 & \\
\hline$i \varepsilon^{\mu \nu \lambda \rho} P_{\mu}^{*} f_{+\nu \lambda} h_{\rho}{ }^{\sigma} P^{* \dagger}{ }_{\sigma}+$ H.c. & 165 & I & 317 & I & $i P\left\langle u^{\mu} u^{\nu}\right\rangle \chi_{-} D_{\mu} P_{\nu}^{* \dagger}+$ H.c. & & & 634 & I \\
\hline$i \varepsilon^{\mu \nu \lambda \rho} P_{\mu}^{*} f_{+\nu}{ }^{\sigma} h_{\lambda \sigma} P^{* \dagger}{ }_{\rho}+$ H.c. & 166 & I & 318 & I & $i P\left\langle u^{\mu} u^{\nu} \chi_{-}\right\rangle D_{\mu} P_{\nu \dagger}^{* \dagger}+$ H.c. & 388 & I & 635 & \\
\hline$i \varepsilon^{\mu \nu \lambda \rho} P_{\mu}^{*} f_{+\nu \lambda} h^{\sigma \delta} D_{\rho \sigma} P^{* \dagger}{ }_{\delta}+$ H.c. & 167 & & 319 & & $i P\left\langle u^{\mu} \chi_{-}\right\rangle u^{\nu} D_{\nu} P_{\mu}^{* \dagger}+$ H.c. & & & 636 & I \\
\hline$i \varepsilon^{\mu \nu \lambda \rho} P_{\mu}^{*} f_{+\nu \lambda} h^{\sigma \delta} D_{\sigma \delta} P^{* \dagger}{ }_{\rho}+$ H.c. & 168 & I & 320 & I & $i P\left\langle\chi_{-}\right\rangle u^{\mu} u^{\nu} D_{\mu} P_{\nu}^{* \dagger}+$ H.c. & & & 637 & \\
\hline$i \varepsilon^{\mu \nu \lambda \rho} P_{\mu}^{*} f_{+\nu}{ }^{\sigma} h_{\lambda}{ }^{\delta} D_{\rho \delta} P^{* \dagger}{ }_{\sigma}+$ H.c. & 169 & & 321 & & $i P\left\langle u^{\mu} u^{\nu} \chi_{-}\right\rangle D_{\nu} P_{\mu}^{* \dagger}+$ H.c. & & & 638 & \\
\hline
\end{tabular}




\begin{tabular}{|c|c|c|c|c|c|c|c|c|c|}
\hline$O_{n}$ & $S U(2)$ & $\tilde{c}_{n}^{(4)}$ & $S U(3)$ & $\tilde{C}_{n}^{(4)}$ & $O_{n}$ & $S U(2)$ & $\tilde{c}_{n}^{(4)}$ & $S U(3)$ & $\tilde{C}_{n}^{(4)}$ \\
\hline$\overline{i \varepsilon^{\mu \nu \lambda \rho} P^{*}{ }_{\mu} f_{+\nu}{ }^{\sigma} h_{\sigma}{ }^{\delta} D_{\lambda \delta} P^{* \dagger}{ }_{\rho}+\text { H.c. }}$ & 170 & I & 322 & I & $i P\left\langle u^{\mu} \chi_{-}\right\rangle u^{\nu} D_{\mu} P^{* \dagger}{ }_{\nu}+$ H.c. & & & 639 & I \\
\hline$i \varepsilon^{\mu \nu \lambda \rho} P_{\mu}^{*}\left\langle\nabla_{\nu} f_{+\lambda}{ }^{\sigma}\right\rangle u_{\rho} P^{* \dagger}{ }_{\sigma}{ }^{\prime}+$ H.c. & 171 & I & & & $i P\left\langle\chi_{-}\right\rangle u^{\mu} u^{\nu} D_{\nu} P^{* \dagger}{ }_{\mu}+$ H.c. & & & 640 & \\
\hline$i \varepsilon^{\mu \nu \lambda \rho} P^{*}{ }_{\mu}^{\mu}\left\langle\nabla_{\nu} f_{+\lambda}{ }^{\sigma}\right\rangle u_{\sigma} P^{* \dagger} \rho$ & 172 & $\mathrm{I}$ & & & $i \varepsilon^{\mu \nu \lambda \rho} P f_{-\mu \nu} \chi_{-} D_{\lambda} P^{\mu \dagger \dagger} \rho+$ H.c. & 389 & & 641 & \\
\hline$i \varepsilon^{\mu \nu \lambda \rho} P^{*}{ }_{\mu}\left\langle\nabla_{\nu} f_{+\lambda}{ }^{\sigma}\right\rangle u^{\delta} D_{\rho \sigma} P^{* \dagger}{ }_{\delta}+$ H.c. & 173 & I & & & $i \varepsilon^{\mu \nu \lambda \rho} P \chi_{-} f_{-\mu \nu} D_{\lambda} P_{\rho}^{* \dagger}+$ H.c. & 390 & & 642 & \\
\hline$i \varepsilon^{\mu \nu \lambda \rho} P^{*}{ }_{\mu}\left\langle\nabla_{\nu} f_{+\lambda}{ }^{\sigma}\right\rangle u^{\delta} D_{\rho \delta} P^{* \dagger}{ }_{\sigma}+$ H.c. & 174 & I & & & $i \varepsilon^{\mu \nu \lambda \rho} P u_{\mu} \nabla_{\nu} \chi_{-} D_{\lambda} P_{\rho}^{* \dagger}+$ H.c. & 391 & & 643 & \\
\hline$i \varepsilon^{\mu \nu \lambda \rho} P_{\mu}^{*}\left\langle\nabla_{\nu} f_{+\lambda}{ }^{\sigma}\right\rangle u^{\delta} D_{\sigma \delta} P^{* \dagger} \rho$ & 175 & I & & & $i \varepsilon^{\mu \nu \lambda \rho} P \nabla_{\mu} \chi_{-} u_{\nu} D_{\lambda} P^{* \dagger} \rho+$ H.c. & 392 & & 644 & \\
\hline$i \varepsilon^{\mu \nu \lambda \rho} P^{*}{ }_{\mu}\left\langle f_{+\nu \lambda}\right\rangle f_{-\rho}{ }^{\sigma} P^{* \dagger}{ }_{\sigma}+$ H.c. & 176 & I & & & $i \varepsilon^{\mu \nu \lambda \rho} P\left\langle f_{-\mu \nu} \chi_{-}\right\rangle D_{\lambda} P^{* \dagger} \rho+$ H.c. & 393 & I & 645 & I \\
\hline$i \varepsilon^{\mu \nu \lambda \rho} P_{\mu}^{*}\left\langle f_{+\nu \lambda}\right\rangle h_{\rho}{ }^{\sigma} P^{* \dagger}{ }_{\sigma}+$ H.c. & 177 & I & & & $i \varepsilon^{\mu \nu \lambda \rho} P\left\langle\chi_{-}\right\rangle f_{-\mu \nu} D_{\lambda} P^{* \dagger} \rho+$ H.c. & & & 646 & I \\
\hline$i \varepsilon^{\mu \nu \lambda \rho} P^{*}\left\langle f_{+\nu \lambda}\right\rangle h^{\sigma \delta} D_{\rho \sigma} P^{* \dagger}{ }_{\delta}+$ H.c. & 178 & I & & & $i \varepsilon^{\mu \nu \lambda \rho} P\left\langle u_{\mu} \nabla_{\nu} \chi_{-}\right\rangle D_{\lambda} P^{* \dagger} \rho+$ H.c. & 394 & I & 647 & I \\
\hline$i \varepsilon^{\mu \nu \lambda \rho} P^{*}\left\langle f_{+\nu \lambda}\right\rangle h^{\sigma \delta} D_{\sigma \delta} P^{* \dagger} \rho$ & 179 & I & & & $i \varepsilon^{\mu \nu \lambda \rho} P\left\langle\nabla_{\mu} \chi_{-}\right\rangle u_{\nu} D_{\lambda} P^{* \dagger}{ }_{\rho}+$ H.c. & & & 648 & I \\
\hline$i \varepsilon^{\mu \nu \lambda \rho} P_{\mu}^{*}\left\langle\nabla_{\nu} f_{+\lambda}{ }^{\sigma} u_{\rho}\right\rangle P^{* \dagger}{ }_{\sigma}+$ H.c. & & & 323 & I & $i P \nabla^{\mu} \nabla^{\nu} \chi_{-} D_{\mu} P^{* \dagger}{ }_{\nu}+$ H.c. & 395 & I & 649 & I \\
\hline$i \varepsilon^{\mu \nu \lambda \rho} P^{*}{ }_{\mu}\left\langle\nabla_{\nu} f_{+\lambda}{ }^{\sigma} u_{\sigma}\right\rangle P^{* \dagger} \rho$ & & & 324 & I & $i P\left\langle\nabla^{\mu} \nabla^{\nu} \chi_{-}\right\rangle D_{\mu} P^{* \dagger}{ }_{\nu}+$ H.c. & 396 & I & 650 & I \\
\hline$i \varepsilon^{\mu \nu \lambda \rho} P^{*}{ }_{\mu}\left\langle\nabla_{\nu} f_{+\lambda}{ }^{\sigma} u^{\delta}\right\rangle D_{\rho \sigma} P^{* \dagger}{ }_{\delta}+$ H.c. & & & 325 & I & $P f_{+}{ }^{\mu \nu} \chi_{-} D_{\mu} P^{* \dagger}{ }_{\nu}+$ H.c. & 397 & & 651 & \\
\hline$i \varepsilon^{\mu \nu \lambda \rho} P^{*}{ }_{\mu}\left\langle\nabla_{\nu} f_{+\lambda}{ }^{\sigma} u^{\delta}\right\rangle D_{\rho \delta} P^{* \dagger}{ }_{\sigma}+$ H.c. & & & 326 & I & $P \chi_{-} f_{+}{ }^{\mu \nu} D_{\mu} P^{* \dagger}{ }_{\nu}+$ H.c. & 398 & & 652 & \\
\hline$i \varepsilon^{\mu \nu \lambda \rho} P_{\mu}^{*}\left\langle\nabla_{\nu} f_{+\lambda}{ }^{\sigma} u^{\delta}\right\rangle D_{\sigma \delta} P^{* \dagger}{ }_{\rho}$ & & & 327 & I & $P\left\langle f_{+}{ }^{\mu \nu}\right\rangle \chi_{-} D_{\mu} P^{* \dagger}{ }_{\nu}+$ H.c. & 399 & & & \\
\hline$i \varepsilon^{\mu \nu \lambda \rho} P_{\mu}^{*}\left\langle f_{+\nu \lambda} f_{-\rho}{ }^{\sigma}\right\rangle P^{* \dagger}{ }_{\sigma}+$ H.c. & & & 328 & I & $P\left\langle f_{+}{ }^{\mu \nu} \chi_{-}\right\rangle D_{\mu} P^{* \dagger}{ }_{\nu}+$ H.c. & 400 & & 653 & \\
\hline$i \varepsilon^{\mu \nu \lambda \rho} P^{*}{ }_{\mu}\left\langle f_{+\nu \lambda} h_{\rho}{ }^{\sigma}\right\rangle P^{* \dagger}{ }_{\sigma}+$ H.c. & & & 329 & I & $P\left\langle\chi_{-}\right\rangle f_{+}^{\mu \nu} D_{\mu}^{\mu} P_{\nu}^{* \dagger}+$ H.c. & 401 & & 654 & \\
\hline$i \varepsilon^{\mu \nu \lambda \rho} P_{\mu}^{*}\left\langle f_{+\nu \lambda} h^{\sigma \delta}\right\rangle D_{\rho \sigma} P^{* \dagger} \delta+$ H.c. & & & 330 & I & $i \varepsilon^{\mu \nu \lambda \rho} P\left\langle D_{\mu} D^{\sigma} F_{L \nu \sigma}\right\rangle D_{\lambda} P_{\rho}^{* \dagger}+$ P. ++ H.c. & 402 & & & \\
\hline$i \varepsilon^{\mu \nu \lambda \rho} P^{*}\left\langle f_{+\nu \lambda} h^{\sigma \delta}\right\rangle D_{\sigma \delta} P_{\rho}^{* \dagger} \rho$ & & & 331 & I & $i \varepsilon^{\mu \nu \lambda \rho} P\left\langle D_{\mu}^{\mu} D^{\sigma} F_{L \nu}{ }^{\delta}\right\rangle D_{\lambda \sigma \delta} P^{* \dagger} \rho+$ P. ++ H.c. & 403 & & & \\
\hline$i P^{* \mu} \nabla_{\mu} \nabla^{\nu} f_{+\nu}^{\lambda} P_{\lambda}^{* \dagger}{ }_{\lambda}+$ H.c. & 180 & I & 332 & I & $i P\left\langle F_{L}^{\mu \nu} F_{L \mu}{ }^{\lambda}\right\rangle D_{\nu} P_{\lambda}^{* \dagger}{ }_{\lambda}+$ P. + H.c. & 404 & & 655 & \\
\hline$i P^{* \mu} \nabla^{\nu} \nabla_{\nu} f_{+\mu}^{\lambda} P^{* \dagger} \lambda$ & 181 & I & 333 & I & & & & & \\
\hline
\end{tabular}

The long relations in the sixth column of Table VII are

$\tilde{C}_{10}^{(3)}=-D_{2}^{(3)}-D_{6}^{(3)}+D_{9}^{(3)}, \quad \tilde{C}_{11}^{(3)}=D_{2}^{(3)}+D_{4}^{(3)}+D_{6}^{(3)}+D_{8}^{(3)}, \quad \tilde{C}_{13}^{(3)}=-2 D_{1}^{(3)}-D_{2}^{(3)}-3 D_{6}^{(3)}$,

$\tilde{C}_{14}^{(3)}=D_{2}^{(3)}+D_{6}^{(3)}+D_{9}^{(3)}, \quad \tilde{C}_{15}^{(3)}=2 D_{3}^{(3)}+D_{4}^{(3)}+3 D_{8}^{(3)}, \quad \tilde{C}_{18}^{(3)}=2 D_{5}^{(3)}-D_{6}^{(3)}-D_{8}^{(3)}$,

$\tilde{C}_{53}^{(3)}=\tilde{C}_{55}^{(3)}=-2 D_{3}^{(3)}-2 D_{7}^{(3)}-D_{17}^{(3)}, \quad \tilde{C}_{54}^{(3)}=-2 D_{4}^{(3)}-2 D_{7}^{(3)}+2 D_{17}^{(3)}$.

TABLE X. The $\mathcal{O}\left(p^{4}\right)$ order results in the HQ limit. When a term $P_{n}$ is not given a label in the 2 nd or 5 th ( 3 rd or 6 th) column, it is not independent and can be expressed with terms having a label in the 2nd and 5th (3rd and 6th) columns.

\begin{tabular}{|c|c|c|c|c|c|}
\hline$P_{n}$ & $S U(2)$ & $S U(3)$ & $P_{n}$ & $S U(2)$ & $S U(3)$ \\
\hline$\overline{\left\langle H\left\langle u^{\mu} u_{\mu}\right\rangle u^{\nu} u_{\nu} \bar{H}\right\rangle}$ & 1 & 1 & $\left\langle H\left\langle f_{+}{ }^{\mu \nu} u^{\lambda}\right\rangle u^{\rho} \sigma_{\mu \rho} v_{\nu} v_{\lambda} \bar{H}\right\rangle$ & 72 & \\
\hline$\left\langle H\left\langle u^{\mu} u^{\nu}\right\rangle u_{\mu} u_{\nu} \bar{H}\right\rangle$ & 2 & 2 & $\left\langle H \nabla^{\mu} \nabla_{\mu} \chi_{+} \bar{H}\right\rangle$ & & 111 \\
\hline$\left\langle H\left\langle u^{\mu} u_{\mu}\right\rangle u^{\nu} u^{\lambda} v_{\nu} v_{\lambda} \bar{H}\right\rangle$ & 3 & 3 & $\left\langle H \nabla^{\mu} \nabla^{\nu} \chi_{+} v_{\mu} v_{\nu} \bar{H}\right\rangle$ & & 112 \\
\hline$\left\langle H\left\langle u^{\mu} u^{\nu}\right\rangle u_{\mu} u^{\lambda} v_{\nu} v_{\lambda} \bar{H}\right\rangle+$ H.c. & 4 & 4 & $\left\langle H\left\langle\nabla^{\mu} \nabla_{\mu} \chi_{+}\right\rangle \bar{H}\right\rangle$ & & 113 \\
\hline$\left\langle H\left\langle u^{\mu} u^{\nu}\right\rangle u^{\lambda} u_{\lambda} v_{\mu} v_{\nu} \bar{H}\right\rangle$ & & 5 & $\left\langle H\left\langle\nabla^{\mu} \nabla^{\nu} \chi_{+}\right\rangle v_{\mu} v_{\nu} \bar{H}\right\rangle$ & & 114 \\
\hline$\left\langle H\left\langle u^{\mu} u^{\nu}\right\rangle u^{\lambda} u^{\rho} v_{\mu} v_{\nu} v_{\lambda} v_{\rho} \bar{H}\right\rangle$ & 5 & 6 & $\left\langle H\left\langle f_{+}{ }^{\mu \nu} u_{\mu} u^{\lambda}\right\rangle \sigma_{\nu \lambda} \bar{H}\right\rangle+$ H.c. & & 115 \\
\hline$\left\langle H\left\langle u^{\mu} u_{\mu} u^{\nu}\right\rangle u_{\nu} \bar{H}\right\rangle$ & & 7 & $\left\langle H\left\langle f_{+}{ }^{\mu \nu} u^{\lambda} u_{\lambda}\right\rangle \sigma_{\mu \nu} \bar{H}\right\rangle$ & & 116 \\
\hline$\left\langle H\left\langle u^{\mu} u_{\mu} u^{\nu}\right\rangle u^{\lambda} v_{\nu} v_{\lambda} \bar{H}\right\rangle$ & & 8 & $\left\langle H\left\langle f_{+}{ }^{\mu \nu} u^{\lambda} u^{\rho}\right\rangle \sigma_{\mu \nu} v_{\lambda} v_{\rho} \bar{H}\right\rangle$ & & 117 \\
\hline$\left\langle H\left\langle u^{\mu} u^{\nu} u^{\lambda}\right\rangle u_{\mu} v_{\nu} v_{\lambda} \bar{H}\right\rangle$ & & 9 & $\left\langle H\left\langle f_{+}{ }^{\mu \nu} u^{\lambda} u^{\rho}\right\rangle \sigma_{\mu \lambda} v_{\nu} v_{\rho} \bar{H}\right\rangle+$ H.c. & & 118 \\
\hline$\left\langle H\left\langle u^{\mu} u^{\nu} u^{\lambda}\right\rangle u^{\rho} v_{\mu} v_{\nu} v_{\lambda} v_{\rho} \bar{H}\right\rangle$ & & 10 & $i\left\langle H u^{\mu} f_{+\mu}^{\nu} u_{\nu} \bar{H}\right\rangle$ & & 119 \\
\hline$\left\langle H\left\langle u^{\mu} u_{\mu} u^{\nu} u_{\nu}\right\rangle \bar{H}\right\rangle$ & & 11 & $i\left\langle H u^{\mu} f_{+\mu}{ }^{\nu} u^{\lambda} v_{\nu} v_{\lambda} \bar{H}\right\rangle+$ H.c. & & 120 \\
\hline$\left\langle H\left\langle u^{\mu} u^{\nu} u_{\mu} u_{\nu}\right\rangle \bar{H}\right\rangle$ & & 12 & $\left\langle H\left\langle u^{\mu} u_{\mu}\right\rangle f_{+}{ }^{\nu \lambda} \sigma_{\nu \lambda} \bar{H}\right\rangle$ & 73 & 121 \\
\hline
\end{tabular}


TABLE X. (Continued)

\begin{tabular}{|c|c|c|c|c|c|}
\hline$P_{n}$ & $S U(2)$ & $S U(3)$ & $P_{n}$ & $S U(2)$ & $S U(3)$ \\
\hline$\left\langle H\left\langle u^{\mu} u_{\mu} u^{\nu} u^{\lambda}\right\rangle v_{\nu} v_{\lambda} \bar{H}\right\rangle$ & & 13 & $\left\langle H\left\langle u^{\mu} u^{\nu}\right\rangle f_{+\mu}^{\lambda} \sigma_{\nu \lambda} \bar{H}\right\rangle$ & 74 & 122 \\
\hline$\left\langle H\left\langle u^{\mu} u^{\nu} u_{\mu} u^{\lambda}\right\rangle v_{\nu} v_{\lambda} \bar{H}\right\rangle$ & & 14 & $\left\langle H\left\langle u^{\mu} u^{\nu}\right\rangle f_{+}{ }^{\lambda \rho} \sigma_{\mu \lambda} v_{\nu} v_{\rho} \bar{H}\right\rangle$ & 75 & 123 \\
\hline$\left\langle H\left\langle u^{\mu} u^{\nu} u^{\lambda} u^{\rho}\right\rangle v_{\mu} v_{\nu} v_{\lambda} v_{\rho} \bar{H}\right\rangle$ & & 15 & $\left\langle H\left\langle u^{\mu} u^{\nu}\right\rangle f_{+}^{\lambda \rho} \sigma_{\lambda \rho} v_{\mu} v_{\nu} \bar{H}\right\rangle$ & 76 & 124 \\
\hline$i\left\langle H\left\langle u^{\mu} u_{\mu}\right\rangle u^{\nu} u^{\lambda} \sigma_{\nu \lambda} \bar{H}\right\rangle$ & 6 & 16 & $\left\langle H f_{+}{ }^{\mu \nu} u_{\mu} u^{\lambda} \sigma_{\nu \lambda} \bar{H}\right\rangle+$ H.c. & & 125 \\
\hline$i\left\langle H\left\langle u^{\mu} u^{\nu}\right\rangle u_{\mu} u^{\lambda} \sigma_{\nu \lambda} \bar{H}\right\rangle+$ H.c. & 7 & 17 & $\left\langle H f_{+}{ }^{\mu \nu} u^{\lambda} u_{\mu} \sigma_{\nu \lambda} \bar{H}\right\rangle+$ H.c. & & 126 \\
\hline$i\left\langle H\left\langle u^{\mu} u^{\nu}\right\rangle u^{\lambda} u^{\rho} \sigma_{\mu \lambda} v_{\nu} v_{\rho} \bar{H}\right\rangle+$ H.c. & 8 & 18 & $\left\langle H f_{+}{ }^{\mu \nu} u^{\lambda} u_{\lambda} \sigma_{\mu \nu} \bar{H}\right\rangle+$ H.c. & & 127 \\
\hline$i\left\langle H\left\langle u^{\mu} u^{\nu}\right\rangle u^{\lambda} u^{\rho} \sigma_{\lambda \rho} v_{\mu} v_{\nu} \bar{H}\right\rangle$ & 9 & 19 & $\left\langle H f_{+}{ }^{\mu \nu} u^{\lambda} u^{\rho} \sigma_{\mu \nu} v_{\lambda} v_{\rho} \bar{H}\right\rangle+$ H.c. & & 128 \\
\hline$i\left\langle H\left\langle f_{+}{ }^{\mu \nu}\right\rangle u_{\mu} u_{\nu} \bar{H}\right\rangle$ & 10 & & $\left\langle H f_{+}{ }^{\mu \nu} u^{\lambda} u^{\rho} \sigma_{\mu \lambda} v_{\nu} v_{\rho} \bar{H}\right\rangle+$ H.c. & & 129 \\
\hline$i\left\langle H\left\langle f_{+}{ }^{\mu \nu}\right\rangle u_{\mu} u^{\lambda} v_{\nu} v_{\lambda} \bar{H}\right\rangle+$ H.c. & 11 & & $\left\langle H f_{+}{ }^{\mu \nu} u^{\lambda} u^{\rho} \sigma_{\mu \rho} v_{\nu} v_{\lambda} \bar{H}\right\rangle+$ H.c. & & 130 \\
\hline$i\left\langle H\left\langle u^{\mu} u^{\nu} u^{\lambda}\right\rangle u_{\mu} \sigma_{\nu \lambda} \bar{H}\right\rangle$ & & 20 & $\left\langle H f_{+}{ }^{\mu \nu} f_{+\mu \nu} \bar{H}\right\rangle$ & 77 & 131 \\
\hline$i\left\langle H\left\langle u^{\mu} u^{\nu} u^{\lambda}\right\rangle u^{\rho} \sigma_{\mu \nu} v_{\lambda} v_{\rho} \bar{H}\right\rangle$ & & 21 & $\left\langle H f_{+}{ }^{\mu \nu} f_{+\mu}{ }^{\lambda} v_{\nu} v_{\lambda} \bar{H}\right\rangle$ & 78 & 132 \\
\hline$i\left\langle H\left\langle u^{\mu} u_{\mu} u^{\nu} u^{\lambda}\right\rangle \sigma_{\nu \lambda} \bar{H}\right\rangle$ & & 22 & $i\left\langle H f_{+}{ }^{\mu \nu} f_{+\mu}{ }^{\lambda} \sigma_{\nu \lambda} \bar{H}\right\rangle$ & 79 & 133 \\
\hline$i\left\langle H\left\langle u^{\mu} u^{\nu} u^{\lambda} u^{\rho}\right\rangle \sigma_{\mu \nu} v_{\lambda} v_{\rho} \bar{H}\right\rangle$ & & 23 & $i\left\langle H f_{+}{ }^{\mu \nu} f_{+}{ }^{\lambda \rho} \sigma_{\mu \lambda} v_{\nu} v_{\rho} \bar{H}\right\rangle$ & 80 & 134 \\
\hline$\left\langle H u^{\mu} u_{\mu} u^{\nu} u_{\nu} \bar{H}\right\rangle$ & & 24 & $\left\langle H\left\langle f_{+}{ }^{\mu \nu}\right\rangle f_{+\mu \nu} \bar{H}\right\rangle$ & 81 & \\
\hline$\left\langle H u^{\mu} u^{\nu} u_{\mu} u_{\nu} \bar{H}\right\rangle$ & & 25 & $\left\langle H\left\langle f_{+}{ }^{\mu \nu}\right\rangle f_{+\mu}{ }^{\lambda} v_{\nu} v_{\lambda} \bar{H}\right\rangle$ & 82 & \\
\hline$\left\langle H u^{\mu} u_{\mu} u^{\nu} u^{\lambda} v_{\nu} v_{\lambda} \bar{H}\right\rangle+$ H.c. & & 26 & $\left\langle H\left\langle f_{+}{ }^{\mu \nu} f_{+\mu \nu}\right\rangle \bar{H}\right\rangle$ & & 135 \\
\hline$\left\langle H u^{\mu} u^{\nu} u_{\mu} u^{\lambda} v_{\nu} v_{\lambda} \bar{H}\right\rangle+$ H.c. & & 27 & $\left\langle H\left\langle f_{+}{ }^{\mu \nu} f_{+\mu}{ }^{\lambda}\right\rangle v_{\nu} v_{\lambda} \bar{H}\right\rangle$ & & 136 \\
\hline$i\left\langle H u^{\mu} u_{\mu} u^{\nu} u^{\lambda} \sigma_{\nu \lambda} \bar{H}\right\rangle+$ H.c. & & 28 & $\left\langle H u^{\mu} u_{\mu} \chi_{+} \bar{H}\right\rangle+$ H.c. & 83 & 137 \\
\hline$i\left\langle H u^{\mu} u^{\nu} u_{\mu} u^{\lambda} \sigma_{\nu \lambda} \bar{H}\right\rangle+$ H.c. & & 29 & $\left\langle H u^{\mu} \chi_{+} u_{\mu} \bar{H}\right\rangle$ & 84 & 138 \\
\hline$i\left\langle H u^{\mu} u^{\nu} u^{\lambda} u^{\rho} \sigma_{\mu \nu} v_{\lambda} v_{\rho} \bar{H}\right\rangle+$ H.c. & & 30 & $\left\langle H u^{\mu} u^{\nu} \chi_{+} v_{\mu} v_{\nu} \bar{H}\right\rangle+$ H.c. & 85 & 139 \\
\hline$i\left\langle H u^{\mu} u^{\nu} u^{\lambda} u^{\rho} \sigma_{\mu \lambda} v_{\nu} v_{\rho} \bar{H}\right\rangle+$ H.c. & & 31 & $\left\langle H u^{\mu} \chi_{+} u^{\nu} v_{\mu} v_{\nu} \bar{H}\right\rangle$ & 86 & 140 \\
\hline$i\left\langle H\left\langle f_{+}{ }^{\mu \nu} u_{\mu} u_{\nu}\right\rangle \bar{H}\right\rangle$ & 12 & 32 & $i\left\langle H u^{\mu} u^{\nu} \chi_{+} \sigma_{\mu \nu} \bar{H}\right\rangle+$ H.c. & 87 & 141 \\
\hline$i\left\langle H\left\langle f_{+}{ }^{\mu \nu} u_{\mu} u^{\lambda}\right\rangle v_{\nu} v_{\lambda} \bar{H}\right\rangle+$ H.c. & 13 & 33 & $i\left\langle H u^{\mu} \chi_{+} u^{\nu} \sigma_{\mu \nu} \bar{H}\right\rangle$ & 88 & 142 \\
\hline$\left\langle H\left\langle f_{+}{ }^{\mu \nu}\right\rangle u_{\mu} u^{\lambda} \sigma_{\nu \lambda} \bar{H}\right\rangle+$ H.c. & 14 & & $\left\langle H\left\langle u^{\mu} u_{\mu}\right\rangle \chi_{+} \bar{H}\right\rangle$ & & 143 \\
\hline$\left\langle H\left\langle f_{+}{ }^{\mu \nu}\right\rangle u^{\lambda} u_{\lambda} \sigma_{\mu \nu} \bar{H}\right\rangle$ & 15 & & $\left\langle H\left\langle u^{\mu} u_{\mu} \chi_{+}\right\rangle \bar{H}\right\rangle$ & 89 & 144 \\
\hline$\left\langle H\left\langle f_{+}{ }^{\mu \nu}\right\rangle u^{\lambda} u^{\rho} \sigma_{\mu \nu} v_{\lambda} v_{\rho} \bar{H}\right\rangle$ & 16 & & $\left\langle H\left\langle u^{\mu} \chi_{+}\right\rangle u_{\mu} \bar{H}\right\rangle$ & & 145 \\
\hline$\left\langle H\left\langle f_{+}{ }^{\mu \nu}\right\rangle u^{\lambda} u^{\rho} \sigma_{\mu \lambda} v_{\nu} v_{\rho} \bar{H}\right\rangle+$ H.c. & 17 & & $\left\langle H\left\langle\chi_{+}\right\rangle u^{\mu} u_{\mu} \bar{H}\right\rangle$ & & 146 \\
\hline$\left\langle H\left\langle f_{+}{ }^{\mu \nu}\right\rangle h^{\lambda \rho} \gamma_{5} \gamma_{\mu} v_{\nu} v_{\lambda} v_{\rho} \bar{H}\right\rangle$ & 18 & & $\left\langle H\left\langle u^{\mu} u^{\nu}\right\rangle \chi_{+} v_{\mu} v_{\nu} \bar{H}\right\rangle$ & & 147 \\
\hline$i\left\langle H f_{+}{ }^{\mu \nu} u_{\mu} u_{\nu} \bar{H}\right\rangle+$ H.c. & & 34 & $\left\langle H\left\langle u^{\mu} u^{\nu} \chi_{+}\right\rangle v_{\mu} v_{\nu} \bar{H}\right\rangle$ & 90 & 148 \\
\hline$i\left\langle H f_{+}{ }^{\mu \nu} u_{\mu} u^{\lambda} v_{\nu} v_{\lambda} \bar{H}\right\rangle+$ H.c. & & 35 & $\left\langle H\left\langle u^{\mu} \chi_{+}\right\rangle u^{\nu} v_{\mu} v_{\nu} \bar{H}\right\rangle$ & & 149 \\
\hline$i\left\langle H f_{+}{ }^{\mu \nu} u^{\lambda} u_{\mu} v_{\nu} v_{\lambda} \bar{H}\right\rangle+$ H.c. & & 36 & $\left\langle H\left\langle\chi_{+}\right\rangle u^{\mu} u^{\nu} v_{\mu} v_{\nu} \bar{H}\right\rangle$ & & 150 \\
\hline$\left\langle H\left\langle f_{+}{ }^{\mu \nu} u_{\mu}\right\rangle u^{\lambda} \sigma_{\nu \lambda} \bar{H}\right\rangle$ & & 37 & $i\left\langle H\left\langle u^{\mu} u^{\nu} \chi_{+}\right\rangle \sigma_{\mu \nu} \bar{H}\right\rangle$ & & 151 \\
\hline$\left\langle H\left\langle f_{+}{ }^{\mu \nu} u^{\lambda}\right\rangle u_{\mu} \sigma_{\nu \lambda} \bar{H}\right\rangle$ & & 38 & $i\left\langle H\left\langle\chi_{+}\right\rangle u^{\mu} u^{\nu} \sigma_{\mu \nu} \bar{H}\right\rangle$ & & 152 \\
\hline$\left\langle H\left\langle f_{+}{ }^{\mu \nu} u^{\lambda}\right\rangle u_{\lambda} \sigma_{\mu \nu} \bar{H}\right\rangle$ & & 39 & $\left\langle H f_{+}{ }^{\mu \nu} \chi_{+} \sigma_{\mu \nu} \bar{H}\right\rangle+$ H.c. & 91 & 153 \\
\hline$\left\langle H\left\langle f_{+}{ }^{\mu \nu} u^{\lambda}\right\rangle u^{\rho} \sigma_{\mu \nu} v_{\lambda} v_{\rho} \bar{H}\right\rangle$ & & 40 & $\left\langle H\left\langle f_{+}{ }^{\mu \nu}\right\rangle \chi_{+} \sigma_{\mu \nu} \bar{H}\right\rangle$ & 92 & \\
\hline$\left\langle H\left\langle f_{+}{ }^{\mu \nu} u^{\lambda}\right\rangle u^{\rho} \sigma_{\mu \lambda} v_{\nu} v_{\rho} \bar{H}\right\rangle$ & & 41 & $\left\langle H\left\langle f_{+}{ }^{\mu \nu} \chi_{+}\right\rangle \sigma_{\mu \nu} \bar{H}\right\rangle$ & 93 & 154 \\
\hline$\left\langle H\left\langle f_{+}{ }^{\mu \nu} u^{\lambda}\right\rangle u^{\rho} \sigma_{\mu \rho} v_{\nu} v_{\lambda} \bar{H}\right\rangle$ & & 42 & $\left\langle H\left\langle\chi_{+}\right\rangle f_{+}{ }^{\mu \nu} \sigma_{\mu \nu} \bar{H}\right\rangle$ & 94 & 155 \\
\hline$\left\langle H f_{+}{ }^{\mu \nu} h^{\lambda \rho} \gamma_{5} \gamma_{\mu} v_{\nu} v_{\lambda} v_{\rho} \bar{H}\right\rangle+$ H.c. & & 43 & $\left\langle H \chi_{+}^{2} \bar{H}\right\rangle$ & 95 & 156 \\
\hline$\left\langle H \nabla^{\mu} f_{+\mu}{ }^{\nu} u^{\lambda} \gamma_{5} \gamma_{\nu} v_{\lambda} \bar{H}\right\rangle+$ H.c. & & 44 & $\left\langle H \nabla^{\mu} \nabla_{\mu} \chi_{+} \bar{H}\right\rangle$ & 96 & \\
\hline$\left\langle H \nabla^{\mu} f_{+\mu}{ }^{\nu} u^{\lambda} \gamma_{5} \gamma_{\lambda} v_{\nu} \bar{H}\right\rangle+$ H.c. & & 45 & $\left\langle H \nabla^{\mu} \nabla^{\nu} \chi_{+} v_{\mu} v_{\nu} \bar{H}\right\rangle$ & 97 & \\
\hline$\left\langle H \nabla^{\mu} f_{+}{ }^{\nu \lambda} u_{\mu} \gamma_{5} \gamma_{\nu} v_{\lambda} \bar{H}\right\rangle+$ H.c. & & 46 & $\left\langle H\left\langle\nabla^{\mu} \nabla_{\mu} \chi_{+}\right\rangle \bar{H}\right\rangle$ & 98 & \\
\hline$\left\langle H \nabla^{\mu} f_{+}{ }^{\nu \lambda} u_{\nu} \gamma_{5} \gamma_{\mu} v_{\lambda} \bar{H}\right\rangle+$ H.c. & & 47 & $\left\langle H\left\langle\nabla^{\mu} \nabla^{\nu} \chi_{+}\right\rangle v_{\mu} v_{\nu} \bar{H}\right\rangle$ & 99 & \\
\hline$\left\langle H \nabla^{\mu} f_{+}{ }^{\nu \lambda} u^{\rho} \gamma_{5} \gamma_{\nu} v_{\mu} v_{\lambda} v_{\rho} \bar{H}\right\rangle+$ H.c. & & 48 & $\left\langle H\left\langle\chi_{+}\right\rangle \chi_{+} \bar{H}\right\rangle$ & & 157 \\
\hline$\left\langle H\left\langle f_{+}{ }^{\mu \nu} f_{-\mu}{ }^{\lambda}\right\rangle \gamma_{5} \gamma_{\nu} v_{\lambda} \bar{H}\right\rangle$ & & 49 & $\left\langle H\left\langle\chi_{+}^{2}\right\rangle \bar{H}\right\rangle$ & 100 & 158 \\
\hline$\left\langle H\left\langle f_{+}{ }^{\mu \nu} h_{\mu}{ }^{\lambda}\right\rangle \gamma_{5} \gamma_{\nu} v_{\lambda} \bar{H}\right\rangle$ & & 50 & $\left\langle H\left\langle\chi_{+}\right\rangle\left\langle\chi_{+}\right\rangle \bar{H}\right\rangle$ & & 159 \\
\hline$\left\langle H\left\langle f_{+}{ }^{\mu \nu} h_{\mu}{ }^{\lambda}\right\rangle \gamma_{5} \gamma_{\lambda} v_{\nu} \bar{H}\right\rangle$ & & 51 & $\left\langle H u^{\mu} u^{\nu} \chi_{-} \gamma_{5} \gamma_{\mu} v_{\nu} \bar{H}\right\rangle+$ H.c. & 101 & 160 \\
\hline$\left\langle H\left\langle f_{+}{ }^{\mu \nu} h^{\lambda \rho}\right\rangle \gamma_{5} \gamma_{\mu} v_{\nu} v_{\lambda} v_{\rho} \bar{H}\right\rangle$ & & 52 & $\left\langle H u^{\mu} u^{\nu} \chi_{-} \gamma_{5} \gamma_{\nu} v_{\mu} \bar{H}\right\rangle+$ H.c. & & 161 \\
\hline$\left\langle H\left\langle\nabla^{\mu} f_{+\mu}{ }^{\nu} u^{\lambda}\right\rangle \gamma_{5} \gamma_{\nu} v_{\lambda} \bar{H}\right\rangle$ & 19 & 53 & $\left\langle H u^{\mu} \chi_{-} u^{\nu} \gamma_{5} \gamma_{\mu} v_{\nu} \bar{H}\right\rangle+$ H.c. & 102 & 162 \\
\hline$\left\langle H\left\langle\nabla^{\mu} f_{+\mu}{ }^{\nu} u^{\lambda}\right\rangle \gamma_{5} \gamma_{\lambda} v_{\nu} \bar{H}\right\rangle$ & 20 & 54 & $\left\langle H\left\langle u^{\mu} u^{\nu} \chi_{-}\right\rangle \gamma_{5} \gamma_{\mu} v_{\nu} \bar{H}\right\rangle+$ H.c. & & 163 \\
\hline
\end{tabular}


TABLE X. (Continued)

\begin{tabular}{|c|c|c|c|c|c|}
\hline$P_{n}$ & $S U(2)$ & $S U(3)$ & $P_{n}$ & $S U(2)$ & $S U(3)$ \\
\hline$\left\langle H\left\langle\nabla^{\mu} f_{+}{ }^{\nu \lambda} u_{\mu}\right\rangle \gamma_{5} \gamma_{\nu} v_{\lambda} \bar{H}\right\rangle$ & 21 & 55 & $\left\langle H\left\langle\chi_{-}\right\rangle u^{\mu} u^{\nu} \gamma_{5} \gamma_{\mu} v_{\nu} \bar{H}\right\rangle+$ H.c. & & 164 \\
\hline$\left\langle H\left\langle\nabla^{\mu} f_{+}{ }^{\nu \lambda} u_{\nu}\right\rangle \gamma_{5} \gamma_{\mu} v_{\lambda} \bar{H}\right\rangle$ & 22 & 56 & $i\left\langle H f_{+}{ }^{\mu \nu} \chi_{-} \gamma_{5} \gamma_{\mu} v_{\nu} \bar{H}\right\rangle+$ H.c. & 103 & 165 \\
\hline$\left\langle H\left\langle\nabla^{\mu} f_{+}{ }^{\nu \lambda} u^{\rho}\right\rangle \gamma_{5} \gamma_{\nu} v_{\mu} v_{\lambda} v_{\rho} \bar{H}\right\rangle$ & 23 & 57 & $i\left\langle H\left\langle f_{+}{ }^{\mu \nu}\right\rangle \chi_{-} \gamma_{5} \gamma_{\mu} v_{\nu} \bar{H}\right\rangle$ & 104 & \\
\hline$\left\langle H\left\langle f_{+}{ }^{\mu \nu} f_{-\mu}{ }^{\lambda}\right\rangle \gamma_{5} \gamma_{\nu} v_{\lambda} \bar{H}\right\rangle$ & 24 & & $i\left\langle H\left\langle f_{+}{ }^{\mu \nu} \chi_{-}\right\rangle \gamma_{5} \gamma_{\mu} v_{\nu} \bar{H}\right\rangle$ & 105 & 166 \\
\hline$\left\langle H\left\langle f_{+}{ }^{\mu \nu} h_{\mu}{ }^{\lambda}\right\rangle \gamma_{5} \gamma_{\nu} v_{\lambda} \bar{H}\right\rangle$ & 25 & & $i\left\langle H\left\langle\chi_{-}\right\rangle f_{+}{ }^{\mu \nu} \gamma_{5} \gamma_{\mu} v_{\nu} \bar{H}\right\rangle$ & 106 & 167 \\
\hline$\left\langle H\left\langle f_{+}{ }^{\mu \nu} h_{\mu}{ }^{\lambda}\right\rangle \gamma_{5} \gamma_{\lambda} v_{\nu} \bar{H}\right\rangle$ & 26 & & $\left\langle H \chi_{-}^{2} \bar{H}\right\rangle$ & 107 & 168 \\
\hline$\left\langle H\left\langle f_{+}{ }^{\mu \nu} h^{\lambda \rho}\right\rangle \gamma_{5} \gamma_{\mu} v_{\nu} v_{\lambda} v_{\rho} \bar{H}\right\rangle$ & 27 & & $\left\langle H\left\langle\chi_{-}\right\rangle \chi_{-} \bar{H}\right\rangle$ & 108 & 169 \\
\hline$i \varepsilon^{\mu \nu \lambda \rho}\left\langle H f_{+\mu \nu} f_{-\lambda \rho} \bar{H}\right\rangle+$ H.c. & 28 & 58 & $\left\langle H\left\langle\chi_{-}\right\rangle\left\langle\chi_{-}\right\rangle \bar{H}\right\rangle$ & & 170 \\
\hline$i \varepsilon^{\mu \nu \lambda \rho}\left\langle H f_{+\mu \nu} f_{-\lambda}^{\sigma} v_{\rho} v_{\sigma} \bar{H}\right\rangle+$ H.c. & 29 & 59 & $i\left\langle H\left\langle u^{\mu} u^{\nu} f_{-\mu}{ }^{\lambda}\right\rangle \gamma_{5} \gamma_{\nu} v_{\lambda} \bar{H}\right\rangle+$ H.c. & 109 & 171 \\
\hline$i \varepsilon^{\mu \nu \lambda \rho}\left\langle H f_{+\mu \nu} h_{\lambda}{ }^{\sigma} v_{\rho} v_{\sigma} \bar{H}\right\rangle+$ H.c. & 30 & 60 & $i\left\langle H\left\langle u^{\mu} u^{\nu} f_{-\mu}{ }^{\lambda}\right\rangle \gamma_{5} \gamma_{\lambda} v_{\nu} \bar{H}\right\rangle+$ H.c. & 110 & 172 \\
\hline$i \varepsilon^{\mu \nu \lambda \rho}\left\langle H \nabla_{\mu} f_{+\nu}{ }^{\sigma} u_{\lambda} v_{\rho} v_{\sigma} \bar{H}\right\rangle+$ H.c. & 31 & 61 & $i\left\langle H\left\langle u^{\mu} u^{\nu} h_{\mu}{ }^{\lambda}\right\rangle \gamma_{5} \gamma_{\nu} v_{\lambda} \bar{H}\right\rangle+$ H.c. & 111 & 173 \\
\hline$i\left\langle H f_{-}{ }^{\mu \nu} \chi_{+} \gamma_{5} \gamma_{\mu} v_{\nu} \bar{H}\right\rangle+$ H.c. & 32 & 62 & $i\left\langle H\left\langle u^{\mu} u^{\nu} h_{\mu}^{\lambda}\right\rangle \gamma_{5} \gamma_{\lambda} v_{\nu} \bar{H}\right\rangle+$ H.c. & 112 & 174 \\
\hline$i\left\langle H h^{\mu \nu} \chi_{+} \gamma_{5} \gamma_{\mu} v_{\nu} \bar{H}\right\rangle+$ H.c. & 33 & 63 & $i\left\langle H\left\langle u^{\mu} u^{\nu} h^{\lambda \rho}\right\rangle \gamma_{5} \gamma_{\mu} v_{\nu} v_{\lambda} v_{\rho} \bar{H}\right\rangle+$ H.c. & 113 & 175 \\
\hline$i\left\langle H u^{\mu} \nabla^{\nu} \chi_{+} \gamma_{5} \gamma_{\mu} v_{\nu} \bar{H}\right\rangle+$ H.c. & 34 & 64 & $i\left\langle H u^{\mu} u_{\mu} f_{-}{ }^{\nu \lambda} \gamma_{5} \gamma_{\nu} v_{\lambda} \bar{H}\right\rangle+$ H.c. & & 176 \\
\hline$i\left\langle H u^{\mu} \nabla^{\nu} \chi_{+} \gamma_{5} \gamma_{\nu} v_{\mu} \bar{H}\right\rangle+$ H.c. & 35 & 65 & $i\left\langle H u^{\mu} u^{\nu} f_{-\mu}^{\lambda} \gamma_{5} \gamma_{\nu} v_{\lambda} \bar{H}\right\rangle+$ H.c. & & 177 \\
\hline$\left\langle H f_{-}{ }^{\mu \nu} \chi_{-} \sigma_{\mu \nu} \bar{H}\right\rangle+$ H.c. & 36 & 66 & $i\left\langle H u^{\mu} u^{\nu} f_{-\mu}^{\lambda} \gamma_{5} \gamma_{\lambda} v_{\nu} \bar{H}\right\rangle+$ H.c. & & 178 \\
\hline$i\left\langle H h^{\mu \nu} \chi_{-} v_{\mu} v_{\nu} \bar{H}\right\rangle+$ H.c. & 37 & 67 & $i\left\langle H u^{\mu} u^{\nu} f_{-\nu}^{\lambda} \gamma_{5} \gamma_{\mu} v_{\lambda} \bar{H}\right\rangle+$ H.c. & & 179 \\
\hline$i\left\langle H u^{\mu} \nabla_{\mu} \chi_{-} \bar{H}\right\rangle+$ H.c. & 38 & 68 & $i\left\langle H u^{\mu} u^{\nu} f_{-\nu}^{\lambda} \gamma_{5} \gamma_{\lambda} v_{\mu} \bar{H}\right\rangle+$ H.c. & & 180 \\
\hline$i\left\langle H u^{\mu} \nabla^{\nu} \chi_{-} v_{\mu} v_{\nu} \bar{H}\right\rangle+$ H.c. & 39 & 69 & $i\left\langle H u^{\mu} u^{\nu} f_{-}{ }^{\lambda \rho} \gamma_{5} \gamma_{\lambda} v_{\mu} v_{\nu} v_{\rho} \bar{H}\right\rangle+$ H.c. & & 181 \\
\hline$\left\langle H u^{\mu} \nabla^{\nu} \chi_{-} \sigma_{\mu \nu} \bar{H}\right\rangle+$ H.c. & 40 & 70 & $i\left\langle H u^{\mu} u_{\mu} h^{\nu \lambda} \gamma_{5} \gamma_{\nu} v_{\lambda} \bar{H}\right\rangle+$ H.c. & & 182 \\
\hline$i\left\langle H\left\langle h^{\mu \nu} \chi_{-}\right\rangle v_{\mu} v_{\nu} \bar{H}\right\rangle$ & 41 & 71 & $i\left\langle H u^{\mu} u^{\nu} h_{\mu}^{\lambda} \gamma_{5} \gamma_{\nu} v_{\lambda} \bar{H}\right\rangle+$ H.c. & & 183 \\
\hline$i\left\langle H\left\langle\chi_{-}\right\rangle h^{\mu \nu} v_{\mu} v_{\nu} \bar{H}\right\rangle$ & & 72 & $i\left\langle H u^{\mu} u^{\nu} h_{\mu}{ }^{\lambda} \gamma_{5} \gamma_{\lambda} v_{\nu} \bar{H}\right\rangle+$ H.c. & & 184 \\
\hline$i\left\langle H\left\langle u^{\mu} \nabla_{\mu} \chi_{-}\right\rangle \bar{H}\right\rangle$ & 42 & 73 & $i\left\langle H u^{\mu} u^{\nu} h_{\nu}^{\lambda} \gamma_{5} \gamma_{\mu} v_{\lambda} \bar{H}\right\rangle+$ H.c. & & 185 \\
\hline$i\left\langle H\left\langle\nabla^{\mu} \chi_{-}\right\rangle u_{\mu} \bar{H}\right\rangle$ & & 74 & $i\left\langle H u^{\mu} u^{\nu} h_{\nu}^{\lambda} \gamma_{5} \gamma_{\lambda} v_{\mu} \bar{H}\right\rangle+$ H.c. & & 186 \\
\hline$i\left\langle H\left\langle u^{\mu} \nabla^{\nu} \chi_{-}\right\rangle v_{\mu} v_{\nu} \bar{H}\right\rangle$ & 43 & 75 & $i\left\langle H u^{\mu} u^{\nu} h^{\lambda \rho} \gamma_{5} \gamma_{\mu} v_{\nu} v_{\lambda} v_{\rho} \bar{H}\right\rangle+$ H.c. & & 187 \\
\hline$i\left\langle H\left\langle\nabla^{\mu} \chi_{-}\right\rangle u^{\nu} v_{\mu} v_{\nu} \bar{H}\right\rangle$ & & 76 & $i\left\langle H u^{\mu} u^{\nu} h^{\lambda \rho} \gamma_{5} \gamma_{\nu} v_{\mu} v_{\lambda} v_{\rho} \bar{H}\right\rangle+$ H.c. & & 188 \\
\hline$\left\langle H\left\langle u^{\mu} \nabla^{\nu} f_{-\mu \nu}\right\rangle \bar{H}\right\rangle$ & 44 & 77 & $i\left\langle H u^{\mu} u^{\nu} h^{\lambda \rho} \gamma_{5} \gamma_{\lambda} v_{\mu} v_{\nu} v_{\rho} \bar{H}\right\rangle+$ H.c. & & 189 \\
\hline$\left\langle H\left\langle u^{\mu} \nabla^{\nu} f_{-\mu}^{\lambda}\right\rangle v_{\nu} v_{\lambda} \bar{H}\right\rangle$ & 45 & 78 & $i\left\langle H u^{\mu} f_{-\mu}^{\nu} u^{\lambda} \gamma_{5} \gamma_{\nu} v_{\lambda} \bar{H}\right\rangle+$ H.c. & & 190 \\
\hline$\left\langle H\left\langle u^{\mu} \nabla^{\nu} f_{-\nu}^{\lambda}\right\rangle v_{\mu} v_{\lambda} \bar{H}\right\rangle$ & 46 & 79 & $i\left\langle H u^{\mu} f_{-\mu}^{\nu} u^{\lambda} \gamma_{5} \gamma_{\lambda} v_{\nu} \bar{H}\right\rangle+$ H.c. & & 191 \\
\hline$\left\langle H\left\langle u^{\mu} \nabla_{\mu} h^{\nu \lambda}\right\rangle v_{\nu} v_{\lambda} \bar{H}\right\rangle$ & 47 & 80 & $i\left\langle H u^{\mu} h_{\mu}{ }^{\nu} u^{\lambda} \gamma_{5} \gamma_{\nu} v_{\lambda} \bar{H}\right\rangle+$ H.c. & & 192 \\
\hline$\left\langle H\left\langle u^{\mu} \nabla^{\nu} h^{\lambda \rho}\right\rangle v_{\mu} v_{\nu} v_{\lambda} v_{\rho} \bar{H}\right\rangle$ & 48 & 81 & $i\left\langle H u^{\mu} h_{\mu}{ }^{\nu} u^{\lambda} \gamma_{5} \gamma_{\lambda} v_{\nu} \bar{H}\right\rangle+$ H.c. & & 193 \\
\hline$\left\langle H\left\langle f_{-}{ }^{\mu \nu} h_{\mu}{ }^{\lambda}\right\rangle v_{\nu} v_{\lambda} \bar{H}\right\rangle$ & 49 & 82 & $i\left\langle H u^{\mu} h^{\nu \lambda} u^{\rho} \gamma_{5} \gamma_{\mu} v_{\nu} v_{\lambda} v_{\rho} \bar{H}\right\rangle+$ H.c. & & 194 \\
\hline$\left\langle H\left\langle h^{\mu \nu} h_{\mu \nu}\right\rangle \bar{H}\right\rangle$ & 50 & 83 & $\varepsilon^{\mu \nu \lambda \rho}\left\langle H u_{\mu} u_{\nu} f_{-\lambda \rho} \bar{H}\right\rangle+$ H.c. & 114 & 195 \\
\hline$\left\langle H\left\langle h^{\mu \nu} h_{\mu}{ }^{\lambda}\right\rangle v_{\nu} v_{\lambda} \bar{H}\right\rangle$ & 51 & 84 & $\varepsilon^{\mu \nu \lambda \rho}\left\langle H u_{\mu} u_{\nu} f_{-\lambda}^{\sigma} v_{\rho} v_{\sigma} \bar{H}\right\rangle+$ H.c. & 115 & 196 \\
\hline$\left\langle H\left\langle h^{\mu \nu} h^{\lambda \rho}\right\rangle v_{\mu} v_{\nu} v_{\lambda} v_{\rho} \bar{H}\right\rangle$ & 52 & 85 & $\varepsilon^{\mu \nu \lambda \rho}\left\langle H u_{\mu} u^{\sigma} f_{-\nu \lambda} v_{\rho} v_{\sigma} \bar{H}\right\rangle+$ H.c. & 116 & 197 \\
\hline$\left\langle H u^{\mu} \nabla^{\nu} f_{-\mu \nu} \bar{H}\right\rangle+$ H.c. & & 86 & $\varepsilon^{\mu \nu \lambda \rho}\left\langle H u_{\mu} f_{-\nu \lambda} u^{\sigma} v_{\rho} v_{\sigma} \bar{H}\right\rangle+$ H.c. & 117 & 198 \\
\hline$\left\langle H u^{\mu} \nabla^{\nu} f_{-\mu}^{\lambda} v_{\nu} v_{\lambda} \bar{H}\right\rangle+$ H.c. & & 87 & $\varepsilon^{\mu \nu \lambda \rho}\left\langle H u_{\mu} u_{\nu} h_{\lambda}{ }^{\sigma} v_{\rho} v_{\sigma} \bar{H}\right\rangle+$ H.c. & 118 & 199 \\
\hline$\left\langle H u^{\mu} \nabla^{\nu} f_{-\nu}^{\lambda} v_{\mu} v_{\lambda} \bar{H}\right\rangle+$ H.c. & & 88 & $\left\langle H\left\langle\nabla^{\mu} f_{+\mu}{ }^{\nu}\right\rangle u^{\lambda} \gamma_{5} \gamma_{\nu} v_{\lambda} \bar{H}\right\rangle$ & 119 & \\
\hline$\left\langle H u^{\mu} \nabla_{\mu} h^{\nu \lambda} v_{\nu} v_{\lambda} \bar{H}\right\rangle+$ H.c. & & 89 & $\left\langle H\left\langle\nabla^{\mu} f_{+\mu}{ }^{\nu}\right\rangle u^{\lambda} \gamma_{5} \gamma_{\lambda} v_{\nu} \bar{H}\right\rangle$ & 120 & \\
\hline$\left\langle H u^{\mu} \nabla^{\nu} h^{\lambda \rho} v_{\mu} v_{\nu} v_{\lambda} v_{\rho} \bar{H}\right\rangle+$ H.c. & & 90 & $\left\langle H\left\langle\nabla^{\mu} f_{+}{ }^{\nu \lambda}\right\rangle u_{\mu} \gamma_{5} \gamma_{\nu} v_{\lambda} \bar{H}\right\rangle$ & 121 & \\
\hline$\left\langle H f_{-}{ }^{\mu \nu} f_{-\mu \nu} \bar{H}\right\rangle$ & & 91 & $\left\langle H\left\langle\nabla^{\mu} f_{+}{ }^{\nu \lambda}\right\rangle u_{\nu} \gamma_{5} \gamma_{\mu} v_{\lambda} \bar{H}\right\rangle$ & 122 & \\
\hline$\left\langle H f_{-}{ }^{\mu \nu} f_{-\mu}{ }^{\lambda} v_{\nu} v_{\lambda} \bar{H}\right\rangle$ & & 92 & $\left\langle H\left\langle\nabla^{\mu} f_{+}{ }^{\nu \lambda}\right\rangle u^{\rho} \gamma_{5} \gamma_{\nu} v_{\mu} v_{\lambda} v_{\rho} \bar{H}\right\rangle$ & 123 & \\
\hline$\left\langle H f_{-}{ }^{\mu \nu} h_{\mu}{ }^{\lambda} v_{\nu} v_{\lambda} \bar{H}\right\rangle+$ H.c. & & 93 & $\left\langle H\left\langle f_{+}{ }^{\mu \nu}\right\rangle f_{-\mu}^{\lambda} \gamma_{5} \gamma_{\nu} v_{\lambda} \bar{H}\right\rangle$ & 124 & \\
\hline$\left\langle H h^{\mu \nu} h_{\mu \nu} \bar{H}\right\rangle$ & & 94 & $\left\langle H\left\langle f_{+}{ }^{\mu \nu}\right\rangle f_{-\mu}^{\lambda} \gamma_{5} \gamma_{\lambda} v_{\nu} \bar{H}\right\rangle$ & 125 & \\
\hline$\left\langle H h^{\mu \nu} h_{\mu}{ }^{\lambda} v_{\nu} v_{\lambda} \bar{H}\right\rangle$ & & 95 & $\left\langle H\left\langle f_{+}{ }^{\mu \nu}\right\rangle h_{\mu}{ }^{\lambda} \gamma_{5} \gamma_{\nu} v_{\lambda} \bar{H}\right\rangle$ & 126 & \\
\hline$\left\langle H h^{\mu \nu} h^{\lambda \rho} v_{\mu} v_{\nu} v_{\lambda} v_{\rho} \bar{H}\right\rangle$ & & 96 & $\left\langle H\left\langle f_{+}{ }^{\mu \nu}\right\rangle h_{\mu}^{\lambda} \gamma_{5} \gamma_{\lambda} v_{\nu} \bar{H}\right\rangle$ & 127 & \\
\hline$i\left\langle H u^{\mu} \nabla_{\mu} f_{-}{ }^{\nu \lambda} \sigma_{\nu \lambda} \bar{H}\right\rangle+$ H.c. & 53 & 97 & $\varepsilon^{\mu \nu \lambda \rho}\left\langle H\left\langle u_{\mu} f_{-\nu \lambda}\right\rangle u_{\rho} \bar{H}\right\rangle$ & & 200 \\
\hline$i\left\langle H u^{\mu} \nabla^{\nu} f_{-\nu}^{\lambda} \sigma_{\mu \lambda} \bar{H}\right\rangle+$ H.c. & 54 & 98 & $\varepsilon^{\mu \nu \lambda \rho}\left\langle H\left\langle u_{\mu} f_{-\nu}{ }^{\sigma}\right\rangle u_{\lambda} v_{\rho} v_{\sigma} \bar{H}\right\rangle$ & & 201 \\
\hline$i\left\langle H u^{\mu} \nabla^{\nu} f_{-}^{\lambda \rho} \sigma_{\mu \lambda} v_{\nu} v_{\rho} \bar{H}\right\rangle+$ H.c. & 55 & 99 & $\varepsilon^{\mu \nu \lambda \rho}\left\langle H\left\langle u_{\mu} u^{\sigma}\right\rangle f_{-\nu \lambda} v_{\rho} v_{\sigma} \bar{H}\right\rangle$ & & 202 \\
\hline
\end{tabular}


TABLE X. (Continued)

\begin{tabular}{|c|c|c|c|c|c|}
\hline$P_{n}$ & $S U(2)$ & $S U(3)$ & $P_{n}$ & $S U(2)$ & $S U(3)$ \\
\hline$i\left\langle H u^{\mu} \nabla^{\nu} f_{-}^{\lambda \rho} \sigma_{\nu \lambda} v_{\mu} v_{\rho} \bar{H}\right\rangle+$ H.c. & 56 & 100 & $\varepsilon^{\mu \nu \lambda \rho}\left\langle H\left\langle u_{\mu} u^{\sigma} f_{-\nu \lambda}\right\rangle v_{\rho} v_{\sigma} \bar{H}\right\rangle+$ H.c. & & 203 \\
\hline$i\left\langle H u^{\mu} \nabla^{\nu} h^{\lambda \rho} \sigma_{\mu \nu} v_{\lambda} v_{\rho} \bar{H}\right\rangle+$ H.c. & 57 & 101 & $\varepsilon^{\mu \nu \lambda \rho}\left\langle H\left\langle u_{\mu} h_{\nu}{ }^{\sigma}\right\rangle u_{\lambda} v_{\rho} v_{\sigma} \bar{H}\right\rangle$ & & 204 \\
\hline$i\left\langle H f_{-}^{\mu \nu} f_{-\mu}{ }^{\lambda} \sigma_{\nu \lambda} \lambda \bar{H}\right\rangle$ & 58 & 102 & $\left\langle H f_{+}{ }^{\mu \nu} f_{-\mu}{ }^{\lambda} \gamma_{5} \gamma_{\nu} v_{\lambda} \bar{H}\right\rangle+$ H.c. & & 205 \\
\hline$i\left\langle H f_{-}^{\mu \nu} f_{-}^{\lambda \rho} \sigma_{\mu \lambda} v_{\nu} v_{\rho} \bar{H}\right\rangle$ & 59 & 103 & $\left\langle H f_{+}{ }^{\mu \nu} f_{-\mu}{ }^{\lambda} \gamma_{5} \gamma_{\lambda} v_{\nu} \bar{H}\right\rangle+$ H.c. & & 206 \\
\hline$i\left\langle f_{-}{ }^{\mu \nu} h_{\mu}{ }^{\lambda} \sigma_{\nu \lambda} \bar{H}\right\rangle+$ H.c. & 60 & 104 & $\left\langle H f_{+}{ }^{\mu \nu} h_{\mu}{ }^{\lambda} \gamma_{5} \gamma_{\nu} v_{\lambda} \bar{H}\right\rangle+$ H.c. & & 207 \\
\hline$i\left\langle H f_{-}{ }^{\mu \nu} h^{\lambda \rho} \sigma_{\mu \nu} v_{\lambda} v_{\rho} \bar{H}\right\rangle+$ H.c. & 61 & 105 & $\left\langle H f_{+}{ }^{\mu \nu} h_{\mu}{ }^{\lambda} \gamma_{5} \gamma_{\lambda} v_{\nu} \bar{H}\right\rangle+$ H.c. & & 208 \\
\hline$i\left\langle H f_{-}{ }^{\mu \nu} h^{\lambda \rho} \sigma_{\mu \lambda} v_{\nu} v_{\rho} \bar{H}\right\rangle+$ H.c. & 62 & 106 & $\left\langle H\left\langle D^{\mu} D_{\mu} F_{L}^{\nu \lambda}\right\rangle \sigma_{\nu \lambda} \bar{H}\right\rangle+$ H.c. & 128 & \\
\hline$i\left\langle H h^{\mu \nu} h_{\mu}{ }^{\lambda} \sigma_{\nu \lambda} \bar{H}\right\rangle$ & 63 & 107 & $\left\langle H\left\langle D^{\mu} D^{\nu} F_{L}^{\lambda \rho}\right\rangle \sigma_{\mu \lambda} v_{\nu} v_{\rho} \bar{H}\right\rangle+$ H.c. & 129 & \\
\hline$i\left\langle H h^{\mu \nu} h^{\lambda \rho} \sigma_{\mu \lambda} v_{\nu} v_{\rho} \bar{H}\right\rangle$ & 64 & 108 & $\left\langle H\left\langle F_{L}^{\mu \nu} F_{L \mu \nu}\right\rangle \bar{H}\right\rangle+$ H.c. & 130 & 209 \\
\hline$\left\langle H \nabla^{\mu} \nabla_{\mu} f_{+}{ }^{\nu \lambda} \sigma_{\nu \lambda} \bar{H}\right\rangle$ & 65 & 109 & $\left\langle H\left\langle F_{L}{ }^{\mu \nu} F_{L \mu}{ }^{\lambda}\right\rangle v_{\nu} v_{\lambda} \bar{H}\right\rangle+$ H.c. & 131 & 210 \\
\hline$\left\langle H \nabla^{\mu} \nabla^{\nu} f_{+}^{\lambda \rho} \sigma_{\mu \lambda} v_{\nu} v_{\rho} \bar{H}\right\rangle$ & 66 & 110 & $\left\langle H\left\langle F_{L}{ }^{\mu \nu} F_{L \mu}{ }^{\lambda}\right\rangle \gamma_{5} \gamma_{\nu} v_{\lambda} \bar{H}\right\rangle+$ H.c. & 132 & 211 \\
\hline$\left\langle H\left\langle f_{+}{ }^{\mu \nu} u_{\mu}\right\rangle u^{\lambda} \sigma_{\nu \lambda} \bar{H}\right\rangle$ & 67 & & $\left\langle H\left\langle F_{L}{ }^{\mu \nu}\right\rangle\left\langle F_{L \mu \nu}\right\rangle \bar{H}\right\rangle+$ H.c. & 133 & \\
\hline$\left\langle H\left\langle f_{+}{ }^{\mu \nu} u^{\lambda}\right\rangle u_{\mu} \sigma_{\nu \lambda} \bar{H}\right\rangle$ & 68 & & $\left\langle H\left\langle F_{L}{ }^{\mu \nu}\right\rangle\left\langle F_{L \mu}{ }^{\lambda}\right\rangle v_{\nu} v_{\lambda} \bar{H}\right\rangle+$ H.c. & 134 & \\
\hline$\left\langle H\left\langle f_{+}{ }^{\mu \nu} u^{\lambda}\right\rangle u_{\lambda} \sigma_{\mu \nu} \bar{H}\right\rangle$ & 69 & & $\left\langle H\left\langle\chi \chi^{\dagger}\right\rangle \bar{H}\right\rangle$ & 135 & 212 \\
\hline$\left\langle H\left\langle f_{+}{ }^{\mu \nu} u^{\lambda}\right\rangle u^{\rho} \sigma_{\mu \nu} v_{\lambda} v_{\rho} \bar{H}\right\rangle$ & 70 & & $\langle H \operatorname{det} \chi \bar{H}\rangle+$ H.c. & 136 & \\
\hline$\left\langle H\left\langle f_{+}{ }^{\mu \nu} u^{\lambda}\right\rangle u^{\rho} \sigma_{\mu \lambda} v_{\nu} v_{\rho} \bar{H}\right\rangle$ & 71 & & & & \\
\hline
\end{tabular}

The long relations in the seventh column in Table VII are

$$
\begin{aligned}
& \tilde{C}_{50}^{(3)}=\tilde{C}_{52}^{(3)}=\frac{1}{2} \tilde{C}_{1}^{(3)}+\tilde{C}_{10}^{(3)}-\tilde{C}_{13}^{(3)}+\tilde{C}_{17}^{(3)}-\tilde{C}_{19}^{(3)}, \quad \tilde{C}_{51}^{(3)}=-\tilde{C}_{1}^{(3)}-2 \tilde{C}_{10}^{(3)}+\tilde{C}_{17}^{(3)}-\tilde{C}_{19}^{(3)}, \\
& \tilde{C}_{53}^{(3)}=\tilde{C}_{55}^{(3)}=\frac{1}{2} \tilde{C}_{1}^{(3)}+\tilde{C}_{10}^{(3)}+\tilde{C}_{11}^{(3)}-\tilde{C}_{15}^{(3)}+\tilde{C}_{17}^{(3)}+\tilde{C}_{18}^{(3)}-\tilde{C}_{20}^{(3)}-\frac{1}{2} \tilde{C}_{35}^{(3)}, \\
& \tilde{C}_{54}^{(3)}=-\tilde{C}_{1}^{(3)}-2 \tilde{C}_{10}^{(3)}-2 \tilde{C}_{11}^{(3)}+\tilde{C}_{17}^{(3)}+\tilde{C}_{18}^{(3)}-\tilde{C}_{20}^{(3)}+\tilde{C}_{35}^{(3)}, \quad \tilde{C}_{58}^{(3)}=-\frac{3}{2} \tilde{C}_{17}^{(3)}-\frac{3}{2} \tilde{C}_{18}^{(3)}+\frac{1}{2} \tilde{C}_{20}^{(3)}, \\
& \tilde{C}_{59}^{(3)}=-\tilde{C}_{17}^{(3)}-\tilde{C}_{18}^{(3)}+\tilde{C}_{20}^{(3)}, \quad \tilde{C}_{70}^{(3)}=\tilde{C}_{33}^{(3)}+\tilde{C}_{34}^{(3)}-\tilde{C}_{35}^{(3)} .
\end{aligned}
$$

[1] S. Weinberg, Phenomenological Lagrangians, Physica (Amsterdam) 96A, 327 (1979).

[2] J. Gasser and H. Leutwyler, Chiral perturbation theory to one loop, Ann. Phys. (N.Y.) 158, 142 (1984).

[3] J. Gasser and H. Leutwyler, Chiral perturbation theory: Expansions in the mass of the strange quark, Nucl. Phys. B250, 465 (1985).

[4] A. Krause, Baryon matrix elements of the vector current in chiral perturbation theory, Helv. Phys. Acta 63, 3 (1990).

[5] E. Jenkins and A. V. Manohar, Chiral corrections to the baryon axial currents, Phys. Lett. B 259, 353 (1991).

[6] T. R. Hemmert, B. R. Holstein, and J. Kambor, Heavy baryon chiral perturbation theory with light deltas, J. Phys. G 24, 1831 (1998).

[7] T.-M. Yan, H.-Y. Cheng, C.-Y. Cheung, G.-L. Lin, Y. C. Lin, and H.-L. Yu, Heavy quark symmetry and chiral dynamics, Phys. Rev. D 46, 1148 (1992).
[8] M. B. Wise, Chiral perturbation theory for hadrons containing a heavy quark, Phys. Rev. D 45, R2188 (1992).

[9] N. Isgur and M. B. Wise, Weak decays of heavy mesons in the static quark approximation, Phys. Lett. B 232, 113 (1989).

[10] N. Isgur and M. B. Wise, Weak transition form factors between heavy mesons, Phys. Lett. B 237, 527 (1990).

[11] H. Georgi, An effective field theory for heavy quarks at lowenergies, Phys. Lett. B 240, 447 (1990).

[12] G. Burdman and J. F. Donoghue, Union of chiral and heavy quark symmetries, Phys. Lett. B 280, 287 (1992).

[13] F.-K. Guo, C. Hanhart, S. Krewald, and U.-G. Meißner, Subleading contributions to the width of the $D_{s 0}^{*}(2317)$, Phys. Lett. B 666, 251 (2008).

[14] F.-K. Guo, C. Hanhart, and U.-G. Meißner, Interactions between heavy mesons and Goldstone bosons from chiral dynamics, Eur. Phys. J. A 40, 171 (2009). 
[15] M. Altenbuchinger, L. S. Geng, and W. Weise, Scattering lengths of Nambu-Goldstone bosons off $D$ mesons and dynamically generated heavy-light mesons, Phys. Rev. D 89, 014026 (2014).

[16] Z.-W. Liu, N. Li, and S.-L. Zhu, Chiral perturbation theory and the $\bar{B} \bar{B}$ strong interaction, Phys. Rev. D 89, 074015 (2014).

[17] D.-L. Yao, M.-L. Du, F.-K. Guo, and U.-G. Meißner, Oneloop analysis of the interactions between charmed mesons and Goldstone bosons, J. High Energy Phys. 11 (2015) 058.

[18] Z.-H. Guo, U.-G. Meißner, and D.-L. Yao, New insights into the $D_{s 0}^{*}(2317)$ and other charm scalar mesons, Phys. Rev. D 92, 094008 (2015).

[19] Z.-G. Wang, $D_{s 3}^{*}(2860)$ and $D_{s 1}^{*}(2860)$ as the $1 \mathrm{D} c \bar{s}$ states, Eur. Phys. J. C 75, 25 (2015).

[20] M.-L. Du, F.-K. Guo, U.-G. Meißner, and D.-L. Yao, Aspects of the low-energy constants in the chiral Lagrangian for charmed mesons, Phys. Rev. D 94, 094037 (2016).

[21] B.-X. Sun, F.-Y. Dong, and J.-L. Pang, Study of X(5568) in a unitary coupled-channel approximation of $B \bar{K}$ and $B_{s} \pi$, Chin. Phys. C 41, 074104 (2017).

[22] H. Xu, B. Wang, Z.-W. Liu, and X. Liu, $D D^{*}$ potentials in chiral perturbation theory and possible molecular states, Phys. Rev. D 99, 014027 (2019).

[23] H.-Y. Cheng and F.-S. Yu, Masses of scalar and axial-vector B mesons revisited, Eur. Phys. J. C 77, 668 (2017).

[24] M.-L. Du, F.-K. Guo, U.-G. Meißner, and D.-L. Yao, Study of open-charm $0^{+}$states in unitarized chiral effective theory with one-loop potentials, Eur. Phys. J. C 77, 728 (2017).

[25] X.-Y. Guo, Y. Heo, and M. F. M. Lutz, On chiral extrapolations of charmed meson masses and coupled-channel reaction dynamics, Phys. Rev. D 98, 014510 (2018).

[26] H.-X. Chen, W. Chen, X. Liu, Y.-R. Liu, and S.-L. Zhu, A review of the open charm and open bottom systems, Rep. Prog. Phys. 80, 076201 (2017).

[27] H. W. Fearing and S. Scherer, Extension of the chiral perturbation theory meson Lagrangian to order $p^{6}$, Phys. Rev. D 53, 315 (1996).

[28] P. Herrera-Siklódy, J. I. Latorre, P. Pascual, and J. Taron, Chiral effective Lagrangian in the large $N_{c}$ limit: The nonet case, Nucl. Phys. B497, 345 (1997).

[29] J. Bijnens, G. Colangelo, and G. Ecker, The mesonic chiral Lagrangian of order $p^{6}$, J. High Energy Phys. 02 (1999) 020 .

[30] J. Bijnens, L. Girlanda, and P. Talavera, The anomalous chiral Lagrangian of order $p^{6}$, Eur. Phys. J. C 23, 539 (2002).

[31] T. Ebertshäuser, H. W. Fearing, and S. Scherer, The anomalous chiral perturbation theory meson Lagrangian to order $p^{6}$ reexamined, Phys. Rev. D 65, 054033 (2002).

[32] O. Catà and V. Mateu, Chiral perturbation theory with tensor sources, J. High Energy Phys. 09 (2007) 078.
[33] C. Haefeli, M. A. Ivanov, M. Schmid, and G. Ecker, On the mesonic Lagrangian of order $p^{6}$ in chiral SU(2), arXiv:0705.0576.

[34] S.-Z. Jiang, F.-J. Ge, and Q. Wang, Full pseudoscalar mesonic chiral Lagrangian at $p^{6}$ order under the unitary group, Phys. Rev. D 89, 074048 (2014).

[35] J. Bijnens, N. Hermansson-Truedsson, and S. Wang, The order $p^{8}$ mesonic chiral Lagrangian, J. High Energy Phys. 01 (2019) 102.

[36] N. Fettes, U.-G. Meißner, M. Mojžišs, and S. Steininger, The chiral effective pion nucleon Lagrangian of order $p^{4}$, Ann. Phys. (N.Y.) 283, 273 (2000).

[37] J. A. Oller, M. Verbeni, and J. Prades, Meson-baryon effective chiral Lagrangians to $\mathcal{O}\left(q^{3}\right)$, J. High Energy Phys. 09 (2006) 079.

[38] M. Frink and U.-G. Meißner, On the chiral effective mesonbaryon Lagrangian at third order, Eur. Phys. J. A 29, 255 (2006).

[39] S.-Z. Jiang, Q.-S. Chen, and Y.-R. Liu, Meson-baryon effective chiral Lagrangians at order $p^{4}$, Phys. Rev. D 95, 014012 (2017).

[40] S.-Z. Jiang, Y.-R. Liu, and H.-Q. Wang, Chiral Lagrangian with $\Delta(1232)$ to one loop, Phys. Rev. D 97, 014002 (2018).

[41] S.-Z. Jiang, Y.-R. Liu, H.-Q. Wang, and Q.-H. Yang, Chiral Lagrangians with decuplet baryons to one loop, Phys. Rev. D 97, 054031 (2018).

[42] M. Holmberg and S. Leupold, The relativistic chiral Lagrangian for decuplet and octet baryons at next-to-leading order, Eur. Phys. J. A 54, 103 (2018).

[43] A. Roessl, Pion kaon scattering near the threshold in chiral SU(2) perturbation theory, Nucl. Phys. B555, 507 (1999).

[44] M.-L. Du, F.-K. Guo, and U.-G. Meißner, Subtraction of power counting breaking terms in chiral perturbation theory: spinless matter fields, J. High Energy Phys. 10 (2016) 122.

[45] M.-L. Du, F.-K. Guo, and U.-G. Meißner, One-loop renormalization of the chiral Lagrangian for spinless matter fields in the SU(N) fundamental representation, J. Phys. G 44, 014001 (2017).

[46] J. Gasser, M. E. Sainio, and A. Švarc, Nucleons with chiral loops, Nucl. Phys. B307, 779 (1988).

[47] M. B. Wise, Combining chiral and heavy quark symmetry, arXiv:hep-ph/9306277.

[48] E. E. Jenkins, A. V. Manohar, and M. B. Wise, Baryons containing a heavy quark as solitons, Nucl. Phys. B396, 27 (1993).

[49] R. Casalbuoni, A. Deandrea, N. D. Bartolomeo, R. Gatto, F. Feruglio, and G. Nardulli, Phenomenology of heavy meson chiral Lagrangians, Phys. Rep. 281, 145 (1997).

[50] H. Georgi, Heavy Quark Effective Field Theory (1992), pp. 589-630, https://www.worldscientific.com/doi/abs/ 10.1142/9789814439725_0011.

[51] A. V. Manohar and M. B. Wise, Heavy Quark Physics (Cambridge University Press, Cambridge, England, 2000), Vol. 10, pp. 1-191. 